\title{
INVESTIGATION OF PARAMETERS INFLUENCING REVERSE FAULT RUPTURE PROPAGATION TO THE GROUND SURFACE
}

\author{
A Thesis \\ presented to \\ the Faculty of California Polytechnic State University, \\ San Luis Obispo
}

\author{
In Partial Fulfillment \\ of the Requirements for the Degree \\ Master of Science in Civil and Environmental Engineering
}

by

Kevin V. Stanton

December, 2013 
(C) 2013

Kevin V. Stanton

ALL RIGHTS RESERVED 
TITLE:

AUTHOR:

DATE SUBMITTED:

COMMITTEE CHAIR:

COMMITTEE CHAIR:

COMMITTEE MEMBER:
Investigation of Parameters Influencing Reverse

Fault Rupture Propagation to the Ground Surface

Kevin Stanton

December, 2013

Robb Eric S. Moss, Ph.D., P.E.

Associate Professor of Civil and Environmental Engineering

COMMITTEE MEMBER: John Jeremy Jasbinsek, Ph.D. Assistant Professor of Physics

Gregg Fiegel, Ph.D., P.E., G.E.

Professor of Civil and Environmental Engineering 


\begin{abstract}
Investigation of Parameters Influencing Reverse Fault Rupture Propagation to the Ground Surface

Kevin V. Stanton
\end{abstract}

Surface fault rupture poses a serious threat to infrastructure in many seismically active regions, but knowledge about the factors which control the likelihood of surface displacement is limited. Current probabilistic frameworks rely only on fault mechanism and moment magnitude to predict the probability of rupture to the ground surface. However, recent work has shown that there may be other parameters which also deserve consideration. For example, statistical analyses have demonstrated that variation in near surface material stiffness may significantly affect the probability of surface rupture over reverse faults. In addition, numerical investigations indicate that the rupture history of native soil deposits also greatly influences the nature of rupture propagation. Given that evidence exists which suggests multiple variables are at work, this study aimed to improve our understanding of which are most critical for predicting surface fault rupture hazard. We sought to generate physical evidence concerning the impact of near surface soil stiffness, soil type, and rupture history on fault rupture propagation. A 3 meter long by 1 meter wide fault box apparatus was constructed to simulate idealized reverse fault rupture oriented at $45^{\circ}$ beneath $60 \mathrm{~cm}$ of soil. Relatively large dimensions were chosen so that shear wave velocity measurements could be taken directly without interference from the walls of the apparatus. Experiments were conducted on loose sand, dense sand, stiff clay, and soft clay. The same sand was used for both the dense and loose sand 
experiments and is identified as Monterey \#2/16. The clay was a scale model mixture of San Francisco Bay Mud and consisted of kaolinite, bentonite, class C fly ash, and water. Separate batches of clay were mixed with differing final water contents for the stiff and soft clay experiments. In each case, the fault box was filled to $60 \mathrm{~cm}$ and rupture was driven to the surface in two phases. The first phase represented an undisturbed native soil deposit with no existing shear band. The second simulated repeat rupture along a preexisting shear band. The results indicate that increasing material stiffness promotes rupture propagation in both sand and clay. When disturbed soil is re-ruptured, surface rupture occurred much more readily in all materials. Overall, the presence of a preexisting shear band was shown to have the greatest impact on the likelihood of surface rupture, though both material stiffness and type were also found to have a strong influence as well. The fault box experiments support the findings from previous work as well as shed new light on which parameters are most critical for accurate surface rupture predictions.

Keywords: Surface rupture, fault box, shear band, stiffness 


\section{ACKNOWLEDGMENTS}

I would like to recognize my advisor Prof. Robb Moss for his resolute guidance and patience. The proficient combination of technical and practical advice he so readily offered was essential for me to overcome the numerous hurdles I encountered throughout this research. Moreover, his enthusiastic support enabled me to take this project and make it a success, granting an exceptionally rewarding experience.

I also thank the other members of my thesis committee, Dr. Gregg Fiegel and Dr. John Jasbinsek, for taking the time to read this paper and offer their feedback. In addition, I would like to acknowledge them and all of the faculty who have instructed me over the years at Cal Poly. With all of their dedication in lectures, labs, and office hours, they ultimately prepared me for the challenges that came with this work

To everyone who helped me through the experimentation process, I am greatly appreciative. This would not have been possible without Nha Ha, Jason Auyeung, Jack Sunderland, Jay Ressler, Melanie Greenfield, Elizabeth Villanueva and many others who donated their time and strength to assist during testing.

Construction of the fault box was another task I could not have completed without aid. I am grateful to Robby Nielson and Moises Buelna who offered me their tools for nearly an entire year. I would also like to extend thanks to Xi Shen who provided me with the equipment I needed on a regular basis and was of great help moving the mixer and coordinating the delivery of the sand. 


\section{TABLE OF CONTENTS}

LIST OF TABLES .X

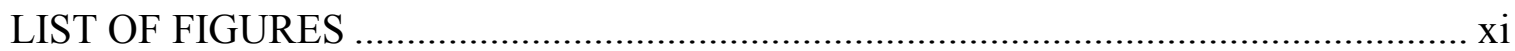

1. STATEMENT OF RESEARCH ….............................................................

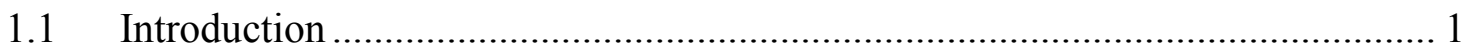

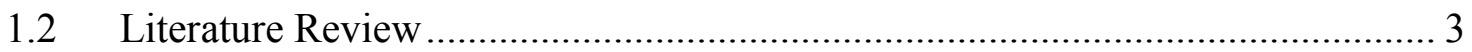

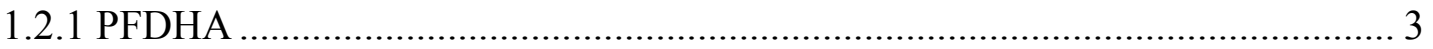

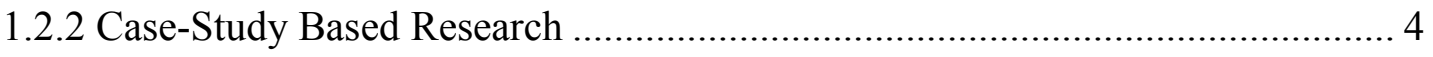

1.2.3 Previous Physical and Numerical Research ............................................... 9

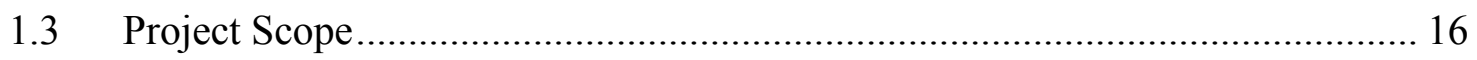

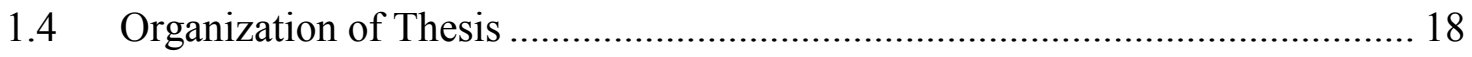

2. FAULT BOX DESIGN, CONSTRUCTION, AND INSTRUMENTATION ...........19

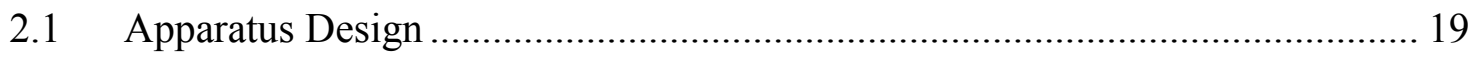

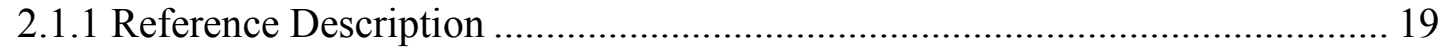

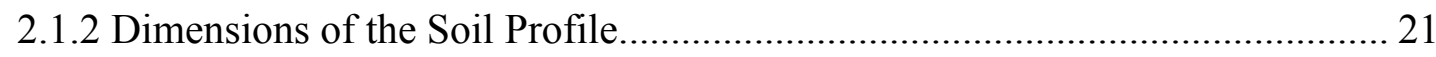

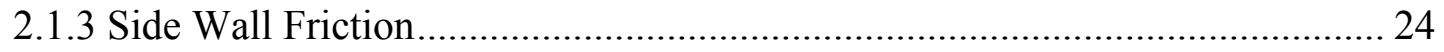

2.2 Construction Materials ........................................................................... 25

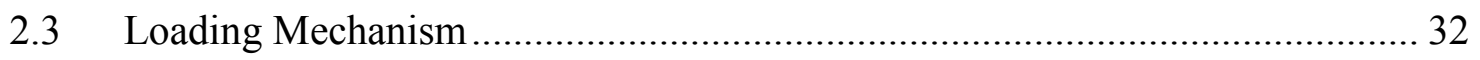

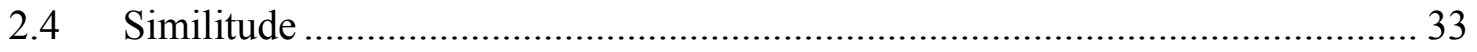


2.5 Noteworthy Complications 34

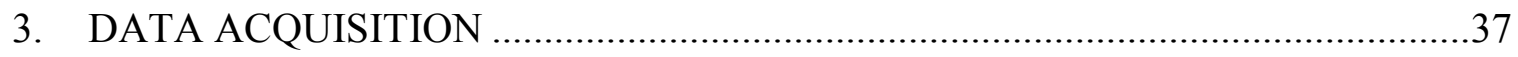

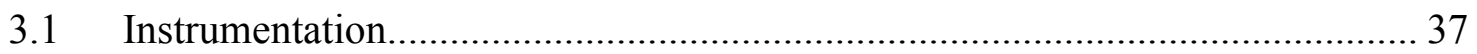

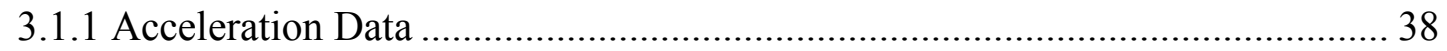

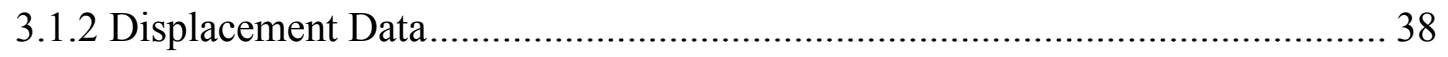

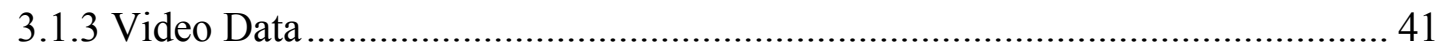

3.2 Shear Wave Velocity........................................................................... 41

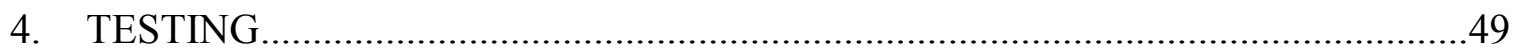

4.1 Material Properties .................................................................................... 49

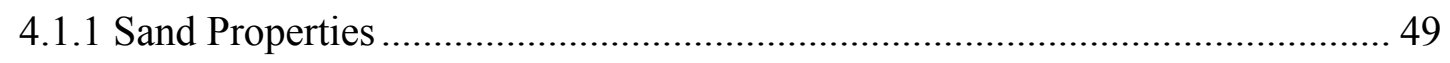

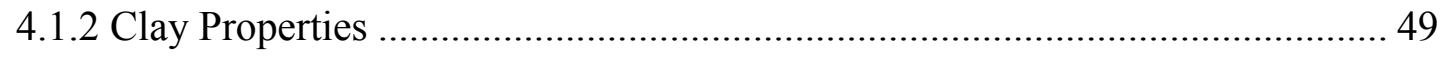

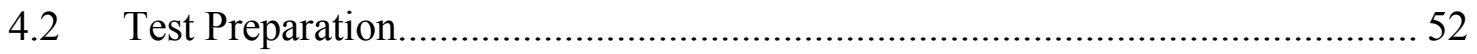

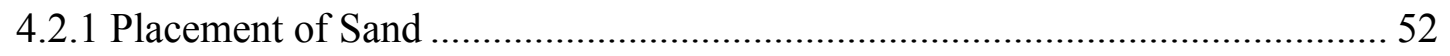

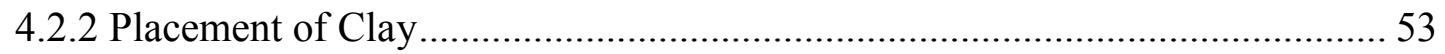

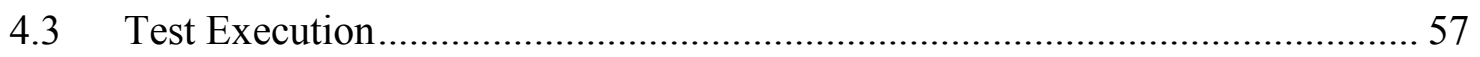

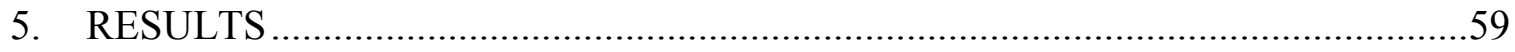

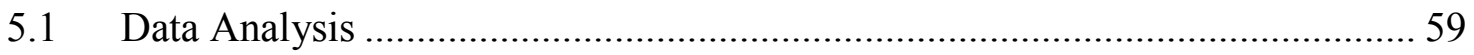

5.2 Procedure for Determining Displacement at Surface Rupture........................ 67

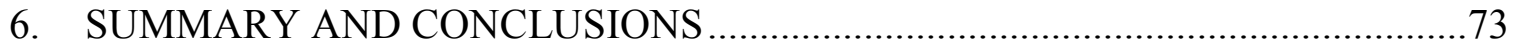

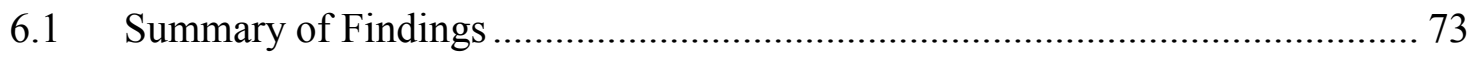

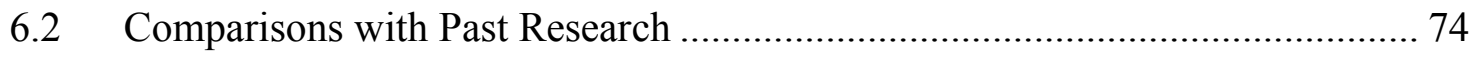

6.3 Sources of Experimental Error .......................................................... 78 
6.3.1 Defining Surface Rupture in Loose Sand

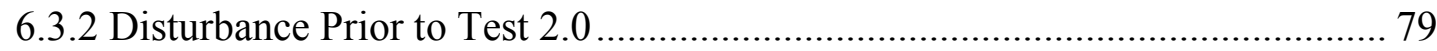

6.3.3 Effects of Marker Beds.......................................................................... 79

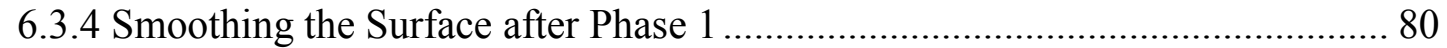

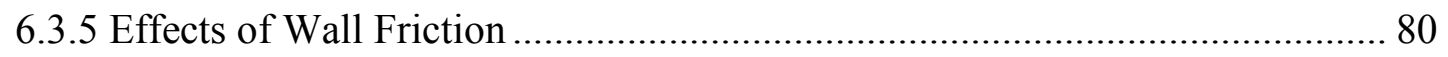

6.4 Recommendations for Future Research .............................................. 81

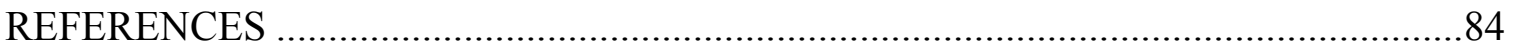




\section{LIST OF TABLES}

Table

Page

1. Sample iterations performed to estimate the stress transferred across the fault box.

2. Scale factors of engineering variables in terms of geometric scaling factor $(\lambda)$ (adapted from Iai. 1989; Meymand, 1998)

3. Dominant frequencies recorded by base (input) and top (surface) accelerometers

4. Average shear wave velocity and range of 12 hammer blow tests for each material 48

5. Recipe used to develop model mixture of San Francisco Bay Mud. 50

6. Water content data for the stiff and soft clay mixtures close to the time of testing. 51

7. Average undrained shear strengths of each layer in stiff and soft clay. 51

8. Basal displacement required for surface rupture given material type, relative stiffness, state and shear wave velocity

9. Normalized basal displacement required for surface rupture from this study and other comparable research

10. Summary of the deviations in fault emergence locations for phase 1 of each test. 


\section{LIST OF FIGURES}

Figure

Page

1. 6 meters of reverse fault surface rupture from the 1999 Chi Chi Taiwan

Earthquake. The displacement created a new waterfall and damaged the

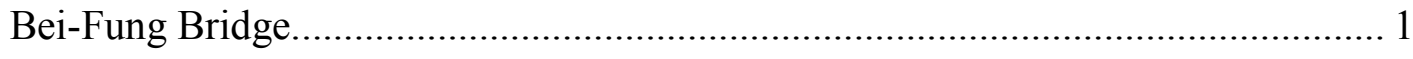

2. From Moss and Ross (2011). The probability of surface rupture for reverse, normal, and all slip types. Normal and all slip types are from Youngs et al. (2003)

3. From Moss et al. (2013). Probability of surface fault rupture as a function of thirty meters shear wave velocity bins for (a) Reverse Faulting and (b) Strike Slip Faulting. The boundary used to define stiff from soft is a thirty meter shear

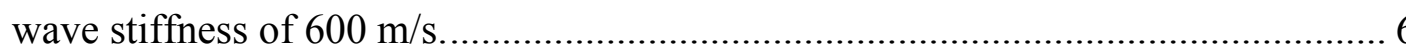

4. From Bray et al. (1994a). The Patton Bay Fault at Beach and Sea Cliff near Jeannie Point (Dotted line delineates main fault trace; Photograph by Bonilla) ....... 8

5. From Bray (1994a). Path of reverse fault rupture through soil: (a) stiff earth materials, steep dip; (b) stiff earth materials, shallow dip; (c) ductile earth materials 9

6. Finite Element prediction of normalized height of shear rupture zone as a function of clay's failure strain. From Bray et al. (1994b). 10

7. From Anastosopoulos et al. (2007). Normalized fault scarp height $s / h$ versus normalized bedrock displacement $h / H$ : (a) normal fault, $\alpha=60^{\circ}$; (b) normal fault, $\alpha=45^{\circ}$; (c) reverse fault, $\alpha=60^{\circ}$; and (d) reverse fault, $\alpha=45^{\circ}$ (dense sand: $\varphi=45^{\circ}, \psi=18^{\circ}$, and $\gamma_{\mathrm{y}}=1.5 \%$; loose sand: $\varphi=32^{\circ}, \psi=3^{\circ}$, and $\gamma_{\mathrm{y}}=3 \%$ ). 11 
8. From Oettle and Bray (2013). Comparisons between unruptured and previously ruptured soil for the same conditions as centrifuge test 28 from Bransby et al. (2008b) (reverse fault, $60^{\circ}$ dip, $15-\mathrm{m}$ soil, $2.0 \mathrm{~m}$ of previous vertical fault movement, $0.7 \mathrm{~m}$ of vertical fault movement, $\left.\mathrm{N}_{1,60}=22, \mathrm{~K}_{0}=0.45\right) \ldots 12$

9. From Bransby et al. (2008b). Free-field reverse faulting (test 28): Images of deformed soil specimen for fault throws (a) $h=0.98 \mathrm{~m}$; (b) $\mathrm{h}=1.52 \mathrm{~m}$ and (c) $\mathrm{h}=2.21 \mathrm{~m}$ 13

10. From Cole and Lade (1984). Photographs of three stages in a test on dense sand with dip angle $=60^{\circ}$ and reverse movement: (a) Undeformed condition; (b) Initiation of failure surface at bedrock fault: (c) Fully developed failure surface. 15

11. Fault box test apparatus located in the Parson's Earthquake Lab at Cal Poly, San Luis Obispo. 20

12. Simplified representation of the fault box showing only the length (horizontal) and height (vertical) dimensions. The red area is the moving section and the black area is the static section.

13. Influence value or stress under corner under uniformly loaded rectangular area. (From Holtz and Kovacs, 1981)

14. Steel cross-braces installed for bending moment resistance at hanging swivel eye double pulley connection locations. (a) Rear; (b) Front.................................... 26

15. Cable pulley system configured to lift the hanging wall of the fault box. 27

16. Floor bolts anchoring the rear end of the fault box to the floor of the Parson's Earthquake Lab. 
17. Weighting system used to anchor the front end of the fault box to the floor of

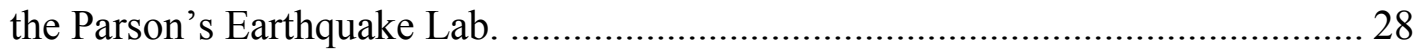

18. 1/4" soft F13 felt sheeting.. (a) Top view; (b) Side view; (c) Adhesive backing. ... 30

19. 1" thick adhesive Neoprene foam stripping. (a) Side view; (b) Top view; (c)

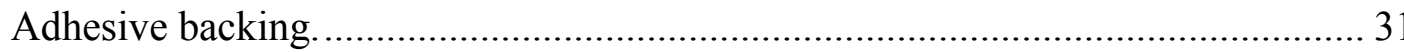

20. 3 ton ceiling crane located in the Parson's Earthquake Engineering Laboratory at Cal Poly, San Luis Obispo. 32

21. (a) Complete data acquisition setup; (b) Close up of National Instruments SCXI 1001 chassis 37

22. (a) Top accelerometer; (b) Base accelerometer; (c) Close-up of acrylic foundation and silicone protection. 38

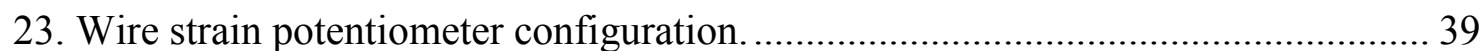

24. Displacement data collected during tests on loose sand..................................... 39

25. Displacement data collected during tests on dense sand. ................................... 40

26. Displacement data collected during tests on stiff clay..................................... 40

27. Displacement data collected during tests on soft clay. .................................... 40

28. (a) Striking location for hammer blow tests marked with white cross; (b) hammer wrapped in towel to dampen blows 42

29. Example bottom up hammer blow test waveform used for shear wave velocity determination of loose sand.

30. Example of bottom up hammer blow test waveform used for shear wave velocity determination of dense sand. 43 
31. Example of bottom up hammer blow test waveform used for shear wave velocity determination of stiff clay.

32. Example of bottom up hammer blow test waveform used for shear wave velocity determination of soft clay.

33. Acceleration time-history and discrete Fourier transform of accelerometer records in loose sand. 45

34. Acceleration time-history and discrete Fourier transform of accelerometer records in dense sand.

35. Acceleration time-history and discrete Fourier transform of accelerometer records in stiff clay.

36. Acceleration time-history and discrete Fourier transform of accelerometer records in soft clay.

37. Grain size distribution curve for the \#2/16 Monterey Sand. The solid line is the estimated average and the dashed lines are the upper and lower bounds.

38. Perforated sack used to pluviate sand. 52

39. Mixing soft clay with the ChemGrout mixer. 54

40. From Meymand (1998). Schematic of the ChemGrout mixer. 54

41. Spreading out clay from the walls inward and leveling with a painting spackle. 56

42. $2 \mathrm{~cm}$ wide elastic strips used to create marker beds in the clay. (a) Top view;

(b) Side view. 56

43. The relationship between shear wave velocity and normalized vertical displacement $(h / H)$ required for surface rupture in each material tested. 60 
44. $12.44 \mathrm{~cm}$ of basal displacement beneath loose virgin sand. The dotted line represents the rupture plane. The edges have been cropped for clarity.

45. An additional $2.19 \mathrm{~cm}$ of basal displacement beneath the loose sand with a previously existing failure plane. The dotted line represents the rupture plane. The edges have been cropped for clarity.

46. $4.01 \mathrm{~cm}$ of basal displacement beneath virgin dense sand. The dashed line represents the failure plane. The edges have been cropped for clarity. 64

47. An additional $0.50 \mathrm{~cm}$ of basal displacement beneath disturbed dense sand. The dashed line represents the failure plane. The edges have been cropped for clarity.

48. $5.53 \mathrm{~cm}$ of basal displacement beneath virgin stiff clay. The dashed line represents the failure plane. The edges have been cropped for clarity.

49. An additional $1.17 \mathrm{~cm}$ basal displacement beneath disturbed stiff clay. The dashed line represents the failure plane. The edges have been cropped for clarity.

50. $8.37 \mathrm{~cm}$ of basal displacement beneath virgin soft clay. The dashed line represents the failure plane. The edges have been cropped for clarity. 66

51. An additional $3.76 \mathrm{~cm}$ of basal displacement beneath disturbed soft clay. The dashed line represents the failure plane. The edges have been cropped for clarity. 66

52. Approximate shape of the rupture plane before it reaches the surface. The edges have been cropped for clarity. 
53. The black box is an estimate of where the rupture plane will reach the surface. The edges have been cropped for clarity.

54. Close-up view of the projected region from Figure 53 where surface rupture is likely to occur. The black line is a trance of where the sand surface and the nearest Plexiglass wall meet.

55. Examples of three successive video frames leading to surface rupture. (a) no surface rupture; (b) no surface rupture; (c) surface rupture indicated by the "kink" at which the arrow is pointing.

56. Example of the shape of the rupture plane at the moment surface rupture occurred in virgin dense sand.

57. Camera angle one.. The image is from test 2.1 (disturbed dense sand) after surface rupture had occurred.

58. Camera angle two.. The image is from test 1.1 (disturbed loose sand) after surface rupture had occurred.

59. The data from this study compared to other research. "Undefined" indicates relative stiffness was not available. 


\section{Statement of Research}

\subsection{Introduction}

Surface fault displacement hazard is a source of concern for infrastructure in seismically active regions. During earthquake events in which rupture reaches the ground surface, buildings and lifelines in the zone of deformation are at extreme risk of damage. Although surface rupture is typically mitigated by avoidance of the fault trace (Bryant, 2010), for some projects that is not possible. In such cases, a methodology known as Probabilistic Fault Displacement Hazard Analysis (PFDHA) can be employed to assess the risk (Moss and Ross, 2011; Peterson et al., 2011; Youngs et al., 2003).

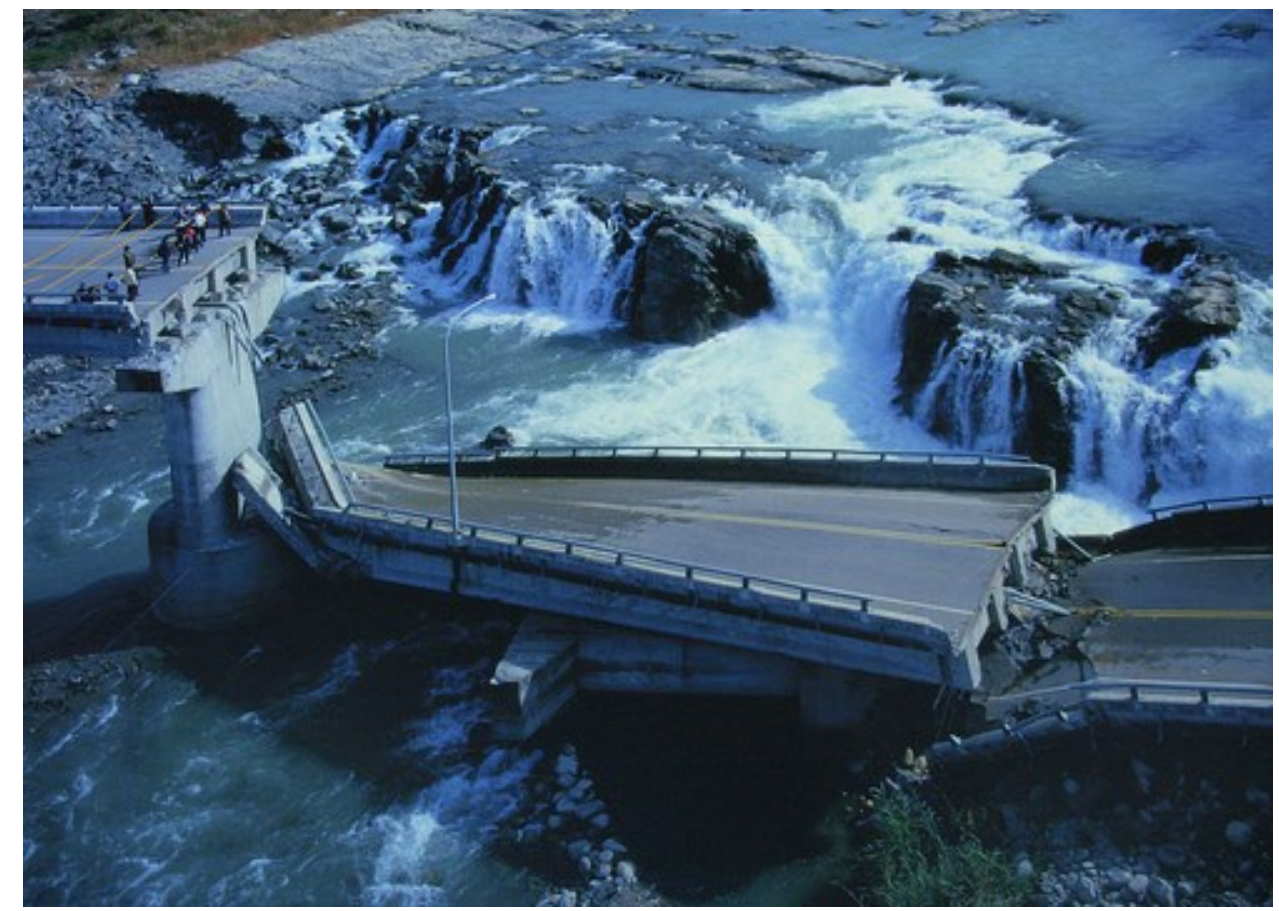

Figure 1: 6 meters of reverse fault surface rupture from the 1999 Chi Chi Taiwan Earthquake. The displacement created a new waterfall and damaged the Bei-Fung Bridge. 
Geotechnical mitigation strategies for surface rupture do exist and have successfully been implemented in the past (Oettle and Bray, 2013b). However, mitigation techniques are often expensive and not justified for smaller projects. Thus, accurate prediction of the risk of occurrence is valuable for both cost-effective design as well as life safety. Further research into the geologic factors that control rupture propagation is needed to improve the quality of hazard estimates.

There are a number of parameters which could hypothetically affect fault rupture processes. Those analyzed in this paper were chosen based on feasibility of obtaining such data through experimentation, availability of information to practitioners, and the implications from previous work. Accordingly, there are three dominant principles which shaped the goals of this work:

1) The potential for surface rupture associated with different fault mechanisms may not be qualitatively similar for all near surface geologic conditions (Moss et al., 2013).

2) Near surface material stiffness could determine whether or not reverse fault rupture reaches the ground surface (Moss et al., 2013).

3) Soil rupture history has been shown to significantly modify the fault rupture process (Oettle and Bray, 2013a).

These principles, which will be discussed in greater detail in the next section, are the basis for the experiments presented in this paper. The first directed us to focus on only one fault mechanism and the last two offer evidence pointing to the specific variables of stiffness and rupture history, suggesting that they may be essential for correctly 
predicting the probability of surface rupture for reverse mechanisms. In addition, the type of data we sought to acquire was selected so that the results of this study would be comparable with aforementioned literature.

Once a concept for an adequate set of experiments was developed, it became clear that it would be convenient to introduce another parameter, cohesion. Although prior research has involved rupture through cohesive material, there has never been a study which directly compared cohesive and cohesionless behavior. Thus, the trials were tailored to provide information about the effects of cohesion pertaining to fault rupture propagation.

\subsection{Literature Review}

\subsubsection{PFDHA}

In the form put forth in Youngs et al., (2003) for normal faulting, PFDHA produces an annual rate of events that exceed a given level of surface displacement. This involves calculating what is known as the slip exceedance, $P(D>d l m, r)$. The slip exceednace term quantifies the conditional probability of exceeding a specified amount of surface displacement given moment magnitude and that surface rupture has occurred.

$$
\begin{gathered}
P(D>d \mid m, r)=P(\text { Slip } \mid m) * P(D>d \mid m, r, \text { slip }) \\
v(d)=\alpha \int_{m_{0}}^{m_{\max }} \int_{0}^{\infty} f(m) f(r \mid m) P(D>d \mid m, r) d r d m
\end{gathered}
$$

\section{Where:}

- $D, d$ is displacement

- $m$ is moment magnitude $\left(\mathrm{M}_{\mathrm{w}}\right)$

- $r$ is distance from the main fault 
In Equation 2, the term $v(d)$ is the annual rate of events that exceed a given level of surface fault displacement. By inspection, this is dependent on the term $P($ Sliplm), which is critical for calculating the slip exceedance term. Therefore, we see that PFDHA relies heavily on accurate estimation of the probability of surface rupture, $P($ Slip $\mid m)$. Currently, this is determined using only moment magnitude as a predictor variable. Recently, following the same framework as Youngs et al., (2003), PFDHA was developed for reverse faults (Moss and Ross, 2011) and strike-slip faults (Peterson et al., 2011). The findings from Moss and Ross (2011) are shown below in Figure 2.

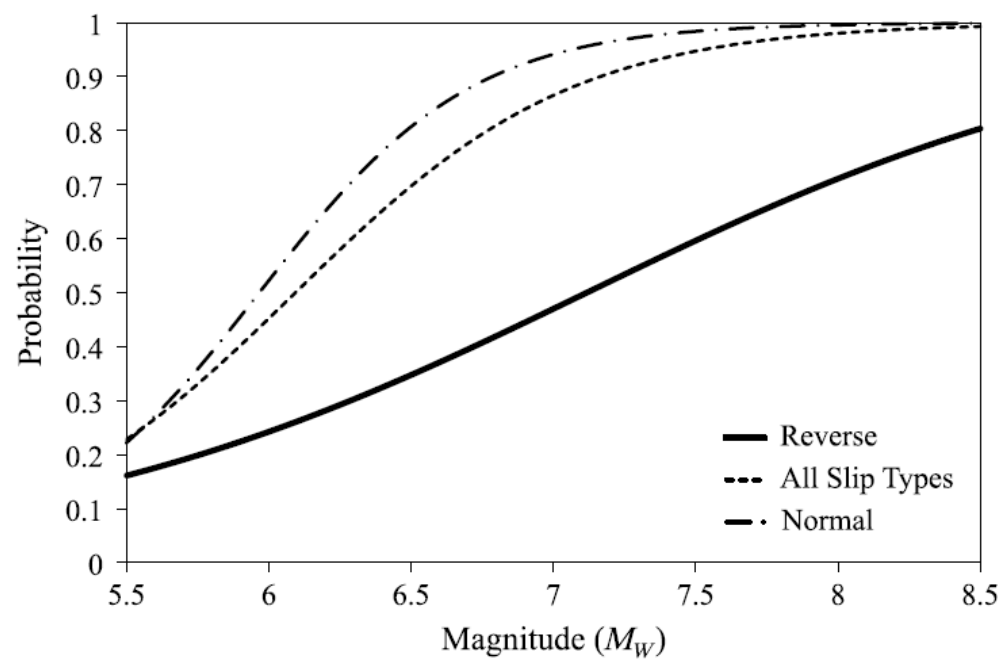

Figure 2: From Moss and Ross (2011). The probability of surface rupture for reverse, normal, and all slip types. Normal and all slip types are from Youngs et al. (2003).

\subsubsection{Case-Study Based Research}

Moss et al. (2013) suggests that material stiffness in the upper thirty meters may play a significant role in rupture propagating to the ground surface. Logistic regressions were performed on an empirical database of past earthquakes with estimates of the near surface shear wave velocity along fault traces, moment magnitude, and whether or not surface 
rupture occurred. The results indicated that reverse fault rupture is more likely to reach the surface when travelling through stiff material as opposed to soft. This may be explained by the dissimilar orientation of motion associated with reverse and strike-slip faulting. Reverse mechanisms are characterized by uplift movement which produces different loading conditions on either side of the non-seismogenic fault plane. In contrast, very similar stress states develop in the material spanning the non-seismogenic fault plane during strike-slip faulting. In response to this study, we chose to include not only material stiffness as a variable in our investigation but also determine it based on direct measurements of the characteristic shear wave velocity. This allowed us to qualitatively compare and validate/refute the findings in Moss et al., (2013). 

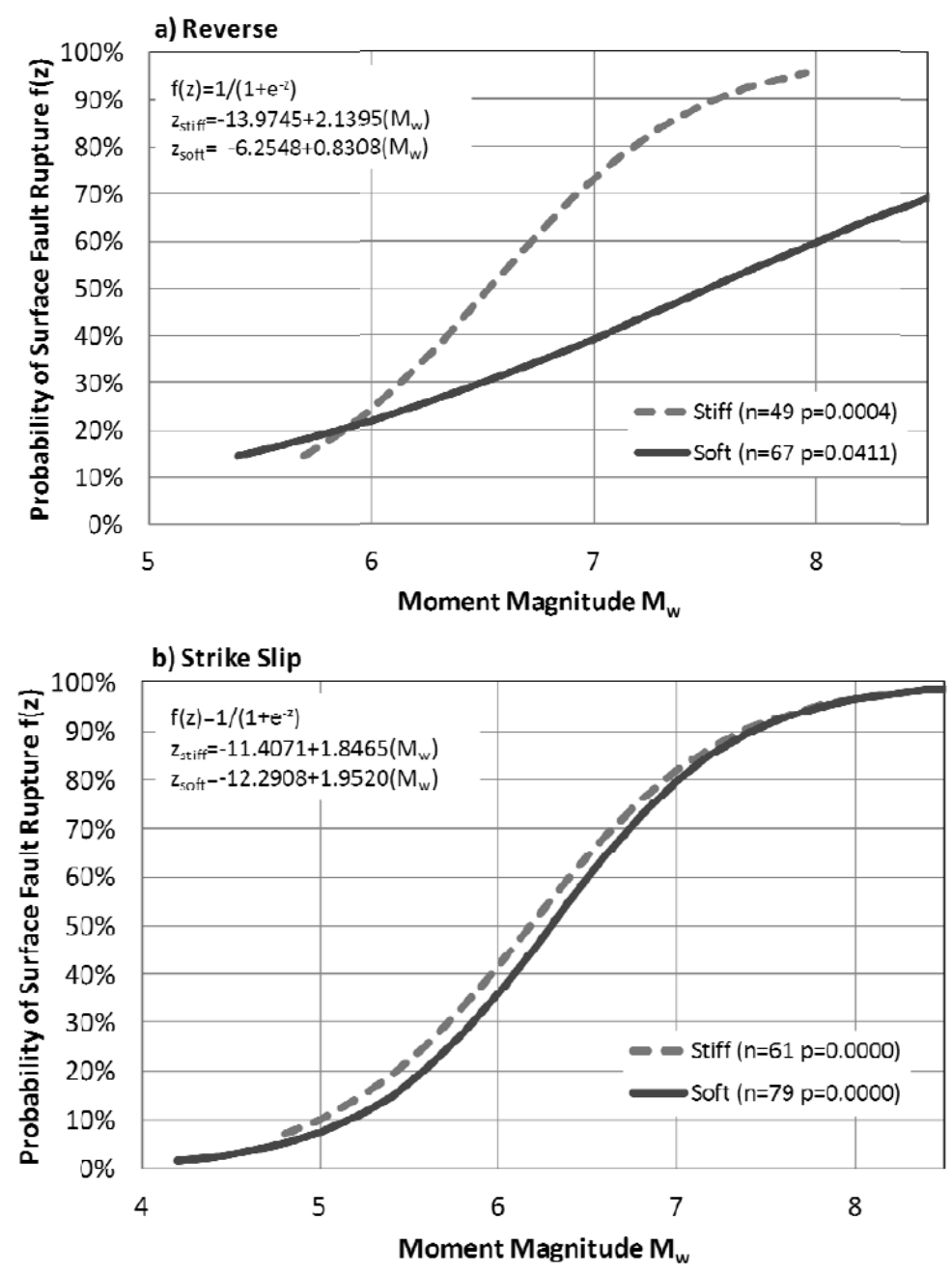

Figure 3: From Moss et al. (2013). Probability of surface fault rupture as a function of thirty meters shear wave velocity bins for (a) Reverse Faulting and (b) Strike Slip Faulting. The boundary used to define stiff from soft is a thirty meter shear wave stiffness of $600 \mathrm{~m} / \mathrm{s}$.

For events with $M_{w} \geq 6.0$, the probability of surface rupture from Figure 3 (a) for stiff material is much higher than that for reverse faulting in Figure 2. In contrast, Figure 3 (b) tells us that there exists no such difference for strike-slip faulting mechanisms. Thus, if the trends from Moss et al. (2013) are accurate, neglecting near surface stiffness in PFDHA for reverse faults beneath brittle material could result in an underestimation of the probability of surface rupture. 
Another implication from Moss et al. (2013) is that the potential for surface rupture associated with different fault mechanisms may not be qualitatively similar for all near surface geologic conditions. It was determined that near surface material stiffness has no measureable impact on the likelihood of surface rupture for strike-slip events but, as previously stated, has a significant effect for reverse events. This led us to limit the scope of this project to reverse mechanisms since material stiffness seemed less likely to delineate any existing trends related to strike-slip rupture.

Bray et al. (1994a) identifies natural evidence that overlying material can locally absorb significant movements of a reverse fault. For example, the case-study of the 1964 Alaska Earthquake on the Patton Bay Fault revealed that there was approximately 2.3 meters of vertical offset at the shore near Jeanie Point but no offset at the top of an adjacent100 meter high coastal bluff (Figure 4). In another instance, it was noted that the 1971 San Fernando earthquake $\left(\mathrm{M}_{\mathrm{w}}=6.6\right)$ resulted in surface rupture in sandstone and siltstone but not in surficial alluvium just 26 meters away. Such examples demonstrate that near surface material can play a key role in the potential for surface rupture for certain geologic conditions. 


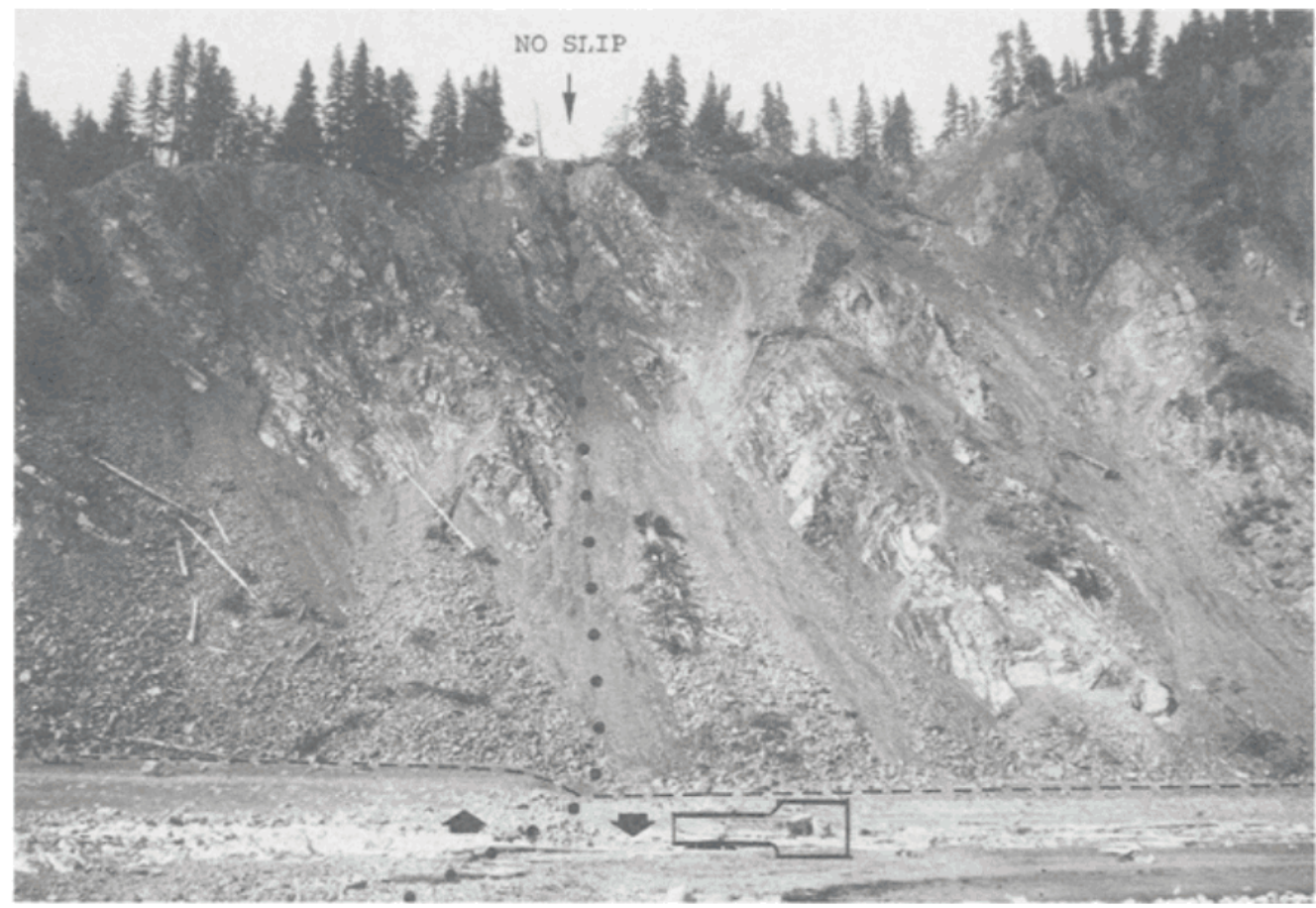

Figure 4: From Bray et al. (1994a). The Patton Bay Fault at Beach and Sea Cliff near Jeannie Point (Dotted line delineates main fault trace; Photograph by Bonilla)

Bray et al. (1994a) also sought to better understand the impact overlying material and fault dip on rupture processes. Through analysis of select case histories, Bray et al. (1994a) identified generalized rupture paths to be expected as a function of material stiffness and fault dip. Figure 5 illustrates these conclusions and affords another source with which to compare the broad results of this study. 


\section{LESS OFFSET AT GROUND SURFACE}

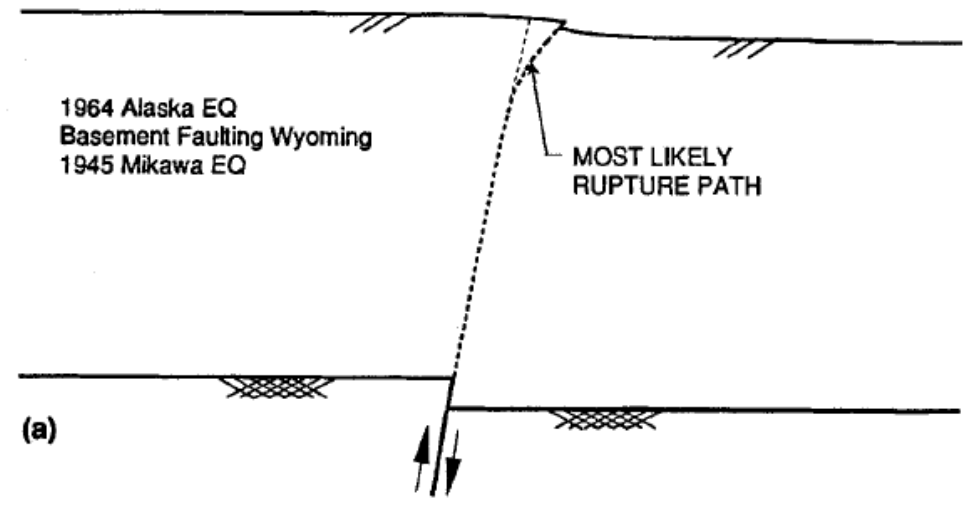

LESS OFFSET AT GROUND SURFACE
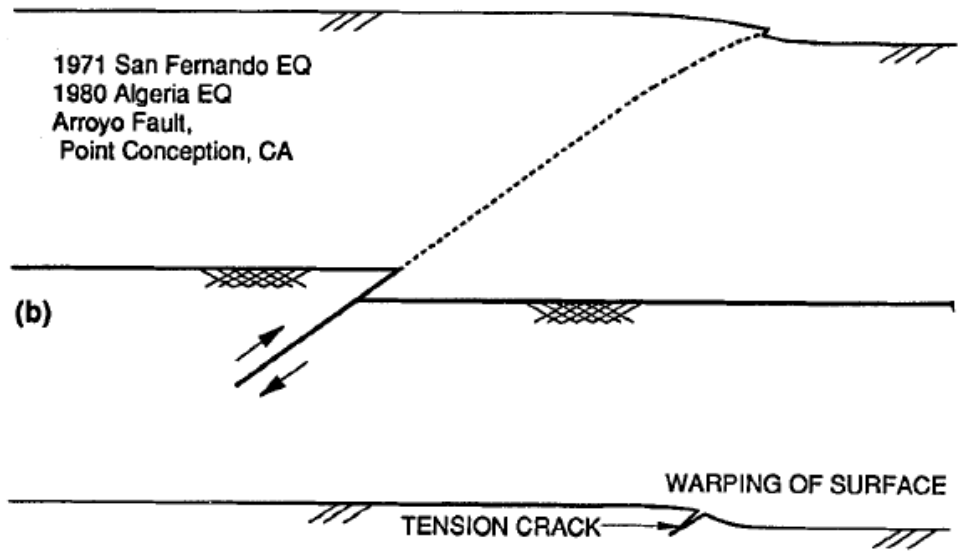

1964 Alaska EQ

1971 San Fernando EQ

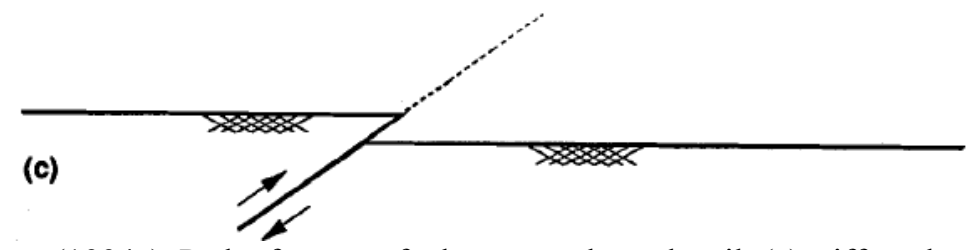

Figure 5: From Bray (1994a). Path of reverse fault rupture through soil: (a) stiff earth materials, steep dip; (b) stiff earth materials, shallow dip; (c) ductile earth materials

\subsubsection{Previous Physical and Numerical Research}

Numerical experiments performed in Bray et al. (1994b) showed that the post-failure properties of cohesive materials, specifically failure strain, largely control rupture propagation. Finite element procedures simulating reverse fault displacement at $45^{\circ}$ beneath saturated clay demonstrated that the vertical base offset was between $3.9 \%$ and $5.6 \%$ of the height of the resulting shear band. Furthermore, it was observed that with 
increased failure strain, greater basal displacement was required for surface faulting. These results are shown in Figure 6. Since increased failure strain of clay correlates to decreased stiffness and greater ductility, this suggests a similar trend as is presented in Moss et al. (2013).

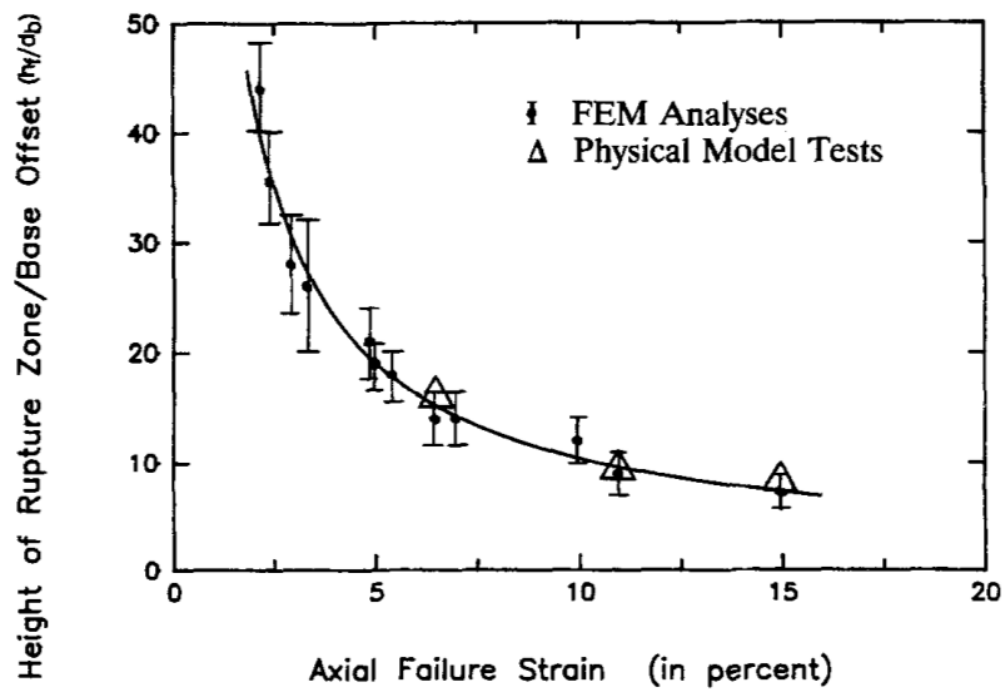

Figure 6: Finite Element prediction of normalized height of shear rupture zone as a function of clay's failure strain. From Bray et al. (1994b).

Anastasopoulos et al., (2007) developed finite element methodology to study dip-slip fault rupture through sand. The methodology was validated using centrifuge tests simulating both reverse and normal fault movement beneath dense and loose sand. In reverse faulting, the ratio of vertical basement rock displacement required for surface rupture to height of the soil profile to be $2.5 \%$ and $4.0 \%$ in dense and loose sand respectively (Figure 7). However, according to Figure 7, the difference between the normalized basement displacement needed for surface rupture in loose and dense sand above normal faults is much smaller. This indicates that the form of dip-slip movement is an important factor for predicting the probability of surface rupture. 

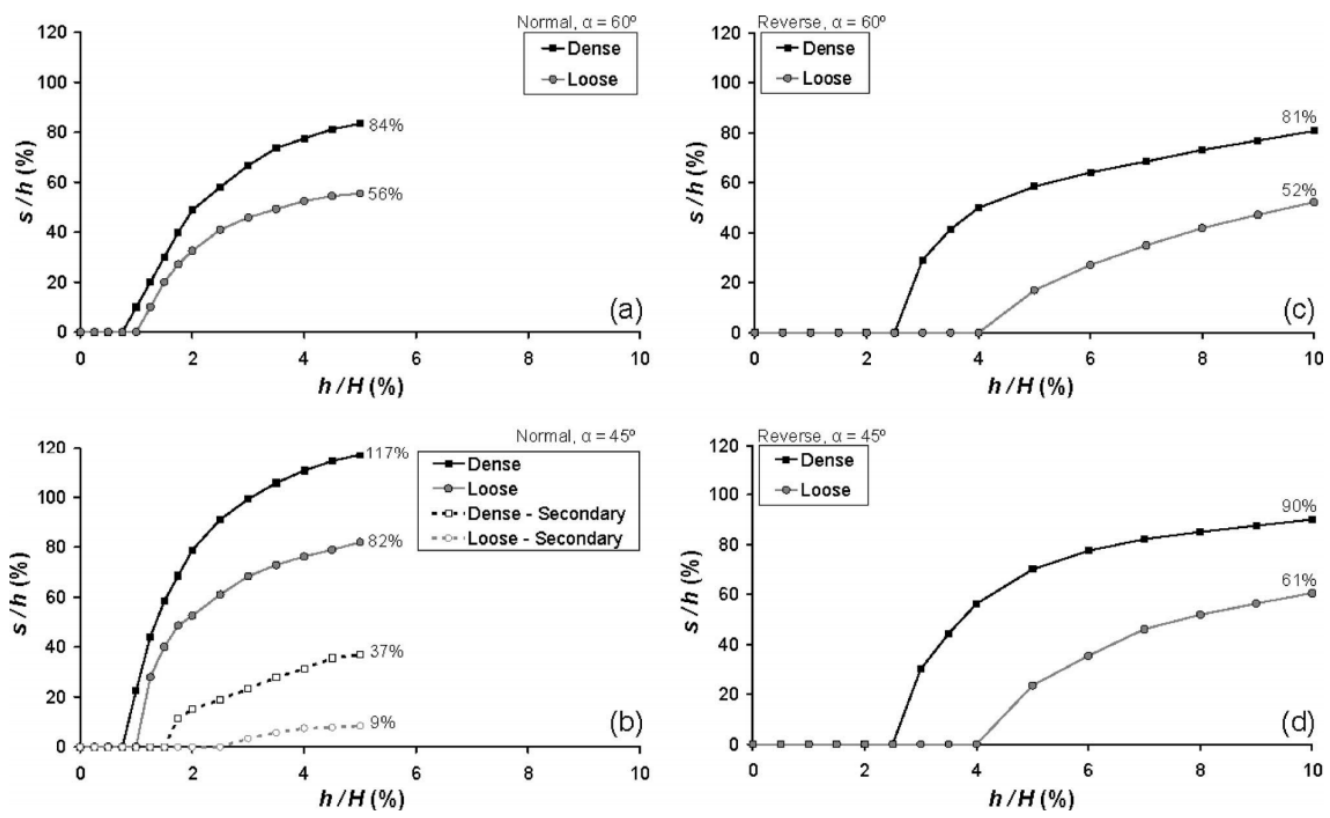

Figure 7: From Anastosopoulos et al. (2007). Normalized fault scarp height $s / h$ versus normalized bedrock displacement $h / H$ : (a) normal fault, $\alpha=60^{\circ}$; (b) normal fault, $\alpha=45^{\circ}$; (c) reverse fault, $\alpha=60^{\circ}$; and (d) reverse fault, $\alpha=45^{\circ}$ (dense sand: $\varphi=45^{\circ}, \psi=18^{\circ}$, and $\gamma_{y}=1.5 \%$; loose sand: $\varphi=32^{\circ}, \psi=3^{\circ}$, and $\gamma_{y}=3 \%$ )

Along with the previous study, the findings in Anastosopoulos et al., (2007) support the concept that rupture through both sand and clay respond similarly to an increase in material stiffness. In addition, it raises the question of exactly how material type influences the rupture process.

Recently, Oettle and Bray (2013a) used the two-dimensional, plane strain, explicit finitedifference program FLAC to conduct simulations to explore the effects of prior rupture on surface fault rupture development. They noticed that rupture tends to occur in two distinct phases. The first, broad deformation is followed by, the second, localized deformation. The instant which strain localization occurs, however, is variable. Oettle and Bray went on to show that fault rupture through previously ruptured soil results in an evolution of the stress state of the material in and around the rupture plane. Overall, they found that the presence of a pre-existing shear band promotes rupture propagation along the same 
shear band in subsequent events (Figure 8). However, distinct shear band formation occurred much more readily in stiff, dilatant materials than in loose, contractive materials. As a result, the effects of prior rupture through loose soil were minimal compared to that through stiff soil given the same initial basal displacement. With this in mind, and considering that in most natural scenarios prior failure has occurred, the experiments in this study are extended to investigate the influence of a pre-existing shear band.

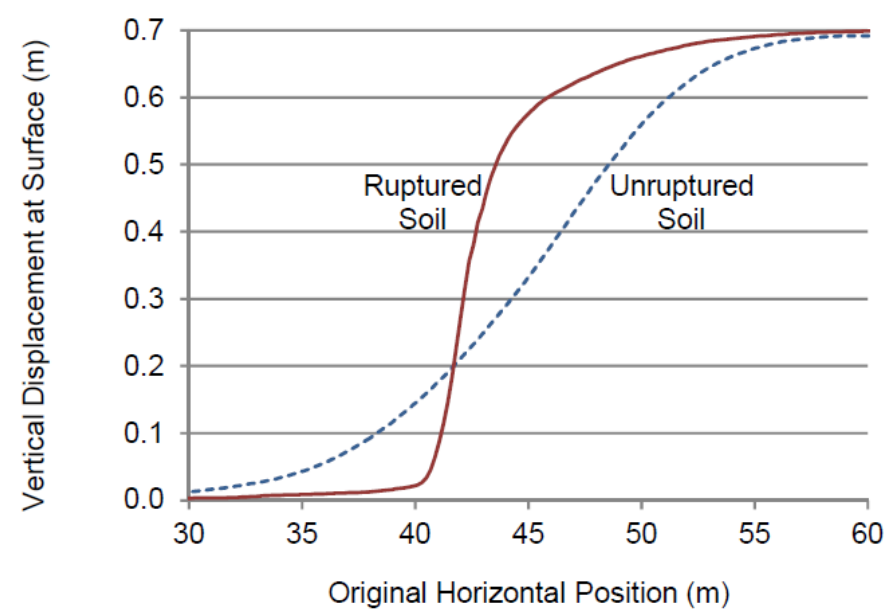

Figure 8: From Oettle and Bray (2013). Comparisons between unruptured and previously ruptured soil for the same conditions as centrifuge test 28 from Bransby et al. (2008b) (reverse fault, $60^{\circ}$ dip, 15-m soil, 2.0 $\mathrm{m}$ of previous vertical fault movement, $0.7 \mathrm{~m}$ of vertical fault movement, $\mathrm{N}_{1,60}=22, \mathrm{~K}_{0}=0.45$ ).

The models employed in Oettle and Bray (2013) were validated using physical data from Bransby et al., (2008) in which fault box experiments were performed in a centrifuge. The fault box served as a $1 / 115$ scale model of a reverse fault oriented at $60^{\circ}$ beneath 15 meters of sand at prototype scale. This translates to a soil depth of $130.4 \mathrm{~mm}$ at model scale. Although the purpose of the experiments was to understand more about fault foundation interaction, one test was performed with free-field conditions. In this test (test 28), the ratio of vertical fault displacement to the height of the soil column $(h / H)$ required 
for surface rupture was approximately $10 \%$ (Figure 9). They also observed the same general two-stage rupture process described in Oettle and Bray (2013).

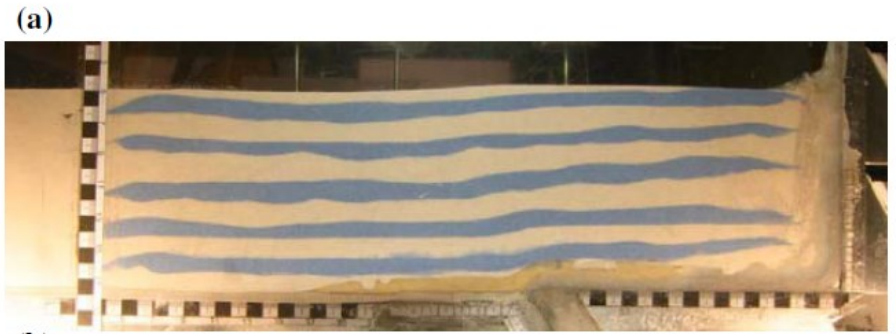

(b)

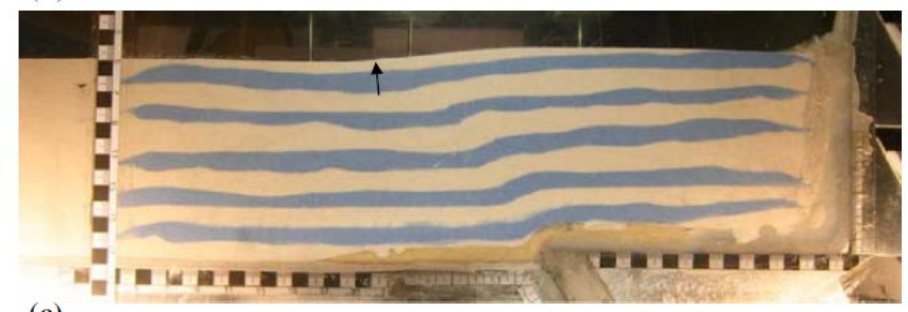

(c)

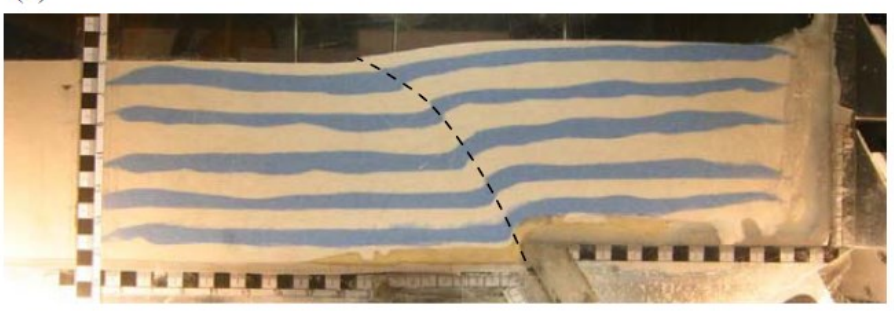

Figure 9: From Bransby et al. (2008b). Free-field reverse faulting (test 28): Images of deformed soil specimen for fault throws (a) $\mathrm{h}=0.98 \mathrm{~m} ;$ (b) $\mathrm{h}=1.52 \mathrm{~m}$ and (c) $\mathrm{h}=2.21 \mathrm{~m}$

Centrifuge tests were also conducted by Roth et al. (1981) to model the response of alluvial deposits to $45^{\circ}$ reverse and normal fault rupture. These tests are particularly pertinent because they help us gauge the influence of the rate of fault movement, a parameter which we had very little control over in the present study. In nature, dip-slip fault displacement rates difficult to predict and in theory could produce either very fast or very slow stress drops in the soil above, corresponding to dynamic or static loading, respectively. This raises the question of whether or not inertia forces significantly affect the rupture process. Surprisingly, though, the $50 \mathrm{~g}$ centrifuge tests with base displacement 
rates ranging from $1.5 \mathrm{~cm} / \mathrm{s}$ to $2.5 \mathrm{~cm} / \mathrm{s}$ from Roth et al. (1981) produced strikingly similar results as the essentially static fault box tests performed at $1 \mathrm{~g}$ by Cole and Lade (1984). Although both of these tests involved sand exclusively, clay soils tend to have their behavior dominated by inter molecular forces as opposed to gravity. Thus, it would be expected for fault movement rates to play even less of a role in rupture through clay as long as it is loaded in an undrained fashion.

The fault box procedures from Cole and Lade (1984) were primarily meant to study the shape of rupture planes as a function fault angle and material stiffness for both dense and loose sand. When modeling a $45 \mathrm{~cm}$ profile of dense sand, a complete failure surface typically developed after 1.0 to $2.0 \mathrm{~cm}$ of vertical displacement $(h / H=2.2-4.4 \%)$. Although no similar quantitative measurements were reported for loose sand, they did observe that larger vertical movements were required to develop failure surfaces in loose versus dense sand. Selected images from their tests are shown in Figure 10. 

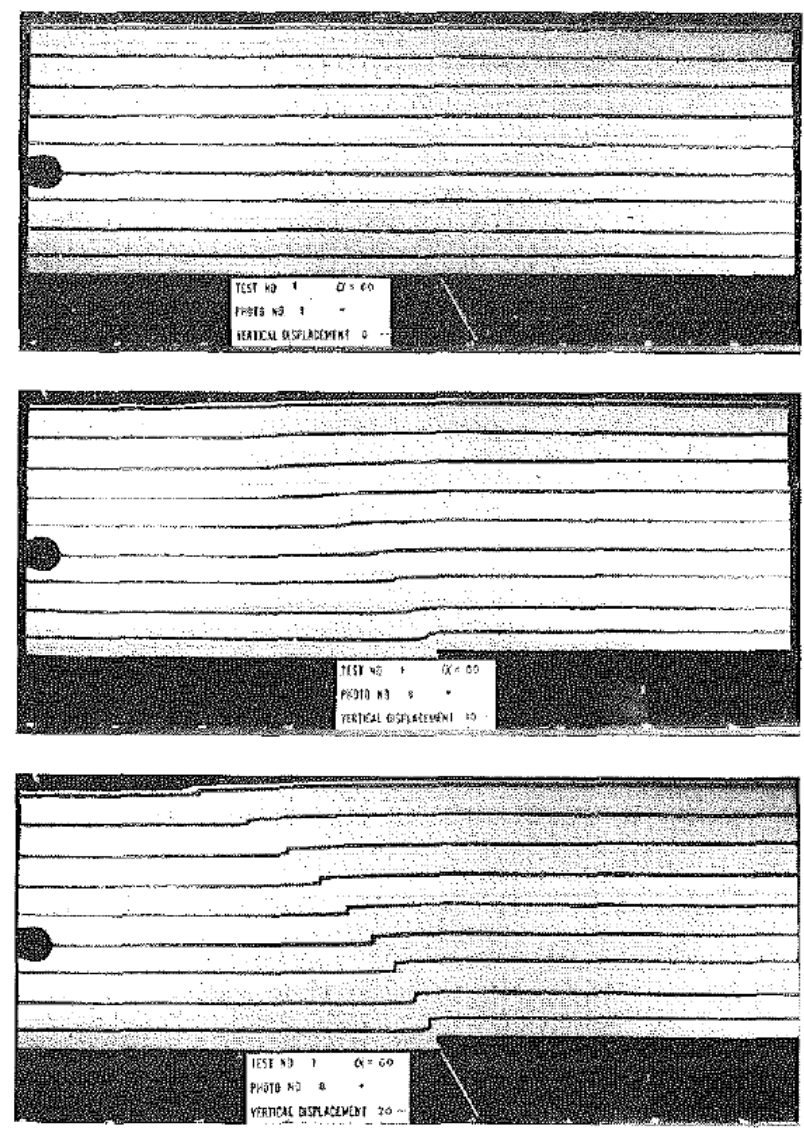

Figure 10: From Cole and Lade (1984). Photographs of three stages in a test on dense sand with dip angle $=60^{\circ}$ and reverse movement: (a) Undeformed condition; (b) Initiation of failure surface at bedrock fault:

(c) Fully developed failure surface.

Using the physical shear box tests, Cole and Lade (1984) also calibrated a numerical model which was successful in predicting the location and shape of failure planes. They note that the angle of dilation was the only soil property entering the model. This suggests that the nature of failure planes in sand are controlled by the rate of volume change, meaning the stress characteristics of a sand mass are less important. Similar results were reported by Roscoe (1970), Bransby and James (1970), James and Bransby (1971), and Wroth and Basset (1965). This is pertinent because rate of volume change is described by the angle of dilation which is related to the velocity characteristics of a material. Therefore, considering that shear wave velocity can be used as a proxy for 
material stiffness through the small strain shear modulus, it follows that stiffness should indicate the potential for surface rupture in cohesionless soil in the same manner described by Moss et al. (2013).

Although it is not covered herein, the water content is another potentially impactful near surface soil property (Johansson and Konagai, 2006). In a deviance from the traditional 2-D plane strain approach, like in this study, physical and numerical axi-symmetric fault experiments revealed that deformations localized at a later stage in wet sand as opposed to dry. Given that the extent to which water content affects rupture propagation is not yet quantified, it is still could serve as a possible explanation for any discrepancies observed between the comparatively wet clay and dry sand in this paper.

\subsection{Project Scope}

The motivation of this paper was to provide insight into what near surface geologic parameters are most critical for assessing hazard due to surface rupture. Specifically, the study aimed to investigate the influence of material stiffness on rupture propagation through materials with differing rupture histories and cohesive properties. With respect to previous work, the objective was to present physical evidence that either qualitatively supports or refutes the case-study statistics presented in Moss et al. (2013) concerning reverse faults as well as the numerical modeling results from Oettle and Bray (2013).

A shear box apparatus was constructed to simulate reverse fault rupture through loose and dense sand as well as soft and stiff clay. The apparatus is unique because it allows for the characteristic shear wave velocity to be measured directly before the soil is disturbed. Similar experiments have been performed in the past but never with the primary goal of 
investigating the relative effects of material stiffness, cohesion, and rupture history on fault propagation.

The following is a list of project goals:

- Construct a testing apparatus capable of mimicking idealized reverse fault rupture beneath a native soil deposit

- Conduct experiments on loose sand, dense sand, soft clay and stiff clay

- Obtain reliable shear wave velocity data for each material

- Correlate the amount of basal displacement required to initiate surface rupture with shear wave velocity, cohesion and rupture history

- Determine which parameters play the greatest role in probability of surface rupture 


\subsection{Organization of Thesis}

The subsequent chapters are organized as follows:

Chapter 2 - Provides details about the fault shear box design objectives, alternatives and methods which led to the final approach. The construction of the box is also described in a manner that accentuates the trial and error nature of the process.

Chapter 3 - Covers what data was taken and how. Includes an in depth itemization of the instrumentation and software used as well as how it was implemented. This is where the shear wave velocity determination procedure is presented.

Chapter 4 - Documents the steps taken to prepare and run each experiment. The chapter focuses on the aspects of testing that differed experiment to experiment due to the variance in materials.

Chapter 5 - A broad presentation of the results of the study. This includes select images captured from video records as well as tables and charts which help to illustrate trends.

Chapter 6 - Provides and interpretation of the results and discusses the sources of error encountered during testing. The logic used to draw conclusions from the data is also covered here. 


\section{Fault Box Design, Construction, and Instrumentation}

\subsection{Apparatus Design}

\subsubsection{Reference Description}

The shear box is an assembly of two main sections: one static and one moving. The moving section resides within the static section and includes the slip plane of the hanging wall and the rear wall of the apparatus. The static section consists of the footwall slip plane, both side walls and the front wall. Henceforth, the prospective from which descriptions are made will be from the moving end (rear), facing the static end (front). An image of the shear box is shown below in Figure 11 along with a simplified reference image in Figure 12. 


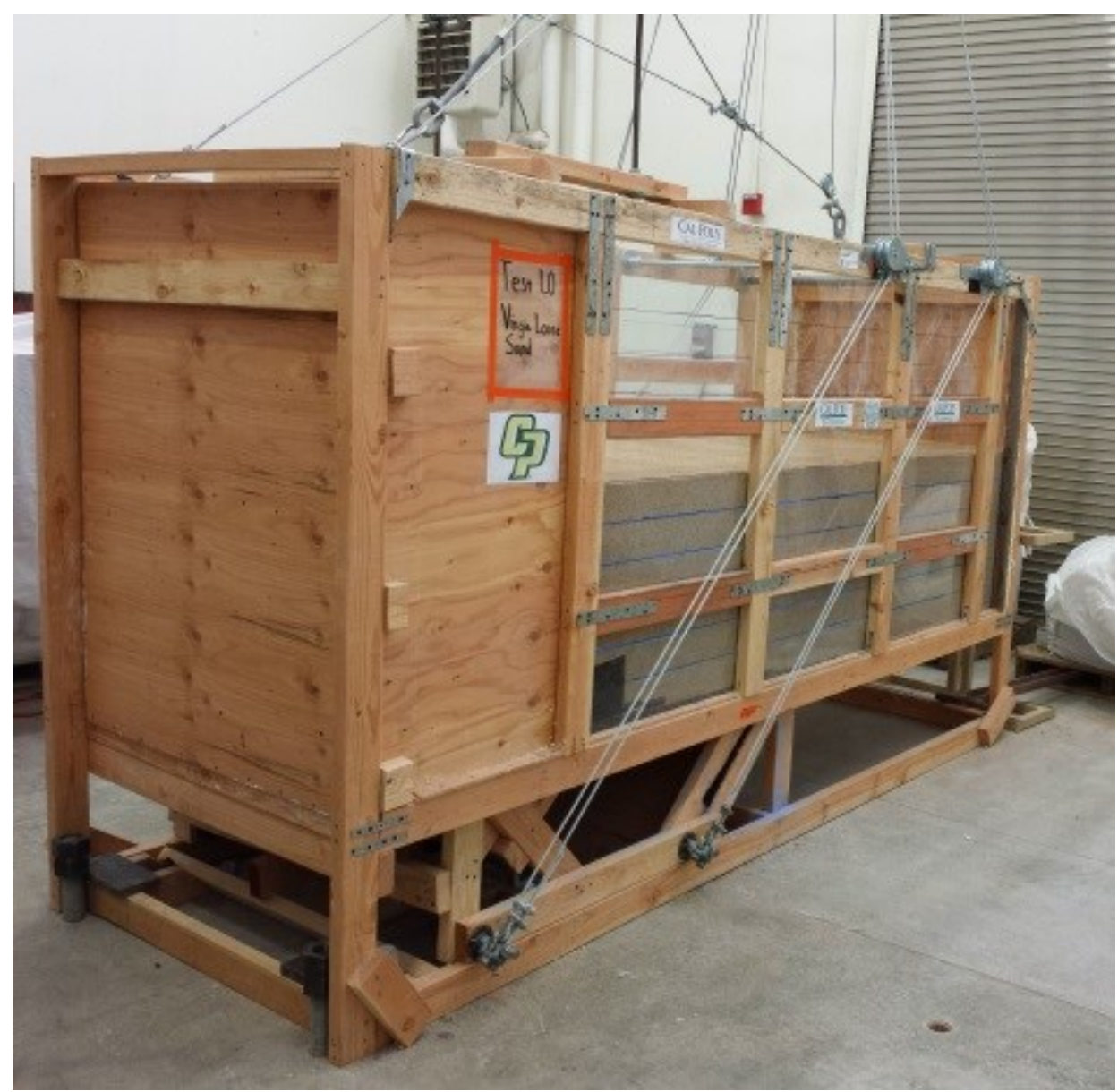

Figure 11: Fault box test apparatus located in the Parson's Earthquake Lab at Cal Poly, San Luis Obispo.

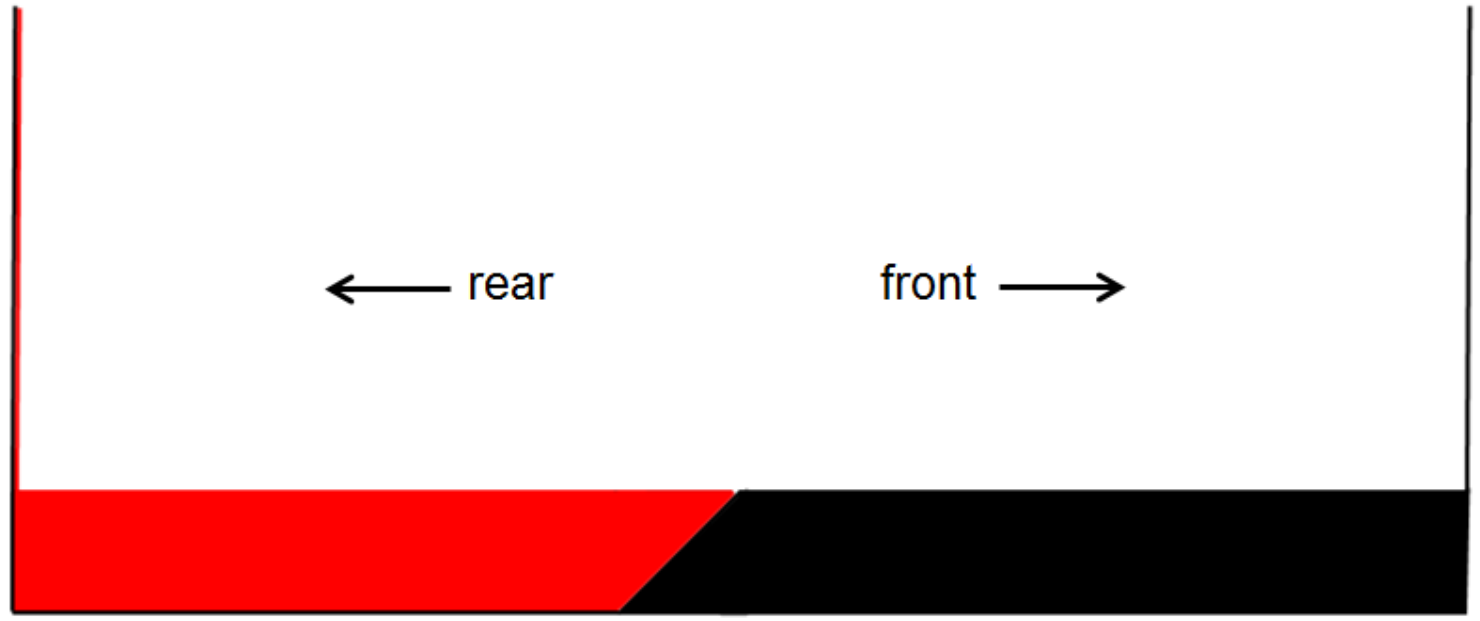

Figure 12: Simplified representation of the fault box showing only the length (horizontal) and height (vertical) dimensions. The red area is the moving section and the black area is the static section. 


\subsubsection{Dimensions of the Soil Profile}

The shear-box apparatus used for testing was designed to replicate reverse fault rupture beneath a soil profile of $60 \mathrm{~cm}$. The plan view dimensions of the profile are 1 meter wide by 3 meters long and were selected based on two principles. The first was to ensure boundary effects at the ends of the box would remain negligible. A Boussinesq approximation was adapted to calibrate the design accordingly as shown in Figure 13 and Table 1. The second principle concerned the potential to record accurate shear wave velocity data. Although a more narrow apparatus would have been more practical in terms of construction and operation due to the smaller associated loads, it may have increased the tendency of shear waves to travel through the walls of the apparatus rather than the soil profile itself. Thus, a full meter width was initially selected to improve the chances that acceptable quality shear wave velocity data could be acquired. 

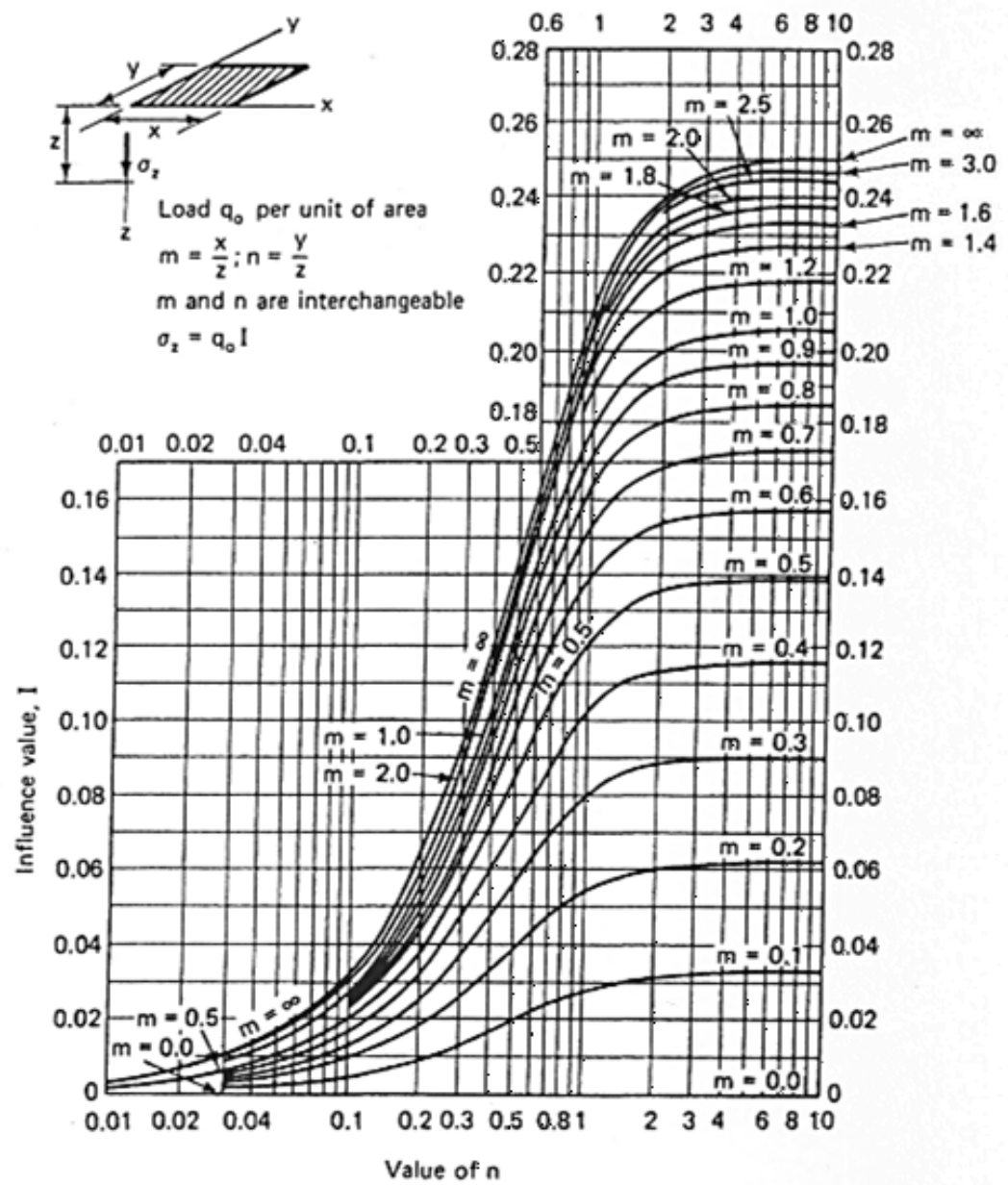

Figure 13: Influence value or stress under corner under uniformly loaded rectangular area. (From Holtz and Kovacs, 1981)

For the stress distribution analysis, a target influence value of $\mathrm{I}_{\text {total }} \leq 15 \%$ was established based on the assumption that a $15 \%$ increase in horizontal stress would not influence soil behavior enough to significantly affect the development of the rupture plane. Thus, with a known target influence value as well as the predetermined soil depth and width, the appropriate length of the fault box was estimated through an iterative technique.

Figure 13 gives the influence value beneath the corner of a rectangular loaded area. To find the influence value beneath the center, the area must first be broken into smaller parts. Those parts must consist of rectangular areas which sum to the total area over 
which load is applied and also share a corner at the geometric center of the total area. Following, the individual influence values of each area $\left(\mathrm{I}_{\mathrm{i}}\right)$ are summed to give the total influence beneath the center. For this case, the total area was the portion of the rear wall which was in contact with soil. Hence, the procedure was carried out as follows:

\section{Given:}

- $1 / 2$ width of box, $x=0.50 \mathrm{~m}$

- $1 / 2$ depth of soil in box, $y=0.30 \mathrm{~m}$

- Target total influence, $\mathrm{I}_{\text {total }} \leq 0.15$

Find:

- Desired length of box, $\mathrm{z}$

Table 1: Sample iterations performed to estimate the stress transferred across the fault box.

\begin{tabular}{|c|c|c|c|c|}
\hline Length $(\mathrm{m})$ & $\mathrm{m}=\mathrm{x} / \mathrm{z}$ & $\mathrm{n}=\mathrm{y} / \mathrm{z}$ & $\mathrm{I}_{\mathrm{i}}$ & $\mathrm{I}_{\text {total }}=\sum \mathrm{I}_{\mathrm{i}}$ \\
\hline 2.0 & 0.5 & 0.3 & $5.6 \%$ & $22.4 \%$ \\
\hline 3.0 & 0.33 & 0.20 & $2.75 \%$ & $11.0 \%$ \\
\hline
\end{tabular}

According to Table 1, a fault box measuring 1 meter wide by 3 meters long with 0.6 meters of soil would transfer approximately $11 \%$ of the horizontal stress imposed on the soil to the opposite wall before failure occurred. This is a particularly rough estimate because it assumes an infinite half-space with the free-space boundary at the rear wall. In reality, there was no true infinite half-space and the free-space boundary was at the soil surface. That being said, the goal of this analysis was only to provide a meaningful indication that the additional horizontal stress developed during operation did not significantly alter the properties of the soil in any of the test cases. To provide additional evidence as to whether or not this method is valid, we can also compare the dimensions of this fault box to that of similar devices employed in past studies. Using the same method as outlined above, the influence values corresponding to the experimental setups from Bransby et al., (2008) and Cole and Lade (1984) are 20\% and 3.2\%, respectively. 
This shows that the scaled fault box dimensions herein are within reasonable range. Considering this, we assume that the rupture path was not inadvertently influenced.

\subsubsection{Side Wall Friction}

Upon the establishment of preliminary dimensions for the soil profile, the potential effects of wall friction were evaluated. This is discussed in detail by Bransby and Smith (1975) through a physical assessment and Jayasree et al. (2012) through a numerical assessment. Both studies provide an overview of tank wall friction implications for tests concerning the bearing capacity of footings and lateral earth pressures on retaining walls. Their general conclusions, which are pertinent to this study only by analogy, are that wall friction does affect lateral and bearing forces in plane-strain models. This is especially true for high soil friction angles and high interface friction angles. Thus, to minimize epistemic error, they both make recommendations to increase tank width and decrease wall roughness.

The Plexiglass from the side walls of the fault box is much smoother than the concrete tank walls analyzed by Jayasree et al. (2012). Hence, tests on sand did not seem likely to be noticeably altered by wall friction as long as the width of the profile was large enough. This was assumed to be the case based on comparisons with Cole and Lade (1984). Despite only $15 \mathrm{~cm}$ wide fault box, they recorded only minor deviations in deformation patterns during tests on dense sand with an interface friction angle of approximately $25^{\circ}$. This suggested that the aforementioned soil profile dimensions were at least adequate for sand. 
For clay, the main concern was that adhesion might overcome cohesion. This would not likely have affected the overall shape of the rupture plane as much as our ability to view it through the Plexiglass. Thus, as a precaution, the Plexiglass walls were lubricated (with PAM cooking spray) before each flight of clay was placed to reduce adhesion.

\subsection{Construction Materials}

The fault box apparatus was constructed primarily out of timber with steel reinforcing in select locations. The placement of steel reinforcing was determined by identifying problem areas during preliminary weighted test runs and addressing them accordingly. As a result, the final design included steel cross-braces (Figure 14) spanning the top sections between the pulleys shown in Figure 15 to compensate for the excessive forces and moments that developed in those regions. In addition, steel strap ties cover most connections in the side wall frames to limit out of plane deformation. 


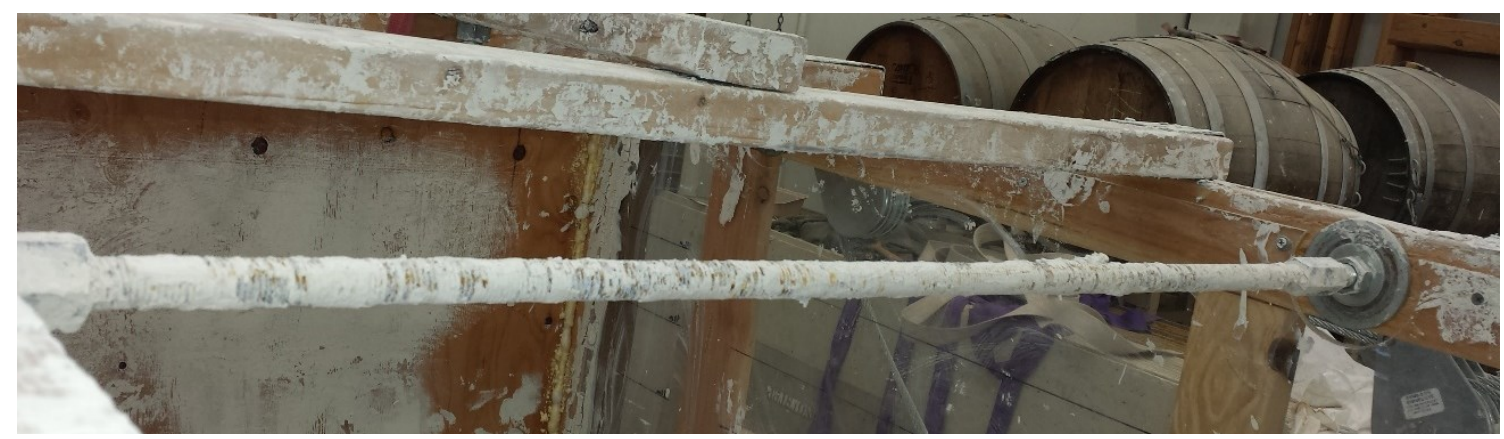

(a)

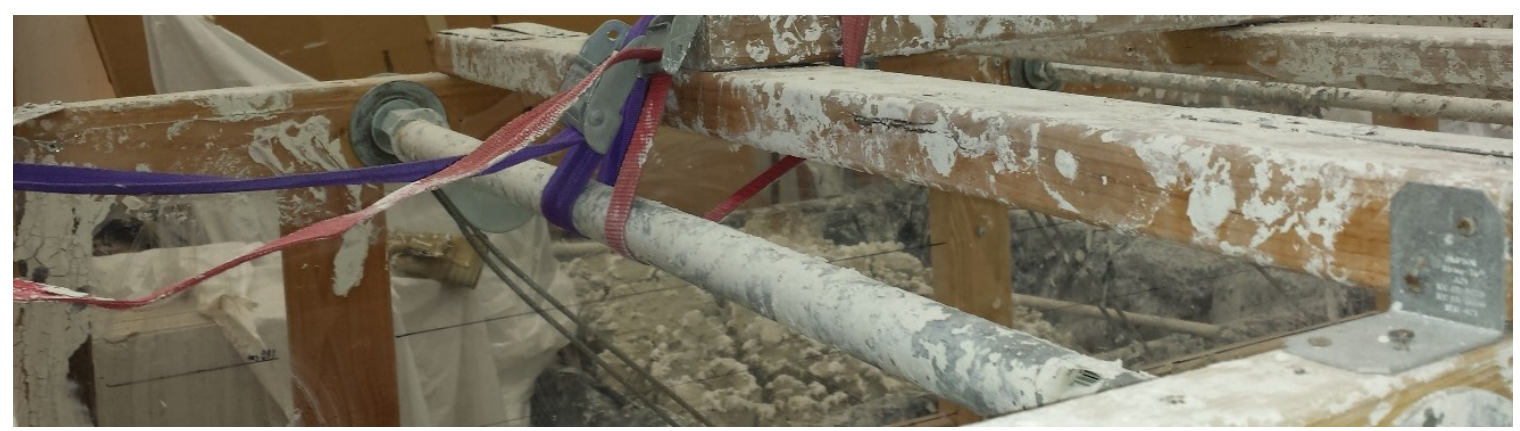

(b)

Figure 14: Steel cross-braces installed for bending moment resistance at hanging swivel eye double pulley connection locations. (a) Rear; (b) Front 


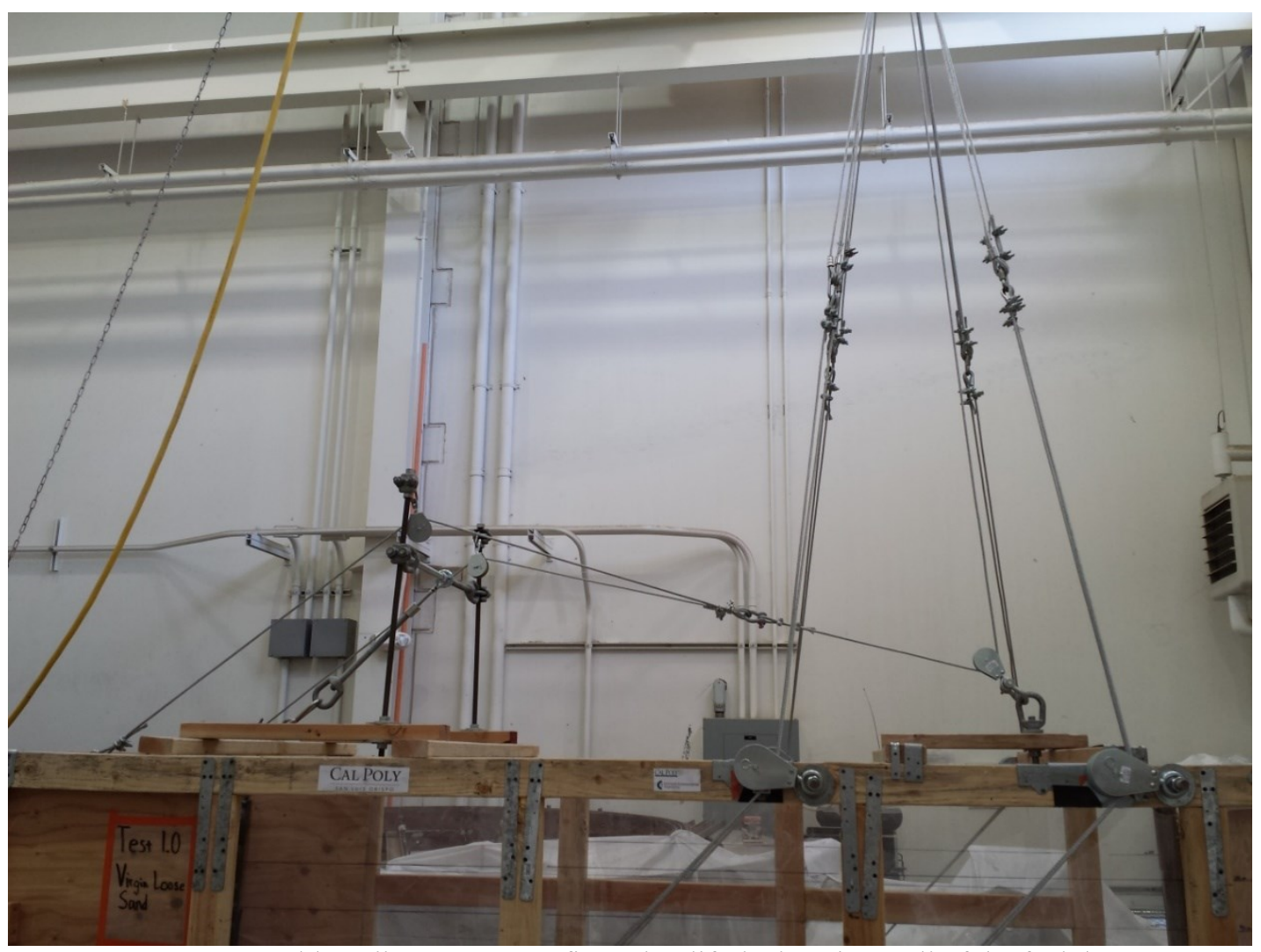

Figure 15: Cable pulley system configured to lift the hanging wall of the fault box.

Floor bolts were installed on the rear end of the fault box (Figure 16) to help prevent the static portion from lifting off the ground during operation. Unfortunately, there were no female sockets available in the floor of the lab near the front end of the box.

Consequently, scrap metal was used to supply the additional weight needed to keep that end on the floor (Figure 17). 


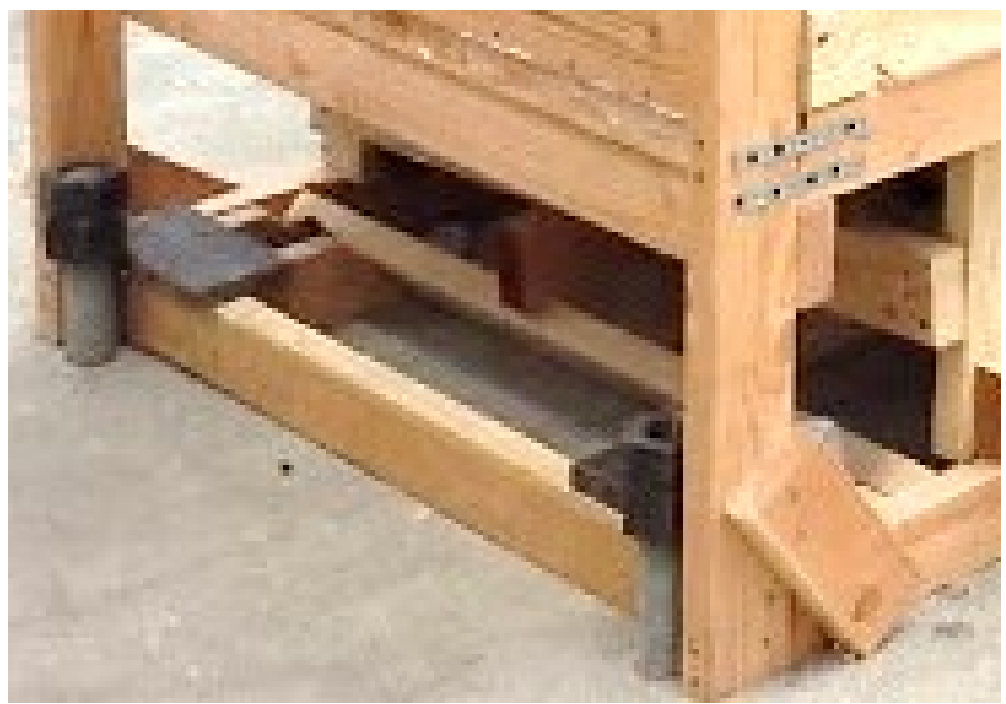

Figure 16: Floor bolts anchoring the rear end of the fault box to the floor of the Parson's Earthquake Lab.

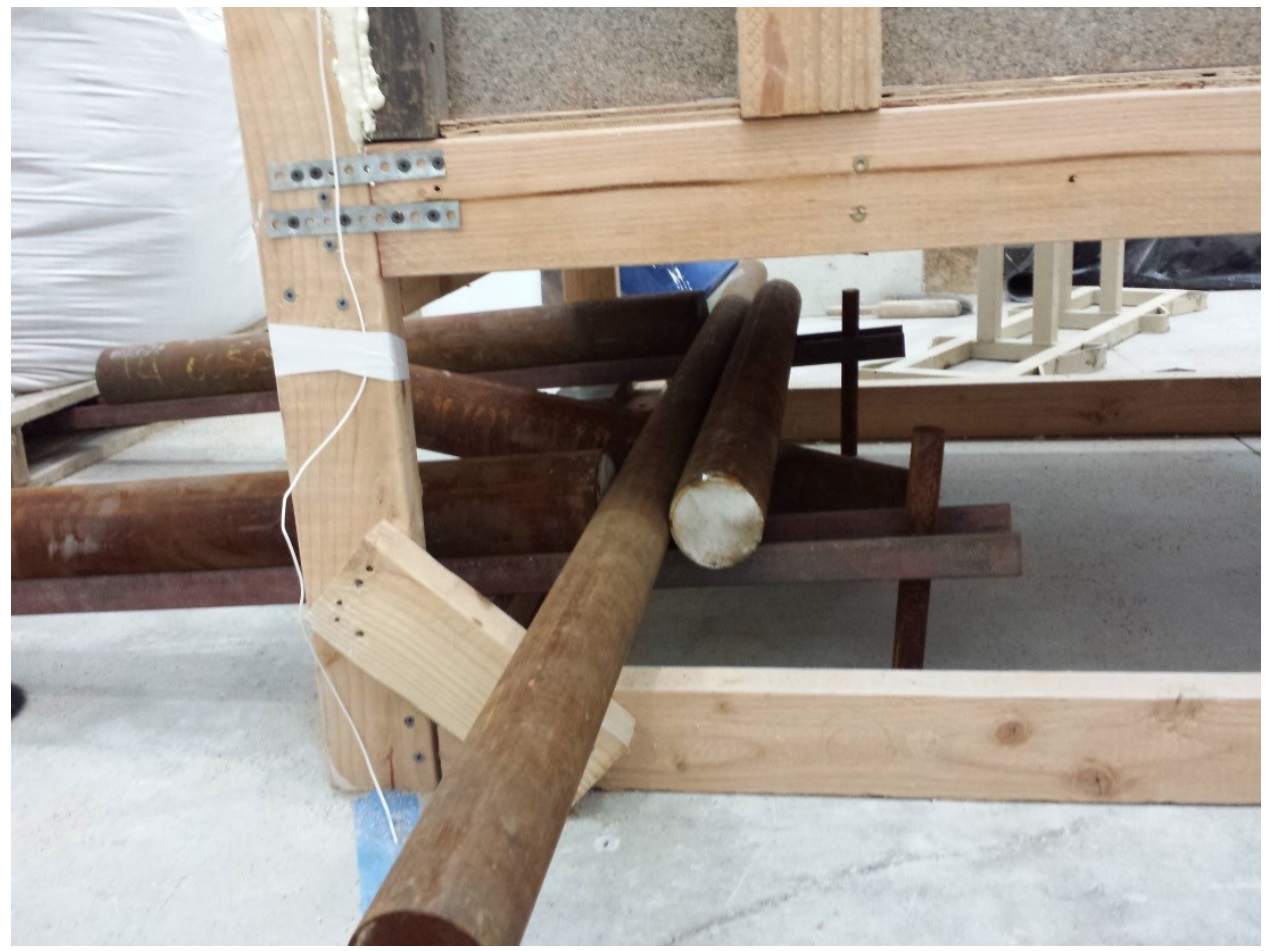

Figure 17: Weighting system used to anchor the front end of the fault box to the floor of the Parson's Earthquake Lab.

The majority of the side walls were made of Plexiglass to afford a view of the rupture plane. Sanded plywood was installed for the remaining half-meter portion of the side walls nearest the rear end of the box to ensure a tight seal could be maintained along the 
boundaries between the side and the moving rear wall. This would have been problematic using Plexiglass because it is more susceptible to out of plane deformation than plywood.

Both surfaces of the slip plane consisted of sanded plywood covered in UMHW Slick Tape to reduce friction. For additional guidance, a set of $2 \times 4$ 's were in the same orientation as the slip plane but offset one meter to the rear, with one fixed to the static frame and one to the moving frame. They were also sanded and covered in UMHW Slick Tape.

Two layers of 1/4" soft adhesive backed F13 felt sheeting (Figure 18) were used to prevent sand from escaping through the advancing boundaries. The first was stapled to the base and rear wall edges of the moving section and was forced into the gap between the two surfaces. The second layer of felt was wrapped around 1" thick adhesive Neoprene foam stripping (Figure 19) and pressed against the walls of the box before being secured directly on top of the first layer. Felt spanning the embedded trace of the slip plane was also fastened to the fixed end of the box to prevent material entering the area of the slip plane. Finally, the transition from plywood to Plexiglass along the rear and of the side walls was bridged with a sand tight seal using adhesive backed felt covered in slick tape. 


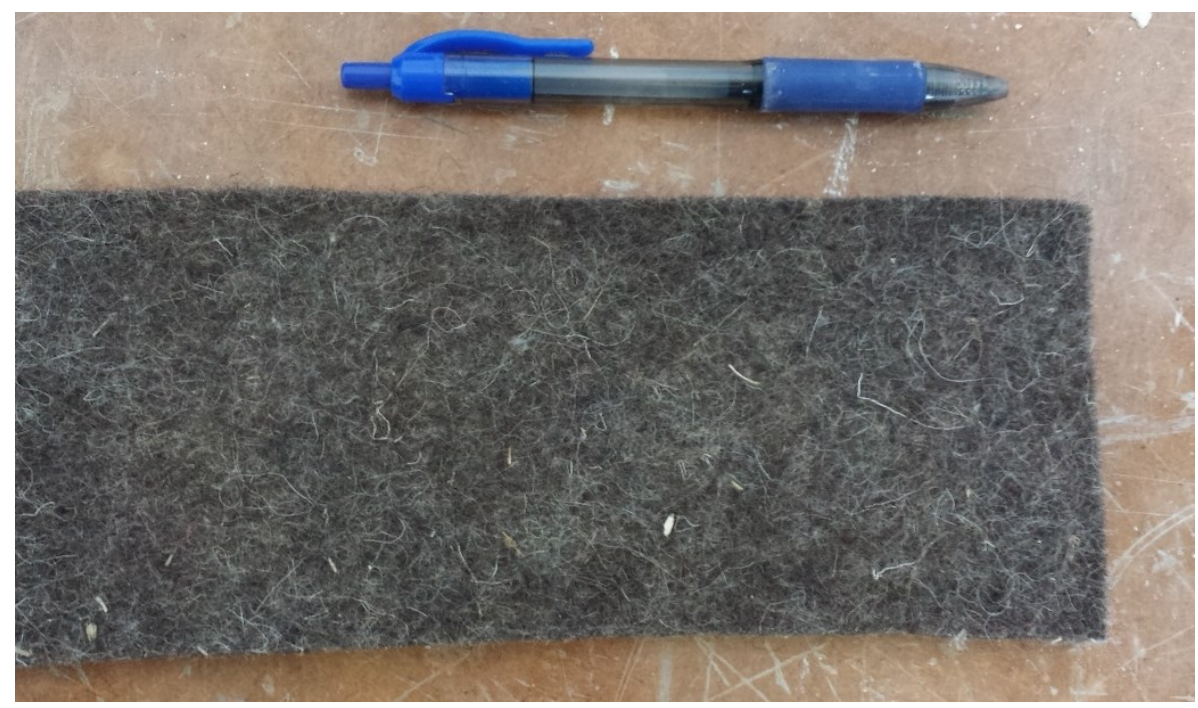

(a)

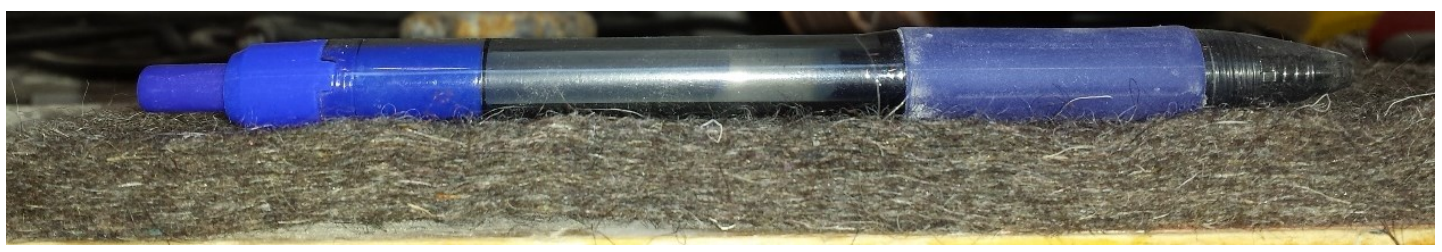

(b)

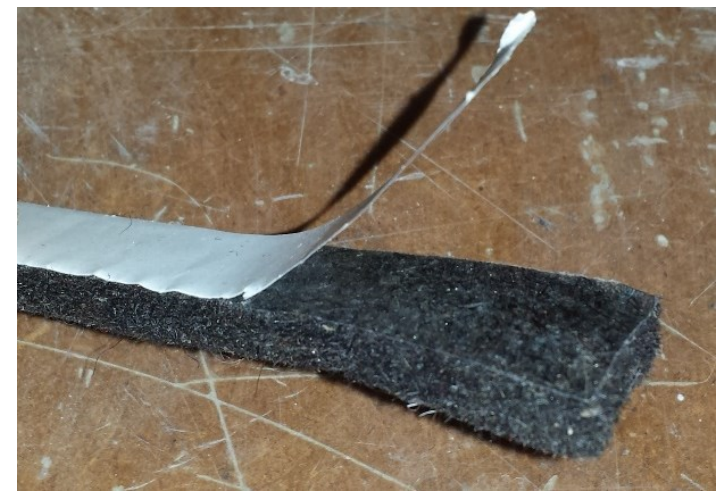

(c)

Figure 18: 1/4" soft F13 felt sheeting.. (a) Top view; (b) Side view; (c) Adhesive backing. 


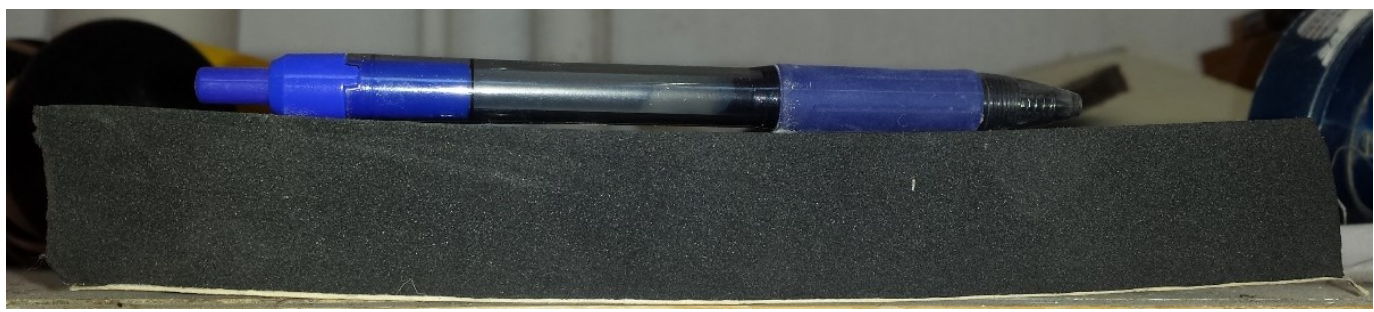

(a)

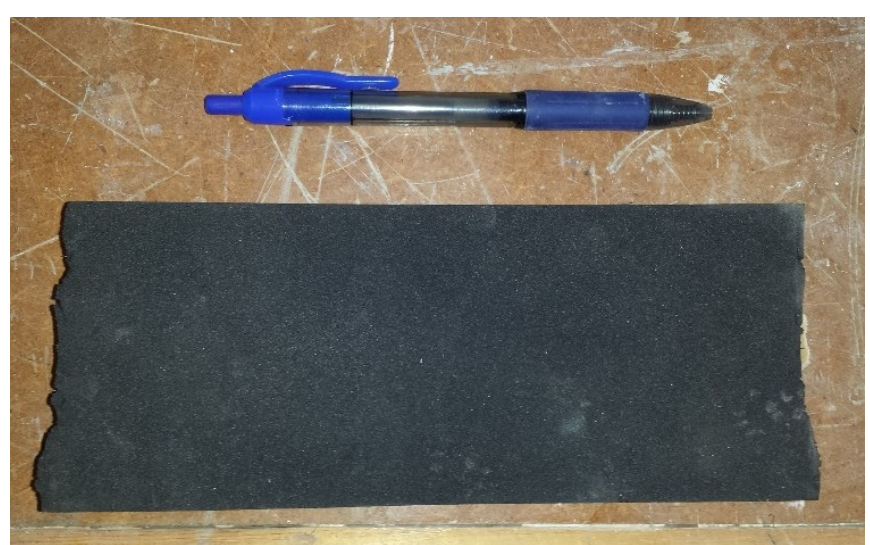

(b)

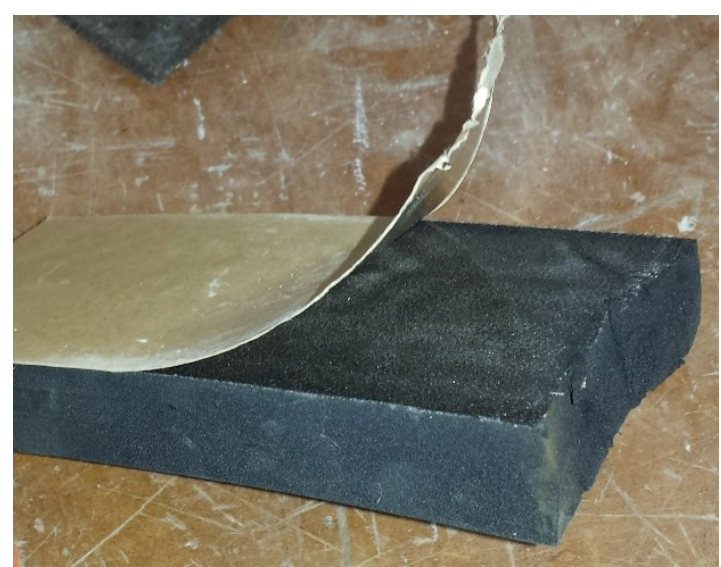

(c)

Figure 19: 1" thick adhesive Neoprene foam stripping. (a) Side view; (b) Top view; (c) Adhesive backing.

When testing the clay, Visqueen was wrapped over the felt liners and wooden surfaces.

The felt along the slip plane, however, was removed and bridged by Visqeen which was folded like baffles and in such a manner that it did not impede basal displacement yet still prevented material and moisture from escaping. 


\subsection{Loading Mechanism}

A 3 ton ceiling crane, shown in Figure 20, functioned as the loading device for the system. The main objectives of the lifting design were to direct forces such that the hanging wall was pulled at a $45^{\circ}$ angle while the crane applied a purely vertical load.

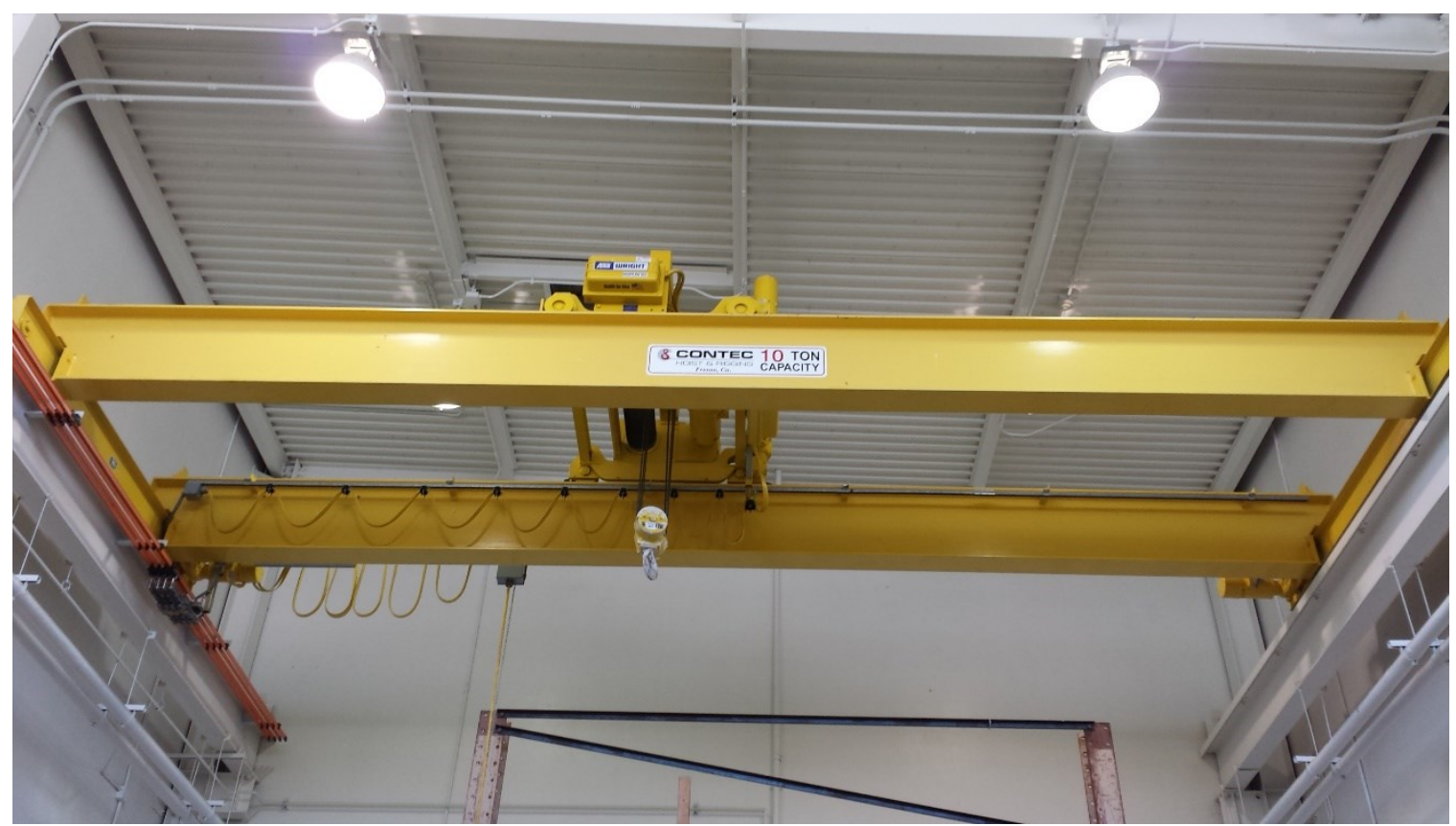

Figure 20: 3 ton ceiling crane located in the Parson's Earthquake Engineering Laboratory at Cal Poly, San Luis Obispo.

1/4" zinc coated steel rope cables were connected at six locations and extended at least 75 $\mathrm{cm}$ at a $45^{\circ}$ angle before being redirected to the crane through the arrangement of pulleys shown in Figure 15. This allowed for a maximum basal displacement of $75 \mathrm{~cm}$.

As shown in Figure 15, the cables fixed to the top of the rear (moving) wall were drawn through two hanging swivel-eye pulleys before joining to form a single cable. The single cable then ran through yet another hanging swivel-eye pulley located directly below the crane. The cables fixed to the base of the moving end were double strands to meet the 
necessary tensile load capacity and were fed through hanging swivel-eye double pulleys to the crane.

The load from the cables is transferred to the hanging wall using two connection styles. At the base, 3/4" steel threaded rods are bolted through the moving frame and fastened to the cables with clamping U-bolts. At the top, self-tapping steel eyebolts serve as anchors for the cables.

\subsection{Similitude}

The similitude relationships presented below are useful for understanding prototype, or full scale, behavior based on the results of a scaled model experiment. Unfortunately, they severely limit material selection and render the experiments conducted on the $2 / 16$ Monterey Sand purely qualitative in nature. The clay, on the other hand, was mixed to produce properly scaled soil parameters. Thus, if the effects due to factors not quantified herein were accounted for, such as adhesion between the soil and side walls, prototype scale interpretations regarding the clay results could be made. That being said, no conclusions concerning behavior at the prototype scale are drawn in this study.

A number of approaches for deriving scaling relationships have been developed for scale modeling applications. Meymand (1998) highlights three approaches in a comprehensive review of similitude in geotechnical applications: dimensional analysis, similitude theory and the method of governing equations. The most simplistic, dimensional analysis, denotes dimensionally homogeneous equations in terms of dimensionless factors based on mass, length and time. Similitude theory exploits the Buckingham Pi Theorem and expands on dimensional analysis by accounting for the unique forces acting in a system 
for the formation of dimensionless terms. The most complex, the method of governing equations, requires the theoretical transformation of a system to be described by a complex differential equation which is converted to a non-dimensional form. For this study, dimensional analysis is used to develop a relationship between the prototype and model scales.

The prototype scale for the apparatus is related to the actual dimensions through the geometric scaling factor $\lambda$. Table 2 below shows engineering parameters of interest in terms of $\lambda$ (adapted from Iai. 1989; Meymand, 1998).

Table 2: Scale factors of engineering variables in terms of geometric scaling factor $(\lambda)$ (adapted from Iai. 1989; Meymand, 1998)

\begin{tabular}{|c|c|c|}
\hline Variable & Scale Factor & For $\lambda=10$ \\
\hline Soil Density & 1 & 1 \\
\hline Stiffness & $\lambda^{2}$ & 100 \\
\hline Shear Wave Velocity & $\lambda^{1 / 2}$ & 3.162 \\
\hline Length & $\lambda$ & 10 \\
\hline Strain & 1 & 1 \\
\hline Time & $\lambda^{1 / 2}$ & 3.162 \\
\hline
\end{tabular}

\subsection{Noteworthy Complications}

The availability of the 3 ton ceiling crane in the Cal Poly Parson's Earthquake Laboratory presented a convenient opportunity to learn how best to power a fault box experiment with an overhead crane. Unfortunately, using the ceiling crane to move the hanging wall without causing detrimental deformations or vibrations in the soil proved to be particularly challenging. Although a properly functioning apparatus was eventually developed it should be noted that hydraulic actuators, which have been used prior research (e.g. Cole and Lade, 1984; Bransby et al., 2008), may have made for a more simplistic design. 
Initially, four 5/16" steel cables were redirected with a series of cable guides beginning at the base of the soil profile instead of at the top of the apparatus with pulleys as shown in Figure 11, Figure 15 and described earlier. Unfortunately, using a single line of cable through cable guides caused vibrations during preliminary trails which would have densified the loose sand. In addition, the 5/16" cables sustained damage during each weighted test run because they were traversing smaller angles than their rated bend radius. To overcome this, the $5 / 16$ " cables were replaced with doubled $1 / 4$ " cables which had a smaller bend radius and were also zinc coated to avoid corrosion. Doubling the cables not only avoided damage but drastically reduced vibrations in the cable guides.

Although doubled cables reduced vibrations, redirecting them at the base caused too much temporary out of plane deformation for a sand tight seal to be maintained and ultimately led to pervasive leaks at the edges of the moving end. Thus, after experimenting with a number of configurations, it became clear that cable guides simply were not suitable for this application and were abandoned. Instead, four steel hanging swivel-eye double pulleys were positioned in the appropriate locations on the top of the apparatus.

Hanging swivel-eye pulleys still allowed for minimal vibrations and were also advantageous because the orientation of each pulley adapted as the angle between it and the connection to the crane decreased during operation. This eliminated rubbing between the cables and fixed surfaces which was difficult to avoid with cable guides and mounted pulleys. The true advantage of the pulleys, however, was that they allowed for the complete redirection of the cables at just one location due to their higher load capacity. Thus, there were no longer loads causing deformation at the base of the profile and the 
system could function without losing or inadvertently disturbing any material being tested. 


\section{Data Acquisition}

\subsection{Instrumentation}

Data acquisition was carried out using a PC with National Instruments NI-DAQ data acquisition software and LabView installed. The PC was also equipped with a National Instruments SCXI 1001 chassis which accommodates up to twelve SCXI signal conditioning units. Images of the data acquisition system are shown in Figure 21.

(a)

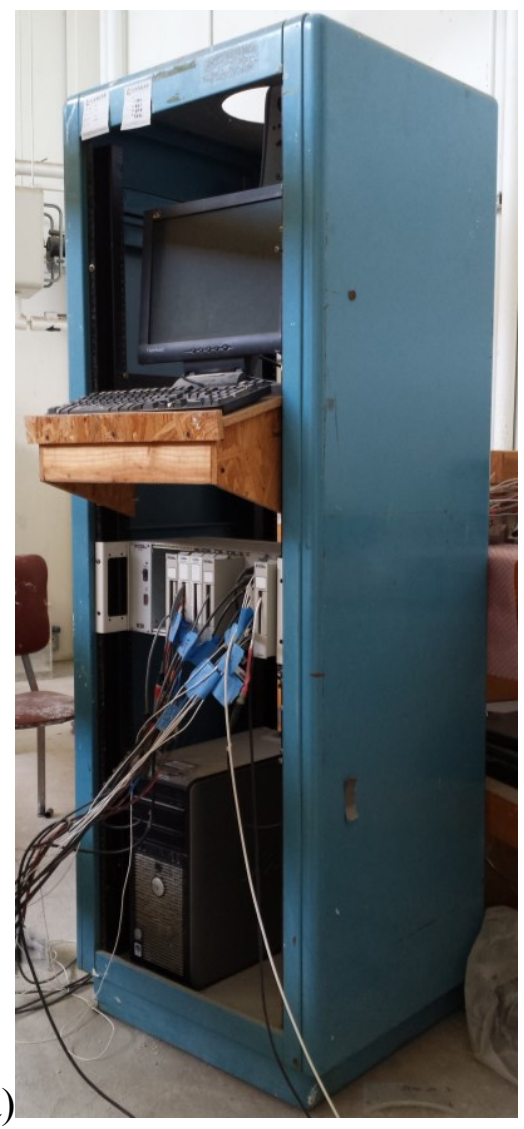

(b)

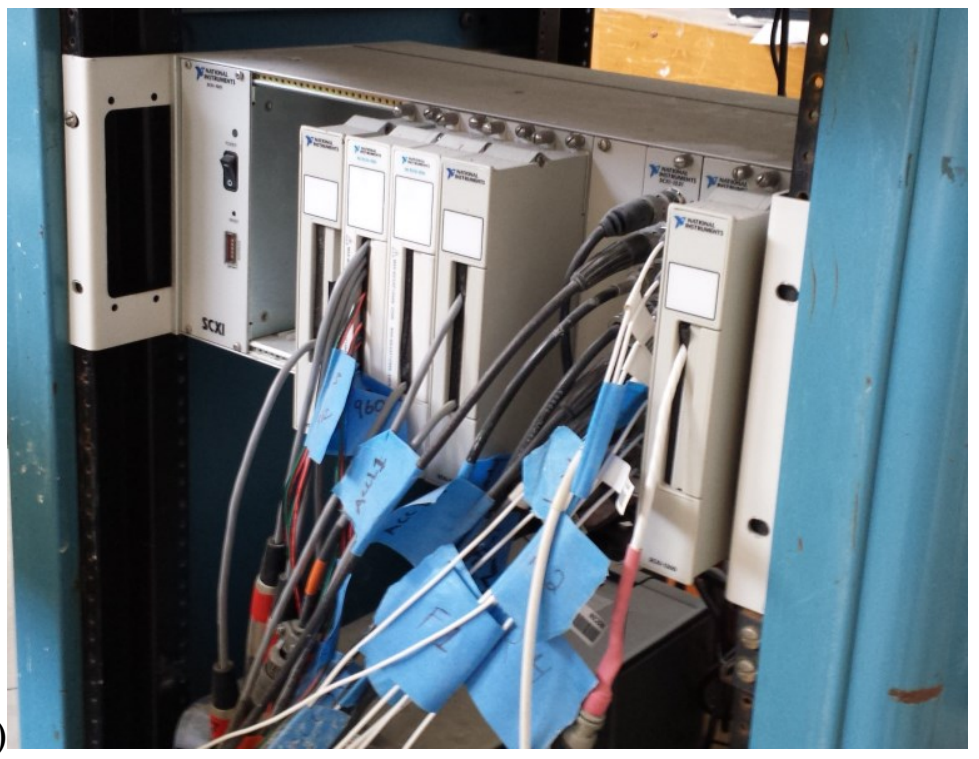

Figure 21: (a) Complete data acquisition setup; (b) Close up of National Instruments SCXI 1001 chassis. 


\subsubsection{Acceleration Data}

Before each run the average shear wave velocity of the soil profile was determined using a vertical array of two 393B04 Integrated Circuit Piezoelectric accelerometers. One was mounted at the base of the soil on a $2 \times 4$ wrapped in insulating tape to protect against vibrations of the boundary. The other was embedded $2 \mathrm{~cm}$ at the top of the profile and was removed before fault displacement commenced. The vertical distance between the two instruments was $0.5575 \mathrm{~cm}$ in all cases. Both accelerometers were mounted to an acrylic foundation and protected from moisture using $100 \%$ silicone. A sample rate of $10000 \mathrm{~Hz}$ along with a signal input range of $-3 \mathrm{~g}$ to $3 \mathrm{~g}$ and a sensitivity set to 4046 $\mathrm{mVolts} / \mathrm{g}$ was used to record the acceleration data. Images of the accelerometers are shown in Figure 22.

(a)

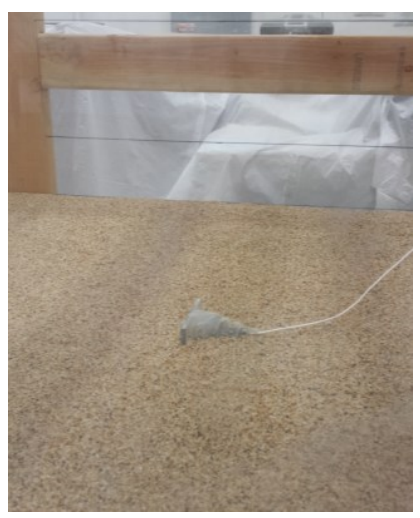

(b)

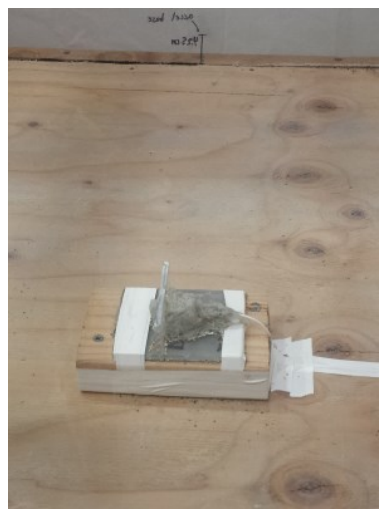

(c)

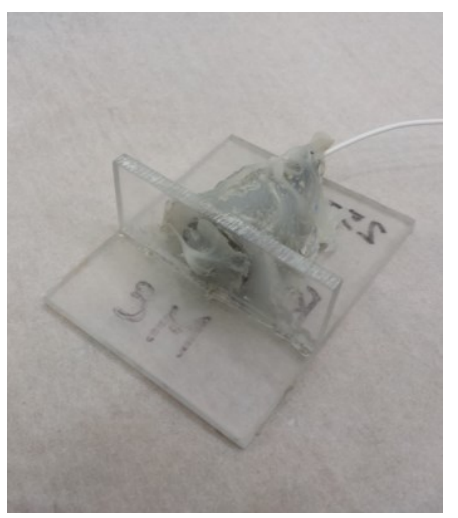

Figure 22: (a) Top accelerometer; (b) Base accelerometer; (c) Close-up of acrylic foundation and silicone protection.

\subsubsection{Displacement Data}

A wire strain potentiometer mounted to the base of the static section of the apparatus was connected to the moving end at a $45^{\circ}$ angle as shown in Figure 23. This configuration tracked the displacement during loading with respect to time. In addition, the data was 
used to verify that the velocity of the hanging wall during testing was approximately constant by taking the derivative of the displacement curve. Figure 24 through Figure 27 shows the displacement data taken for all tests. It should be noted that the virgin stiff clay displacement was halted after 11.5 seconds for approximately 35 seconds because the front end of the fault box had begun to lift off the ground and needed to be weighted down. This stoppage was edited out of Figure 26 for clarity.

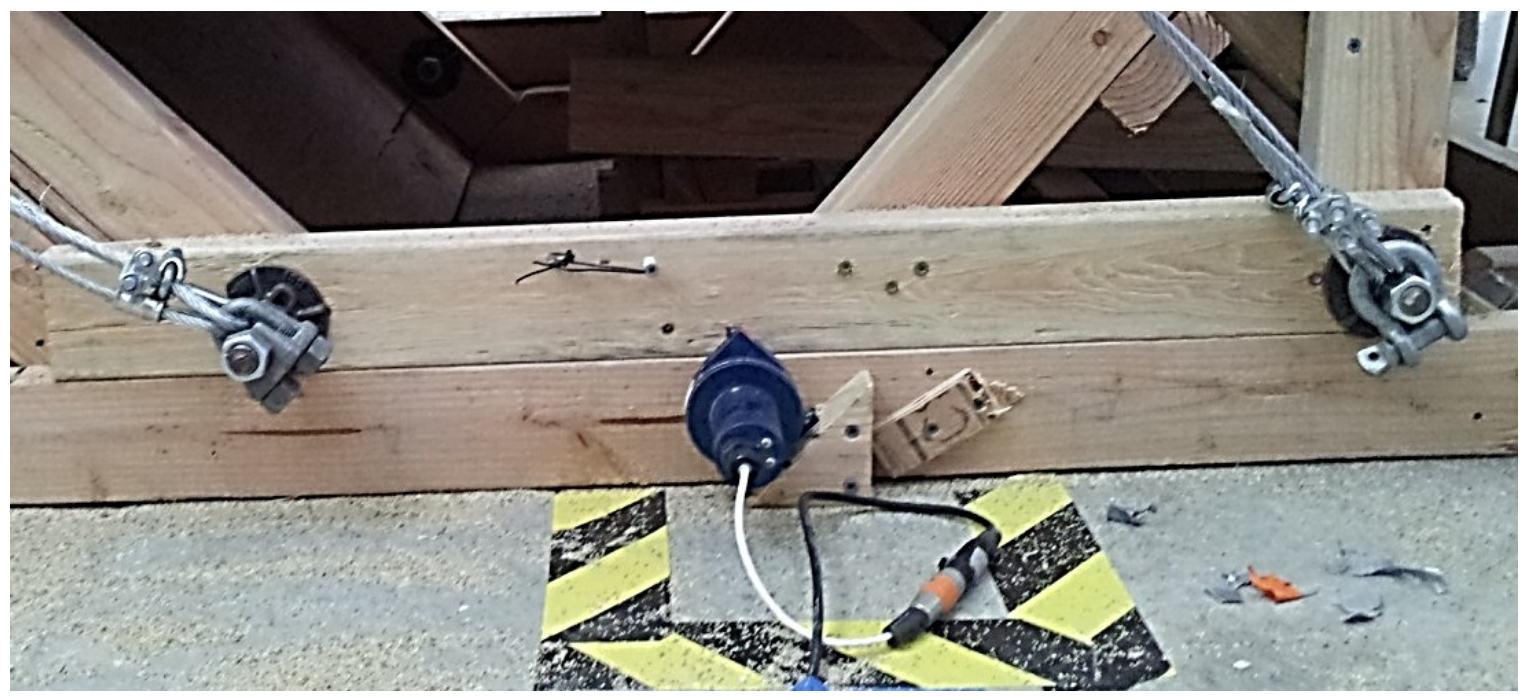

Figure 23: Wire strain potentiometer configuration.

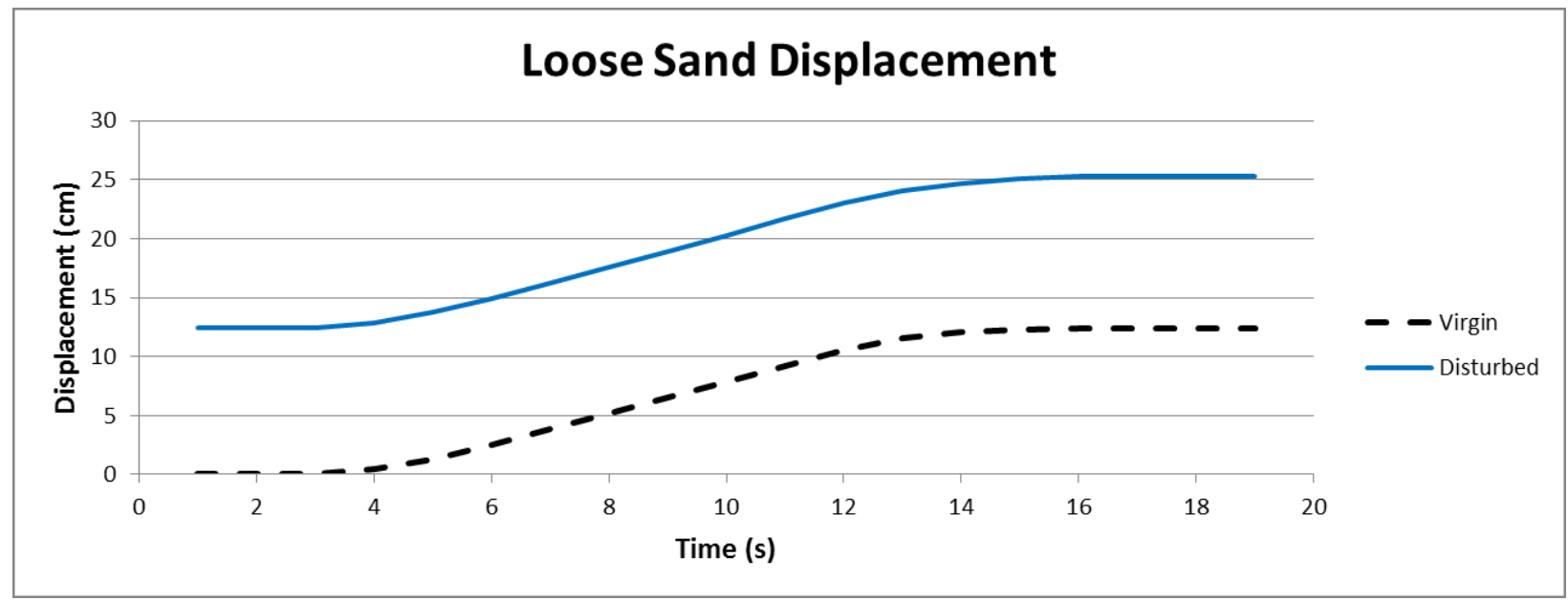

Figure 24: Displacement data collected during tests on loose sand. 


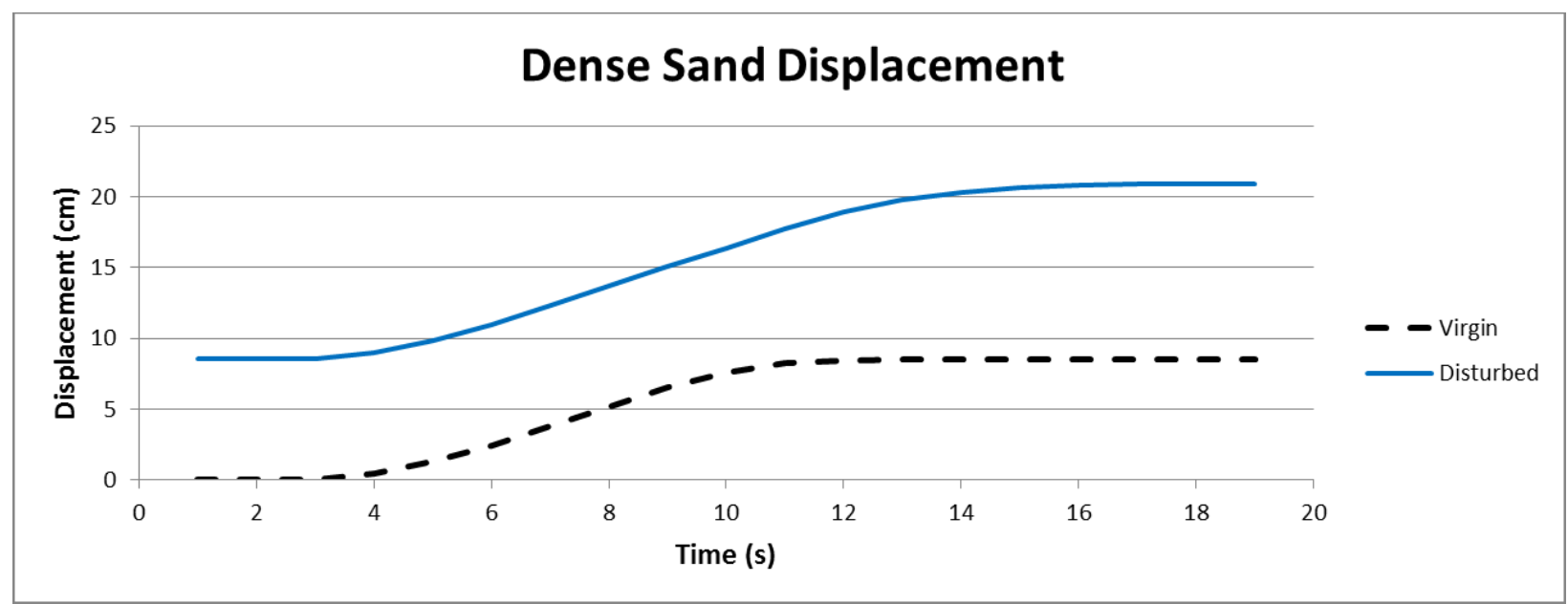

Figure 25: Displacement data collected during tests on dense sand.

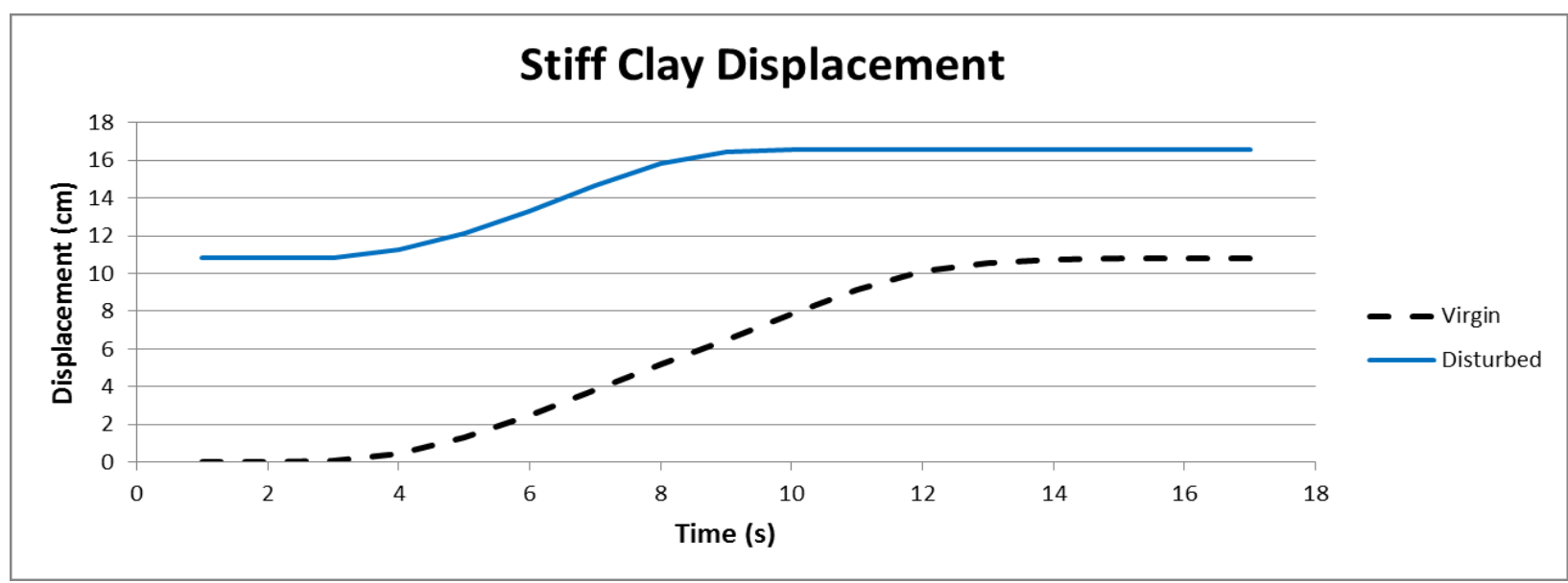

Figure 26: Displacement data collected during tests on stiff clay.

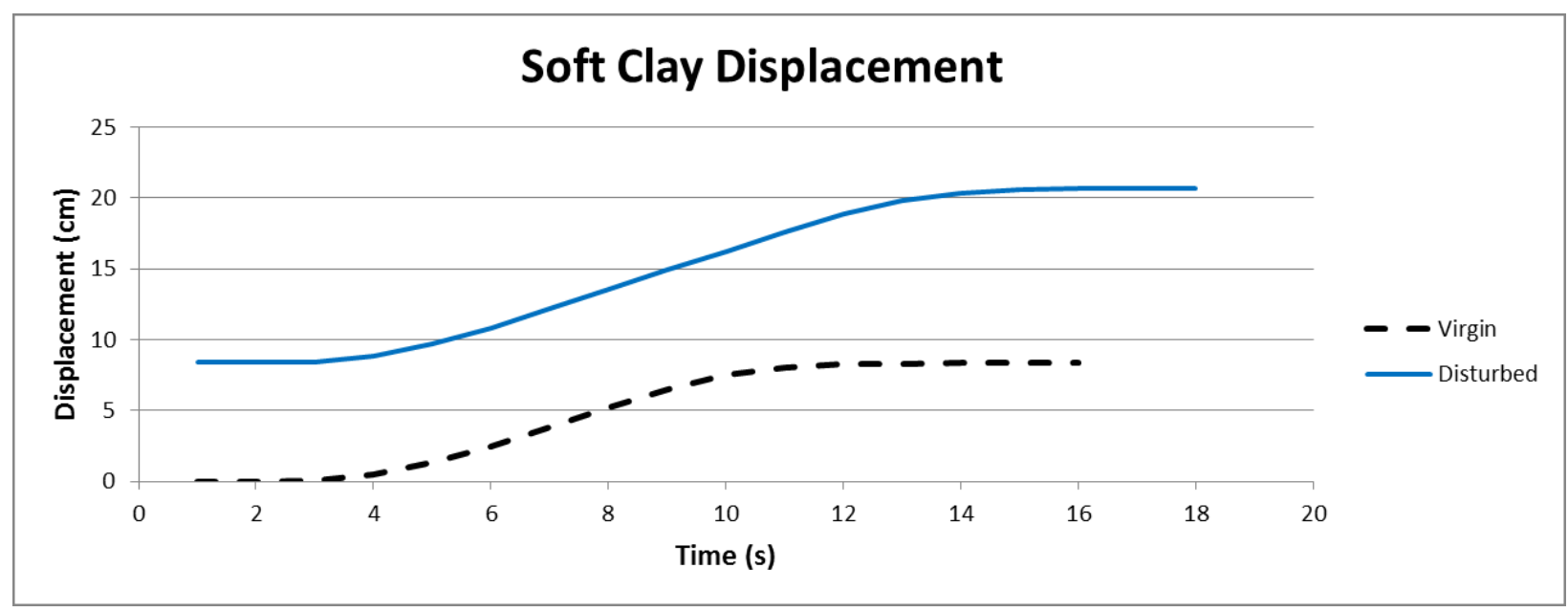

Figure 27: Displacement data collected during tests on soft clay. 


\subsubsection{Video Data}

Two SAMSUNG-SGH-I337 13 megapixel cameras captured the deformation of the entire soil profile from both sides. In addition, two Canon Rebel T2i cameras recorded a close-up view of the development of the shear band in each test. The quality of the images allowed for the precise time and displacement at which the shear band formed and surface rupture occurred to be determined after the fact.

To synchronize the video and displacement data, a camera was configured to record numeric displacement and time indicators created in LabView. The internal clock of this camera and all other cameras were synchronized by identifying their offsets using recordings independent from those taken during the fault box experiments and calibrating accordingly. This allowed the video records of the displacement indicator and the actual experiment to be temporally matched. As an added precaution, preceding the start of each test, a sound was generated by striking a hammer on steel once all recording devices were active but before shearing began. This served as reassurance that the recordings were synced with acceptable precision by providing an easily detected reference which was known to be temporally consistent across all of the recordings. Following testing, the video editing software Presto! Video Works 6 was used to format the video files to map the basal displacement and time. It was also used to ensure that the reference sound occurred at the same time in each video.

\subsection{Shear Wave Velocity}

The average shear wave velocity of each soil profile was obtained using a technique developed from trial and error. Through preliminary experiments in sand, bottom-up hammer blow tests were found to yield the most pronounced first arrival peaks needed for 
accurate waveform interpretation. Initially, a top down method was attempted as is in Crosariol (2009) but the results were much less favorable in both sand and clay than desired. Hence, multiple striking locations and methods were tested for a bottom up test which led to a working methodology that proved successful for both sand and clay.

Following the recommendations of Meymand (1998) and Crosariol (2009), blows were cushioned to generate lower frequency input. Thus, shear waves were generated by striking the front end of the box with a rubber mallet wrapped in cloth. The striking location was marked with padded tape and coincided with the elevation of the base accelerometer. Figure 28 shows the hammer used for delivering blows and the striking location on the box.

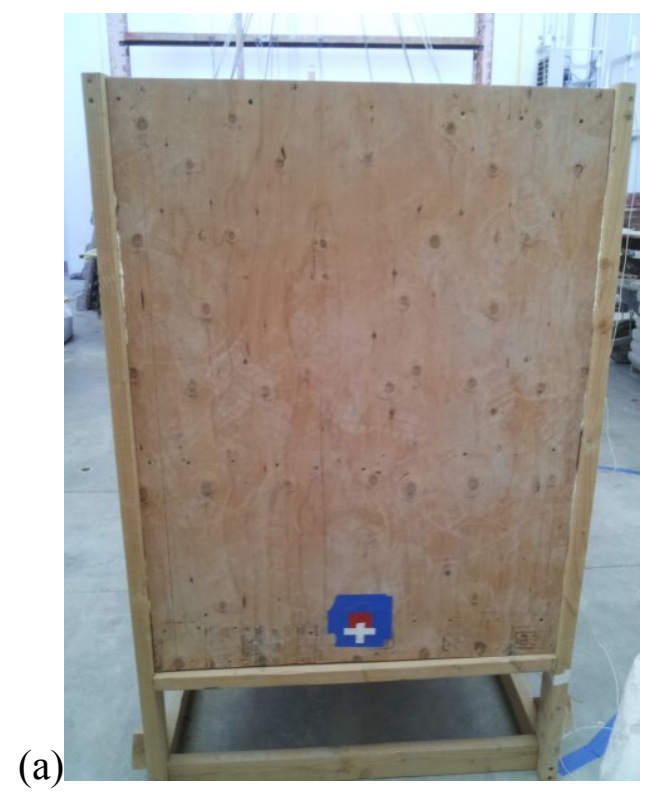

(b)

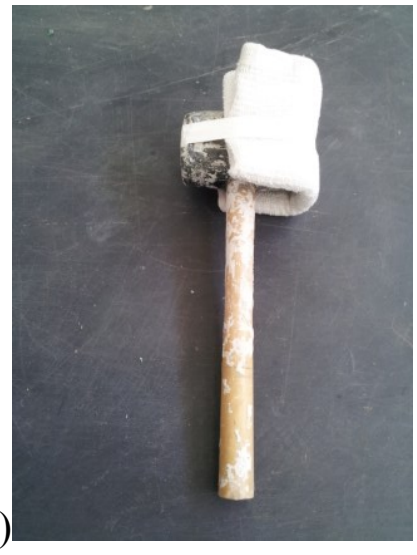

Figure 28: (a) Striking location for hammer blow tests marked with white cross; (b) hammer wrapped in towel to dampen blows

To ensure acceptable waveforms were recorded, the time differences between both the positive and negative first arrival peaks were determined. If the time differences were not 
nearly equal it was taken as an indication that the peak arrivals had not been identified correctly and test was thrown out. Figure 29 through Figure 32 show examples of typical waveforms generated for each material using the aforementioned methodology.

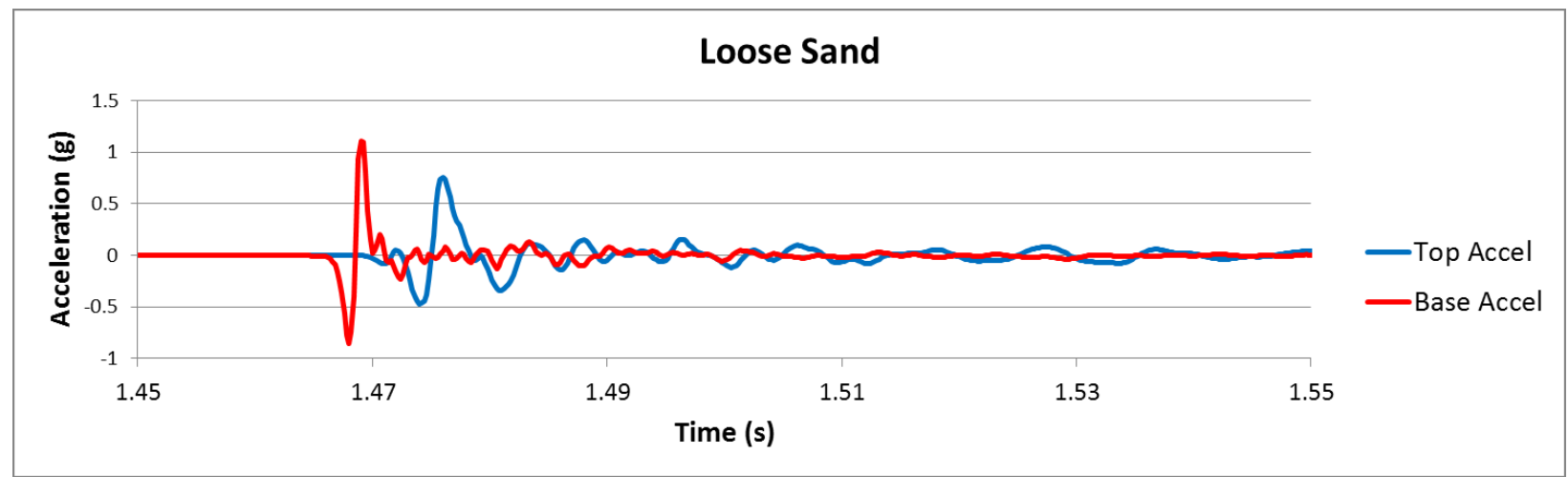

Figure 29: Example bottom up hammer blow test waveform used for shear wave velocity determination of loose sand.

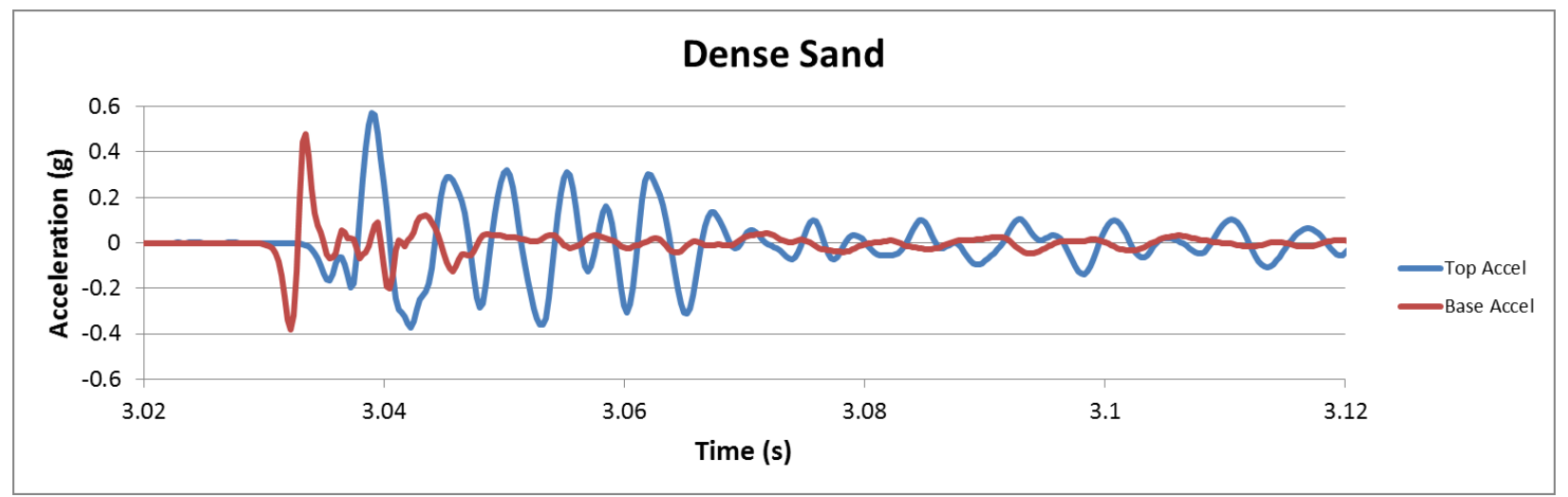

Figure 30: Example of bottom up hammer blow test waveform used for shear wave velocity determination of dense sand. 


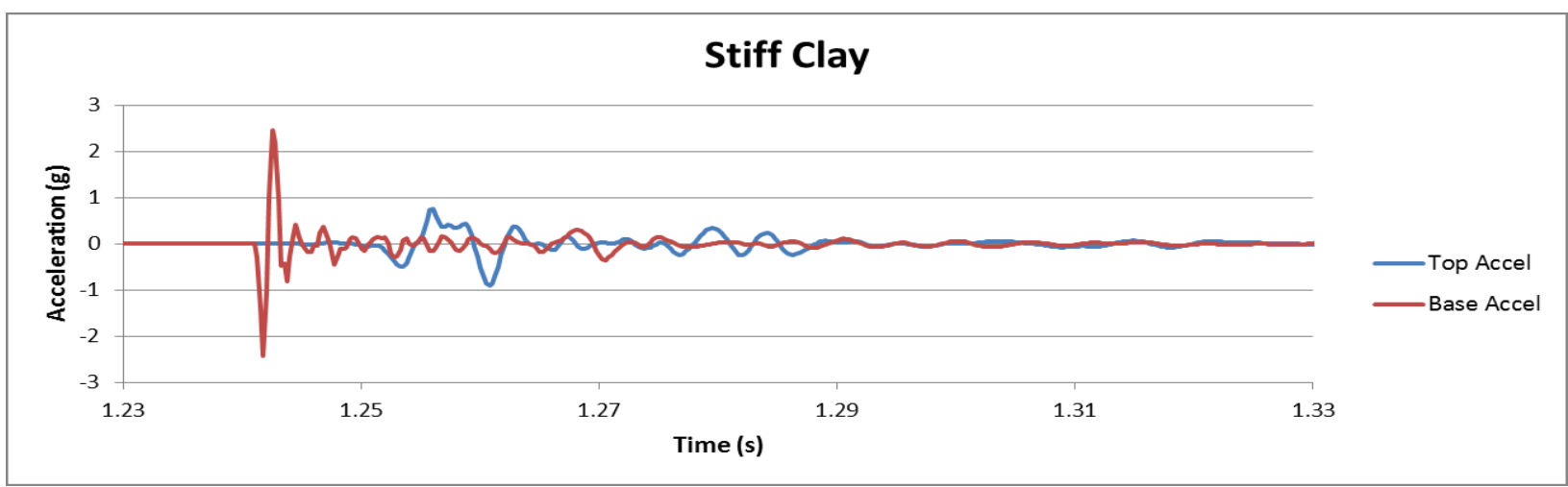

Figure 31: Example of bottom up hammer blow test waveform used for shear wave velocity determination of stiff clay.

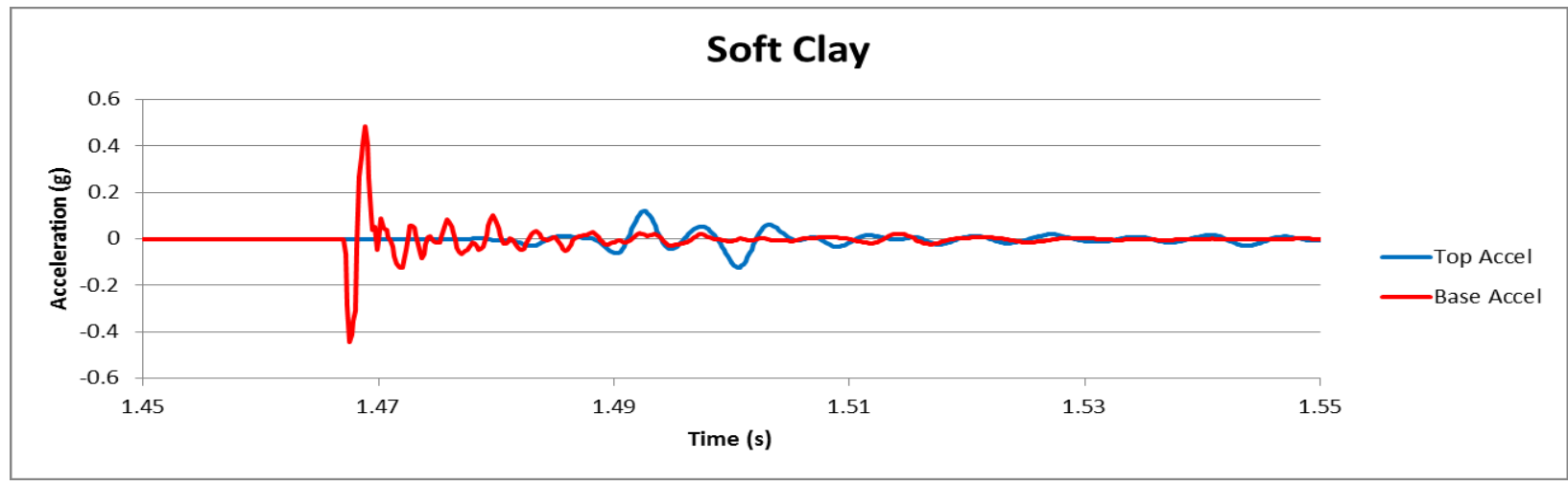

Figure 32: Example of bottom up hammer blow test waveform used for shear wave velocity determination of soft clay.

The acceleration time-histories revealed recurring trends as a function of the material. In general, the dense and loose sand waveforms appear much more susceptible to amplification than the other materials. This is indicated by the relatively large amplitude recorded by the top accelerometer compared to that of the base. To investigate whether or not this is reasonable, the discrete Fourier transform of each time-history was plot (Figure 33 through Figure 36) so that the dominant input frequencies generated by the hammer blows and recorded by the base accelerometer could be compared to estimated resonant frequencies of the soil profiles. The natural frequencies of the soil profiles can be estimated by inspection of the discrete Fourier transform plot of top accelerometer timehistory. The closer the two values the more amplification would be expected. 

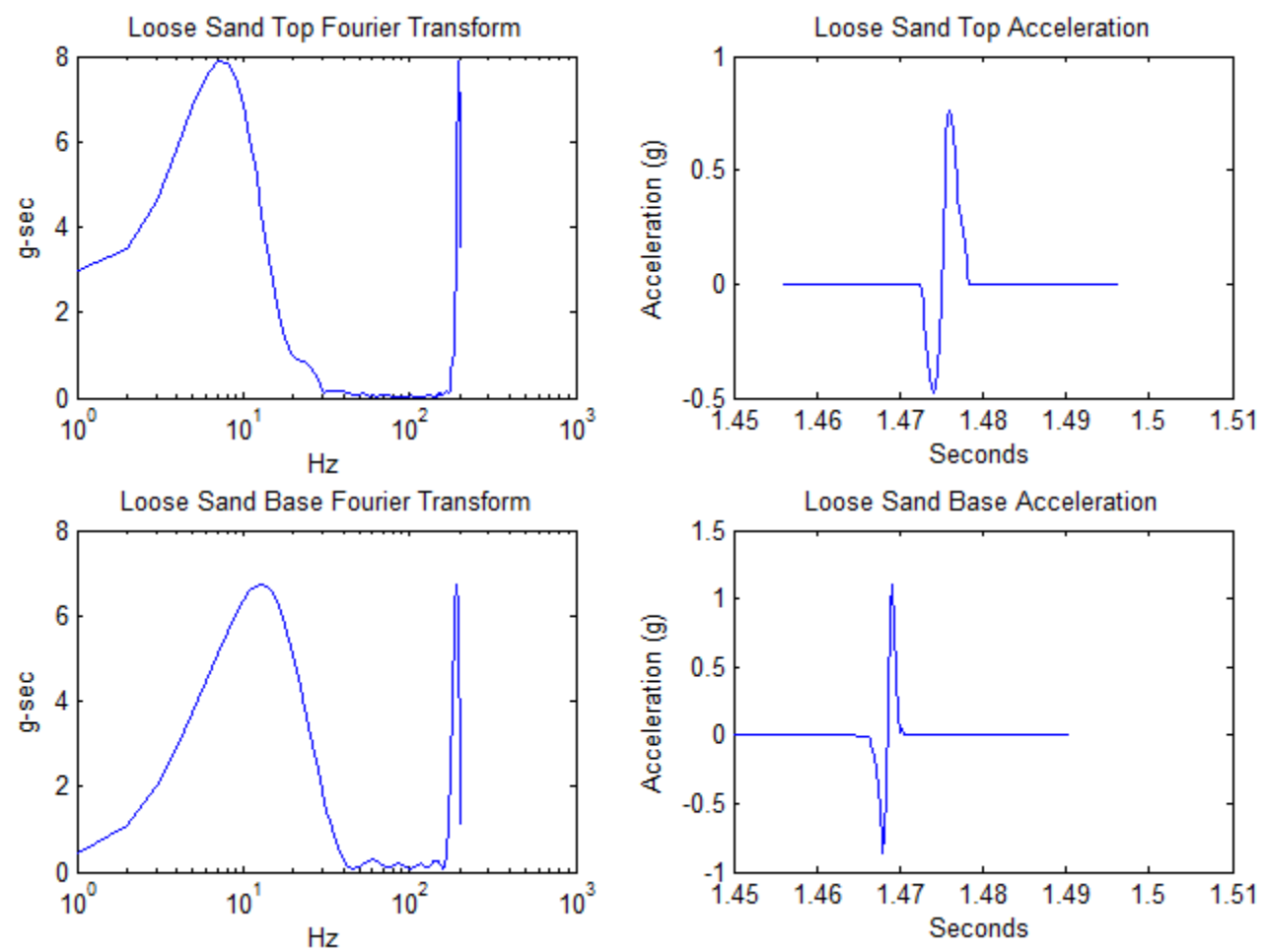

Figure 33: Acceleration time-history and discrete Fourier transform of accelerometer records in loose sand.
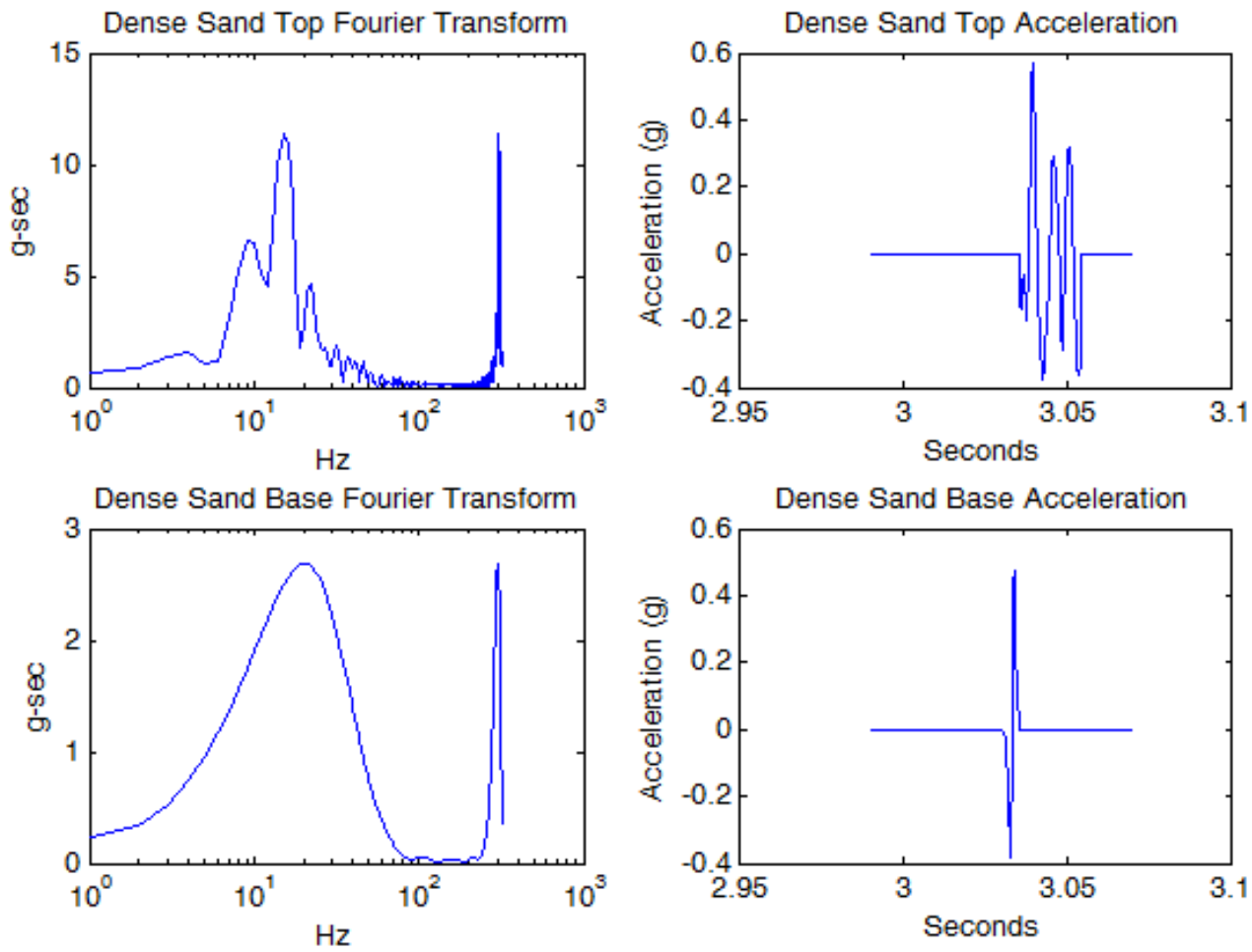

Figure 34: Acceleration time-history and discrete Fourier transform of accelerometer records in dense sand. 

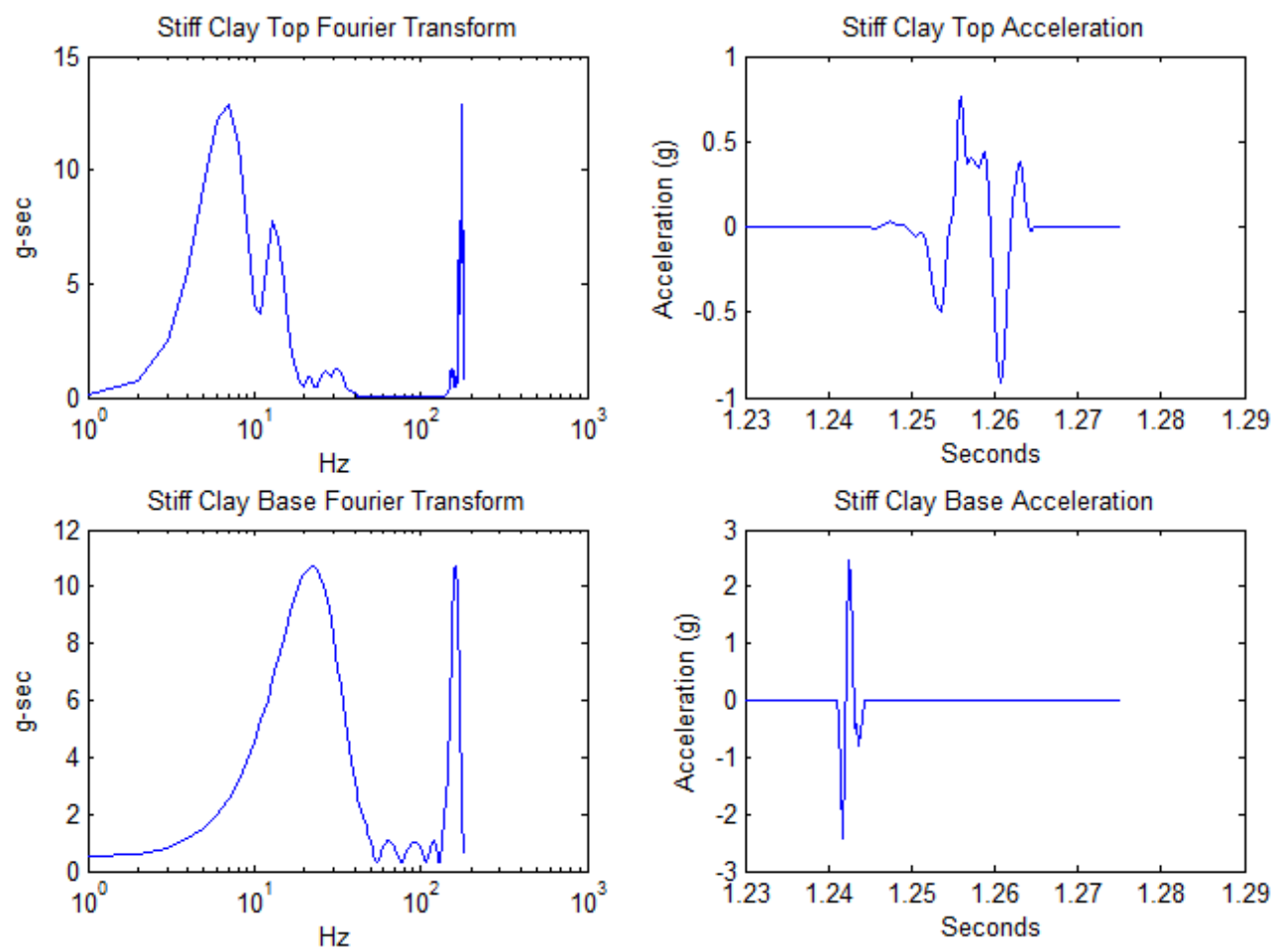

Figure 35: Acceleration time-history and discrete Fourier transform of accelerometer records in stiff clay.
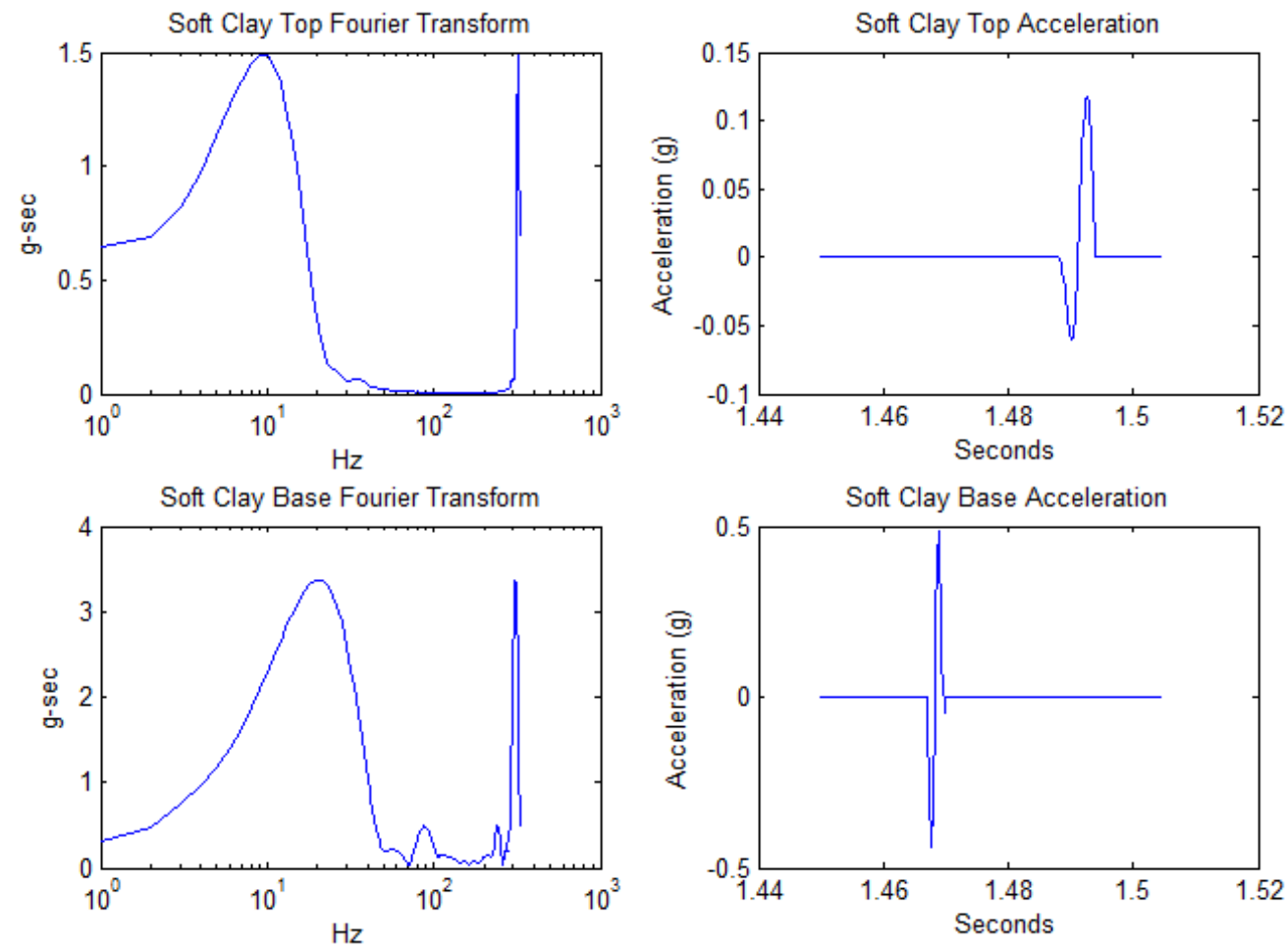

Figure 36: Acceleration time-history and discrete Fourier transform of accelerometer records in soft clay. 
Table 3: Dominant frequencies recorded by base (input) and top (surface) accelerometers.

\begin{tabular}{|c|c|c|}
\hline Material & $\begin{array}{c}\text { Dominant Input } \\
\text { Frequency }(\mathrm{Hz})\end{array}$ & $\begin{array}{c}\text { Dominant Surface Frequency } \\
(\mathrm{Hz})\end{array}$ \\
\hline Loose Sand & 12 & 7 \\
\hline Dense Sand & 20 & 15 \\
\hline Stiff Clay & 23 & 7 \\
\hline Soft Clay & 20 & 9 \\
\hline
\end{tabular}

Before generating the Fourier frequency domain plots, the waveforms were cropped so that appropriate frequency content was analyzed and effects of waves which travelled through the walls of the box did not influence the results. By doing this, we see that the frequency domain plots provide a possible explanation for the apparent amplification seen in the sand tests. The dominant input frequencies are much closer to those recorded by the top accelerometers than for the either the stiff or soft clay. Considering this evidence, it is reasonable that some of the time histories for dense and loose sand portray amplitudes at the surface similar and in some cases greater than those at the base.

It should be noted that the time-histories presented above document only one of 12 independent hammer blow tests conducted for each material. Although there was very little variation between the characteristics of each data set, there were some cases in which the calculated shear wave velocity differed slightly for a given material. Thus, the averages of all 12 tests for each material were used for the final shear wave velocity designations. Summary statistics of these tests are shown in Table 4 for each case analyzed. Equation 3 describes how each individual shear wave velocity designation was calculated. 


$$
V_{s}=\frac{t_{\text {surface }}-t_{\text {base }}}{d}
$$

In Equation 3, $t_{\text {surface }}$ is the time of the first arrival peak recorded by the top accelerometer, tbase is the time of the first arrival peak recorded by the base accelerometer and $d$ is the distance between the two instruments, taken as 0.576 meters in all cases.

Table 4: Average shear wave velocity and range of 12 hammer blow tests for each material

\begin{tabular}{|c|c|c|c|}
\hline Material & $\begin{array}{c}\text { Average Shear } \\
\text { Wave Velocity }(\mathbf{m} / \mathbf{s})\end{array}$ & Range (m/s) & $\begin{array}{c}\text { Coefficient of } \\
\text { Variation (\%) }\end{array}$ \\
\hline Loose Sand & 77.0 & $74.65-79.04$ & 1.91 \\
\hline Dense Sand & 101.4 & $96.96-106.19$ & 1.95 \\
\hline Soft Clay & 23.65 & $23.40-23.90$ & 0.71 \\
\hline Stiff Clay & 41.80 & $40.55-42.89$ & 1.44 \\
\hline
\end{tabular}




\section{$4 \quad$ Testing}

\subsection{Material Properties}

\subsubsection{Sand Properties}

The sand used for tests 1 and 2 was from the same batch of \#2/16 Lapis Lustre Sand from the CEMEX Lapis Plant near Monterey, California. The method used to control the density of the sand for testing is described in section 4.2. Figure 37 shows the approximate gradation curve for the sand as reported by CEMEX quality control.

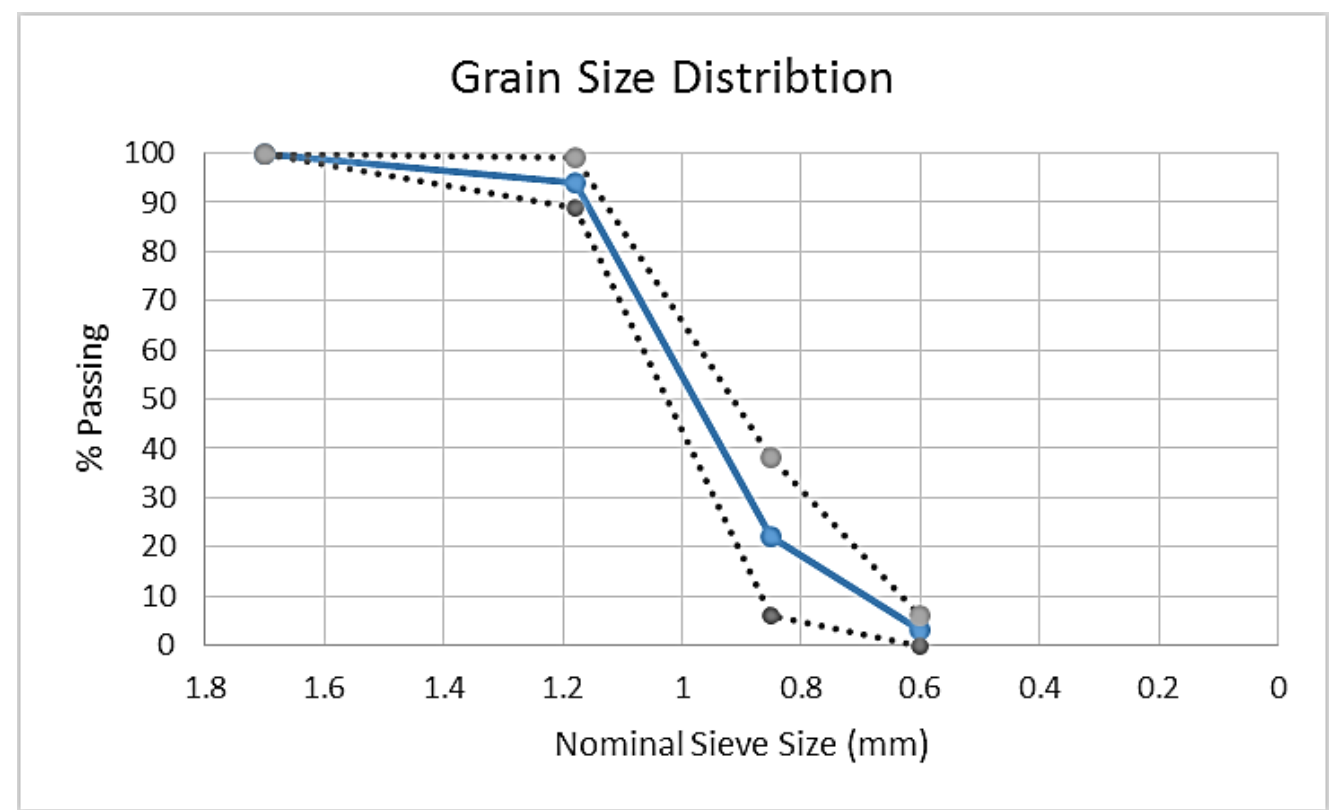

Figure 37: Grain size distribution curve for the \#2/16 Monterey Sand. The solid line is the estimated average and the dashed lines are the upper and lower bounds.

\subsubsection{Clay Properties}

The clay used in tests 3 and 4 was a scale model mixture of San Francisco Bay Mud and consisted of kaolinite, bentonite, class C fly ash, and water. Separate batches of clay were mixed approximately on year apart to give different water contents for the stiff and soft 
clay experiments. The bentonite used in the mix design was American Standard 200 mesh from Scott Sales Company in Huntington Park, CA; the Kaolinite was Kamin 35 from the PT Hutchins Company of City of Industry, CA; and the class C fly ash was from Mineral Resources Technologies which is a subsidiary of CEMEX USA. Table 5 describes the recipe used for both the stiff and soft clay. The recipe was developed based on solid proportions of $67.5 \%$ kaolinite, $22.5 \%$ bentonite and $5 \%$ class $\mathrm{C}$ fly ash as well as a target water content of $125 \%$. The mixture used for the soft clay was the same as in Crosariol (2009) when the stiff clay was originally mixed.

Table 5: Recipe used to develop model mixture of San Francisco Bay Mud.

\begin{tabular}{|c|c|}
\hline Ingredient & Percent of Total Mix by Weight \\
\hline Kaolinite & 30.13 \\
\hline Bentonite & 10.04 \\
\hline Class C Fly Ash & 4.28 \\
\hline Water & 55.56 \\
\hline
\end{tabular}

While loading the box with clay, two samples from each layer were taken and their water contents measured to provide insight as to how much of an affect one year's time had on the index properties of the stiff clay. The results are compared in Table 6. In addition, a torvane shear tester was used to estimate the undrained shear strength of both clays as the box was filled for tests 3 and 4 . The results, presented in Table 7, represent the average strength estimate for each layer before adding overburden material and are based on multiple measurements in each layer. 
Table 6: Water content data for the stiff and soft clay mixtures close to the time of testing.

\begin{tabular}{|c|c|c|}
\hline & \multicolumn{2}{|c|}{ Average Water Content (\%) } \\
\hline Sample Depth (cm) & Stiff Clay & Soft Clay \\
\hline 55 & 67.7 & 114.0 \\
\hline 45 & 70.1 & 113.3 \\
\hline 35 & 72.8 & 115.8 \\
\hline 25 & 77.8 & 122.8 \\
\hline 15 & 74.3 & 118.9 \\
\hline 5 & 81.4 & 117.9 \\
\hline Average & $\mathbf{7 3 . 9}$ & $\mathbf{1 1 7 . 1}$ \\
\hline
\end{tabular}

Table 7: Average undrained shear strengths of each layer in stiff and soft clay.

\begin{tabular}{|c|c|c|}
\hline & \multicolumn{2}{|c|}{ Average Undrained Shear Strength (kPa) } \\
\hline Mean Layer Depth (cm) & Stiff Clay & Soft Clay \\
\hline 55 & 4.10 & 1.20 \\
\hline 45 & 3.83 & 1.00 \\
\hline 35 & 3.87 & 0.93 \\
\hline 25 & 5.10 & 1.45 \\
\hline 15 & 3.43 & 1.21 \\
\hline 5 & 3.27 & 1.32 \\
\hline Average & 3.93 & 1.19 \\
\hline
\end{tabular}

The average undrained shear strength was correlated to the shear wave velocity using Equation 4 from Dickenson (1994). This provided an indication of what values would be reasonable when determining the shear wave velocity directly.

$$
V_{s}=18\left(S_{u}\right)^{0.475}
$$

In Equation $4, V_{s}$ is shear wave velocity in feet per second (fps) and $S_{u}$ is the undrained shear strength in pounds per square foot (psf). Based on the average undrained strengths of given in Table 7, the expected shear wave velocities for the stiff and soft clays are 44.5 $\mathrm{m} / \mathrm{s}$ and $25.3 \mathrm{~m} / \mathrm{s}$, respectively. These values agree very closely with the values for shear wave velocity from Table 4 which were determined directly. 


\subsection{Test Preparation}

\subsubsection{Placement of Sand}

Loose sand was loaded in approximately $2 \mathrm{~cm}$ flights using a perforated sack suspended from the crane (Figure 38). The sand was pluviated through the sack as the crane moved slowly across the loading area at a constant height of 1 meter above the initial soil surface during each pour. A broom was used to level the surface between each pour and marker beds were placed at $10 \mathrm{~cm}$ increments.

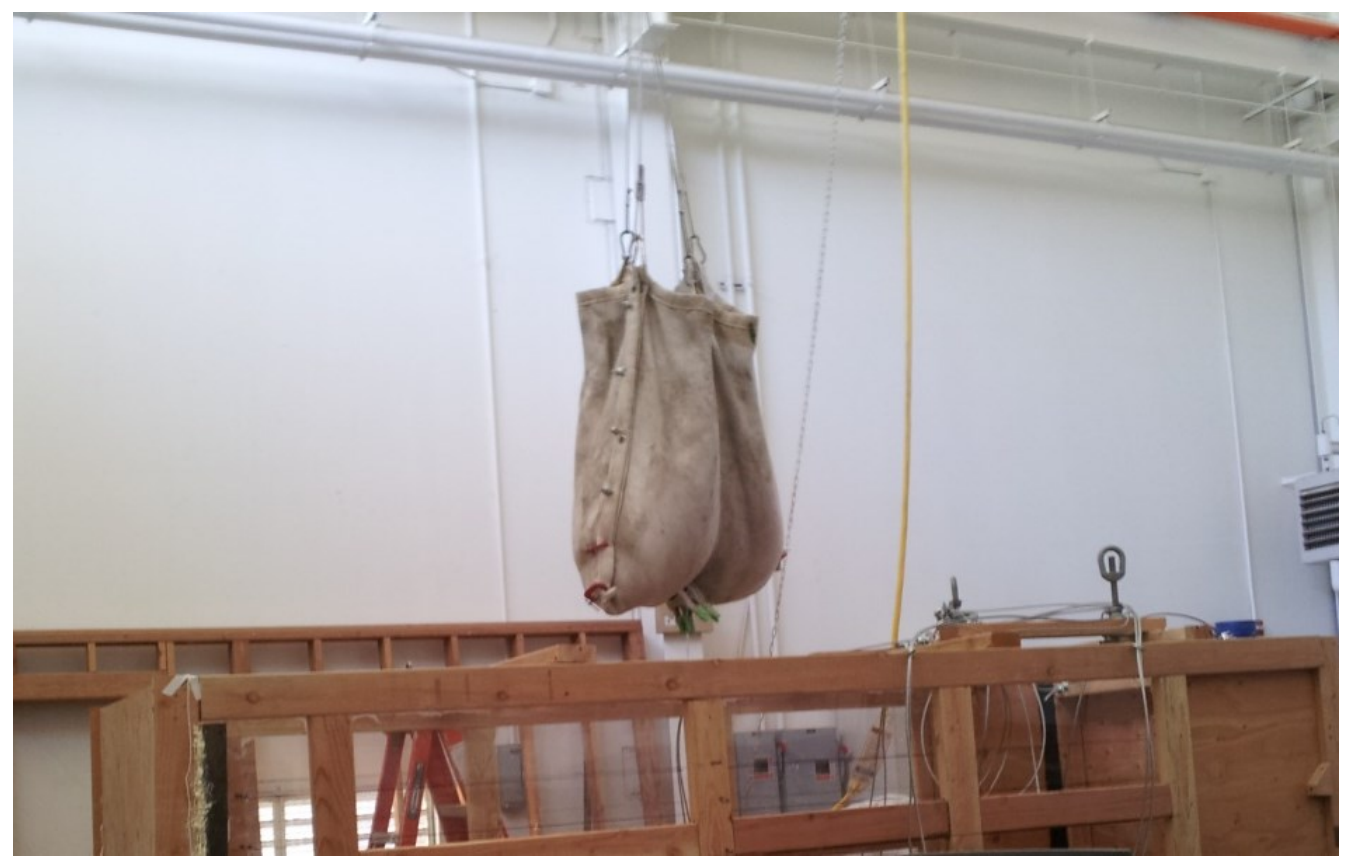

Figure 38: Perforated sack used to pluviate sand.

Chalkline was used to create the marker beds in both the dense and the loose sand. It should be noted that an additional marker bed was placed $2 \mathrm{~cm}$ from the surface in the dense sand profile. Although it is possible to interpret the results without this layer, it is much more difficult. 
Dense sand was loaded in exactly the same manner as loose sand except that sand was vibrated after placing of each marker bed. Layers were placed in $6 \mathrm{~cm}$ lifts and vibrated until the topmost lift had settled to $5 \mathrm{~cm}$. To induce vibrations, plywood was placed on the top of the profile and weighted down with steel which also served as striking locations for horizontal hammer blows delivered from inside the box. Simultaneously, the walls of the apparatus were struck with rubber mallets at nearly the same frequency, approximately $5 \mathrm{hz}$, to aid the densification process. To ensure a reasonably consistent shear wave velocity profile, rough measurements were taken after each layer was completed. If a layer settled the specified $1 \mathrm{~cm}$ but the measured shear wave velocity after the lift was determined to be lower than the previous, densification continued while additional sand was added to maintain the desired layer thickness.

\subsubsection{Placement of Clay}

The stiff clay was loaded into the box via large buckets attached to the ceiling crane. After each bucket was filled by hand, the crane lifted it in into the fault box where it was emptied and its contents spread out evenly. Alternatively, the soft clay was delivered to the fault box straight from the ChemGrout mixer (Figure 39), which was used to prepare the soft clay immediately before testing. The stiff clay was also prepared by the same ChemGrout mixer but it had been in storage for approximately one year. A schematic of the ChemGrout mixer is shown in Figure 40 and a detailed procedure for using it to achieve the target mix from this study is given in Crosariol (2009). 


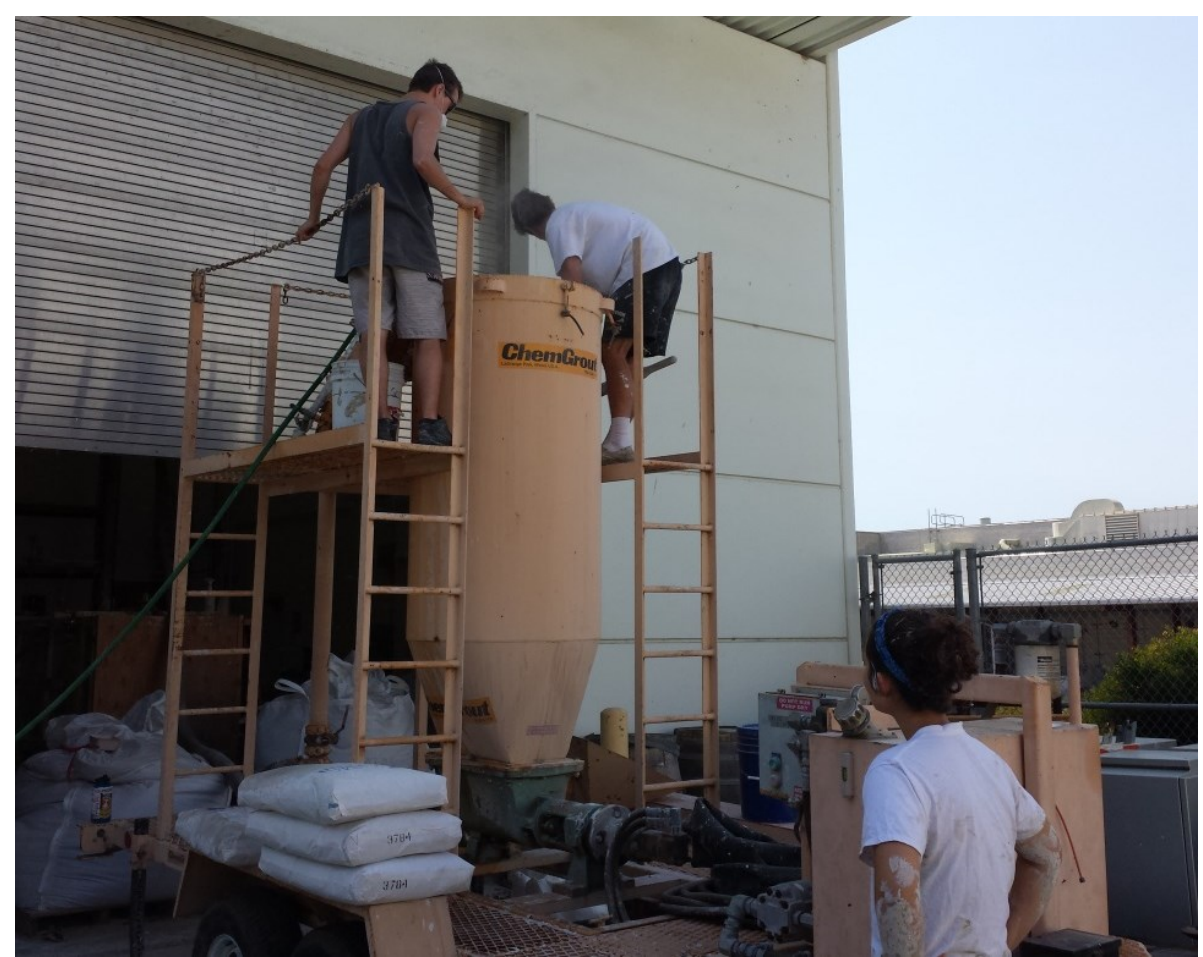

Figure 39: Mixing soft clay with the ChemGrout mixer.

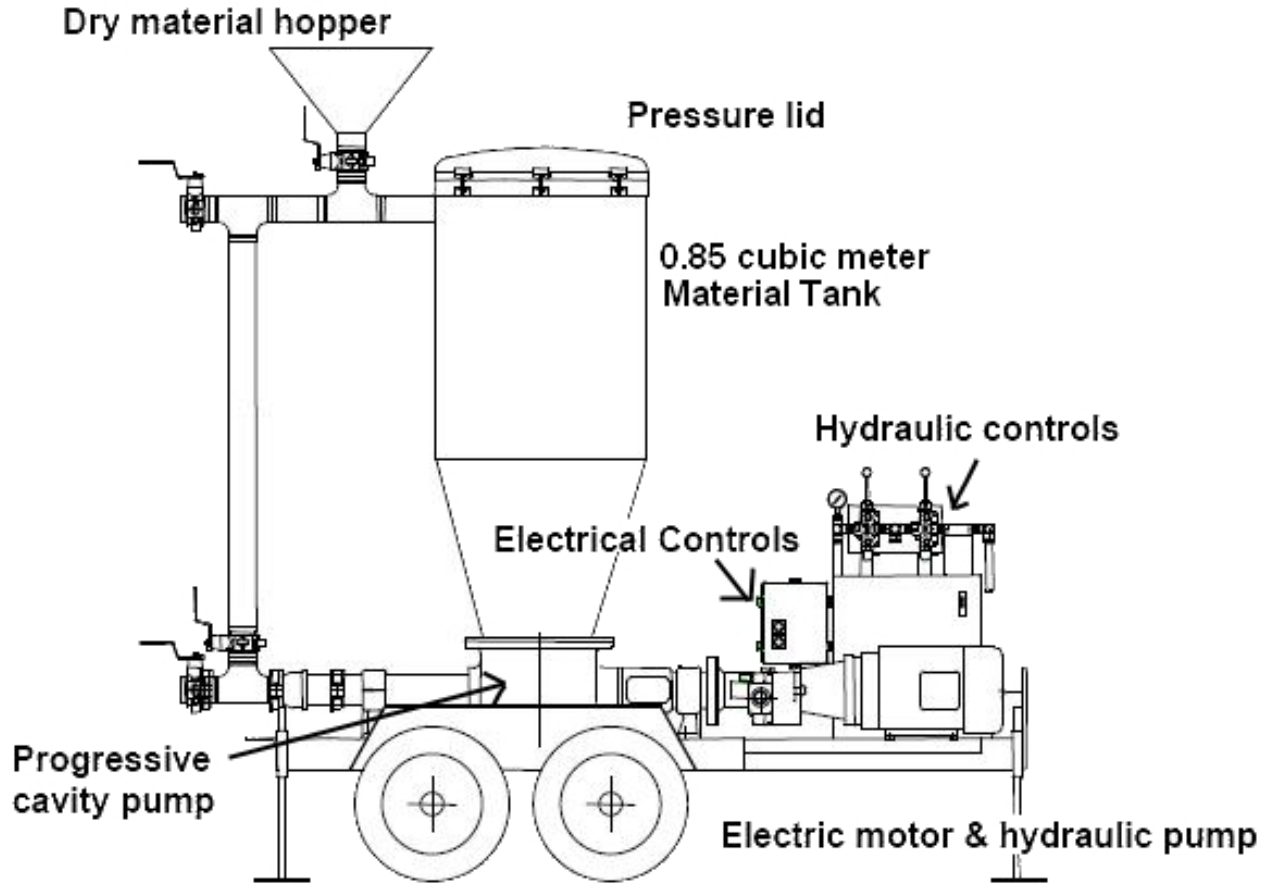

Figure 40: From Meymand (1998). Schematic of the ChemGrout mixer. 
A trial and error process was invoked to determine the most efficient method to load the clay without creating voids or inconsistent compaction. As a result, a working methodology for filling the fault box with clay was developed and is outlined below:

1) All at once, load a volume of clay equal to one $10 \mathrm{~cm}$ lift into the fault box, concentrating most of the volume into the center and away from the walls. For the stiff clay, deliver the clay using buckets attached to the ceiling crane. The soft clay is delivered directly from the ChemGrout mixer.

2) Situate two persons outside the container to watch for voids against the Plexiglass walls and an additional two inside to spread the clay. In the soft clay, it is useful for those inside the fault box to spread out their weight over plywood boards to avoid sinking.

3) Remove clay in small portions from the central volume and carefully press it into the edges, corners, and against the walls. Listen to cues from those standing outside the apparatus to prevent unnoticed voids from going unaddressed.

4) Slowly form the lift up to a uniform height from the walls inward, continually leveling with painting spackles and demonstrated in Figure 41.

5) Upon the completion of each $10 \mathrm{~cm}$ interval, lay the thin elastic strips such that one half of the width is pressed into the clay, normal to the wall, and the other is flush against the wall. This will result in a right angle along the entire strip.

6) Repeat steps 1 through 5 until the desired depth of the soil profile has been achieved. No marker bed is necessary at the surface. 


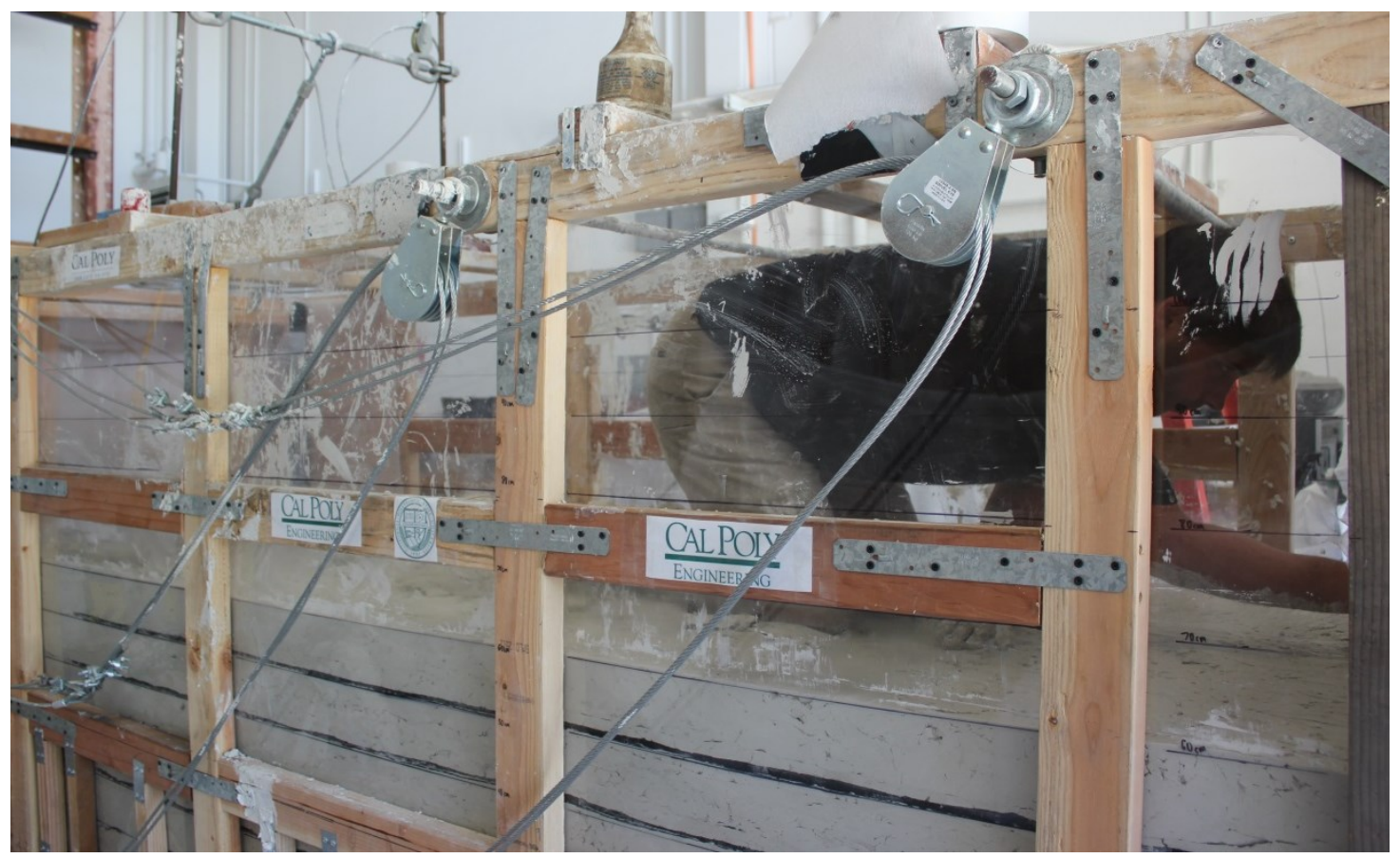

Figure 41: Spreading out clay from the walls inward and leveling with a painting spackle.

At each ten centimeter interval, $2 \mathrm{~cm}$ wide thin black elastic strips were placed at the edges to act as marker beds. The elastic strips were folded at a right angle with half protruding into the clay and the other half pressed against the Plexigless. Sample images of the elastic strips are shown in Figure 42.

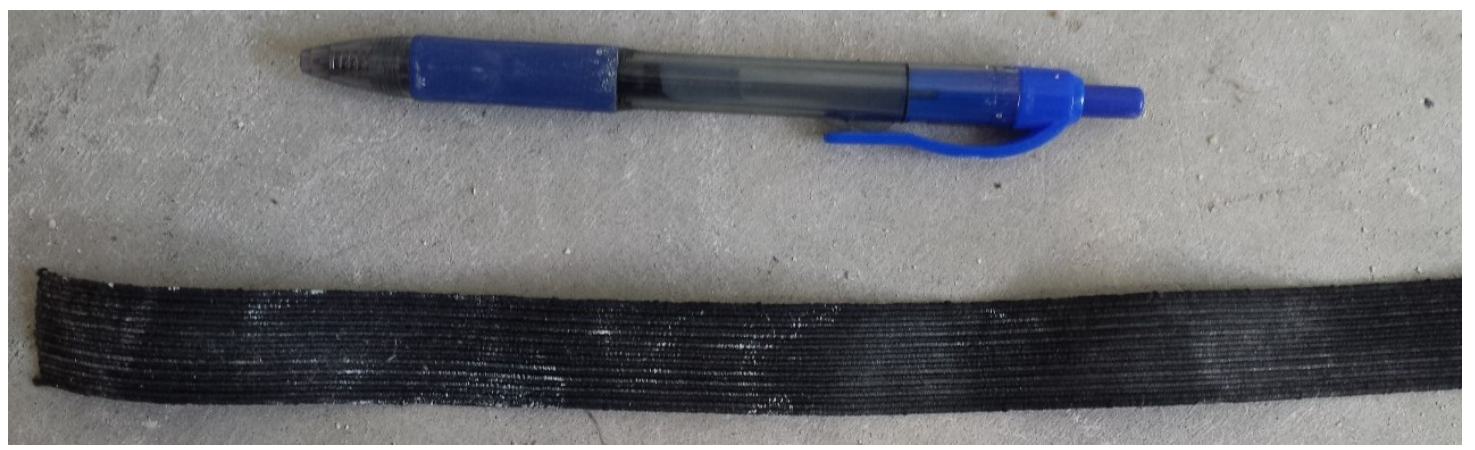

(a)

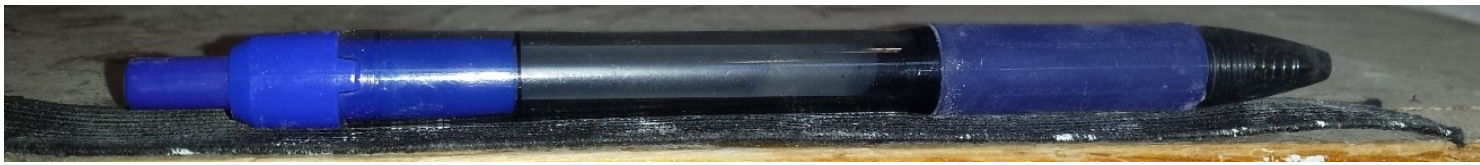

Figure 42: $2 \mathrm{~cm}$ wide elastic strips used to create marker beds in the clay. (a) Top view; (b) Side view. 


\subsection{Test Execution}

Testing consisted of two phases. In phase 1, rupture was initiated in virgin soil and continued until a clearly developed shear band reached the surface. At that point, movement of the hanging wall stopped and the total basal displacement was recorded. It should be noted that during testing the time at which the shear band reached the soil surface could only be roughly estimated. The exact time and corresponding basal displacement at which localized soil deformation began to control (after shear band formation) was determined from review of the video data. The step by step procedure carried out during phase 1 is given below.

\section{Phase 1 Procedure}

1) Turn on all video recording devices. There were two cameras on each side of the fault box and one recording the numeric displacement indicator.

2) Begin collecting displacement data in LabView.

3) Strike steel plate with a hammer to provide a means of checking that the video data is properly synchronized after the fact.

4) Turn on 3 ton ceiling crane and drive fault displacement until surface rupture is evident from both sides of the fault box.

5) Shut off recording devices halt data acquisition. 
In phase 2, the disturbed material was subject to further displacement equal to or exceeding the total amount of basal displacement from phase 1 . The surface of the soil was smoothed with either a broom (sand) or a painting spackle (clay) and between phases to allow a new scarp to form if possible. This allowed the time and displacement at which subsequent surface rupture occurred to be more clearly defined. The step by step procedure carried out during phase 2 is as follows:

\section{Phase 2 Procedure}

1) Smooth over the scarp developed in phase 1 using either a broom (sand) or a painting spackle (clay). Do this without disturbing material below a $3 \mathrm{~cm}$ depth (i.e. do not enter the fault box).

2) Turn on all video recording devices. There were two cameras on each side of the fault box and one recording the numeric displacement indicator.

3) Begin collecting displacement data in LabView.

4) Strike steel plate with a hammer to provide a means of checking that the video data is properly synchronized after the fact.

5) Turn on 3 ton ceiling crane and drive fault displacement until surface rupture is evident from both sides of the fault box and the basal displacement reaches a value equal to or greater than that of phase 1 .

6) Shut off recording devices halt data acquisition. 


\section{$5 \quad$ Results}

\subsection{Data Analysis}

The displacement required to generate surface rupture in a given run is unique to an experiment of precisely this scale. Thus, it is desirable to introduce a normalized measure

of a soil's susceptibility to surface rupture given its characteristic shear wave velocity and state (virgin/disturbed). With this in mind, it seems more telling to normalize the total basal displacement by the depth of the soil profile. This allows these results to be interpreted alongside those of other studies with greater ease.

Oettle and Bray (2013) concluded that soil rupture processes consist of two distinct phases: broad deformation followed by strain localization (shear band formation). Hence, for the purpose of this study, surface rupture was defined as the point at which further displacement at the base was entirely accommodated by localized strain in the soil instead of broad deformation. Table 8 shows the influence of various parameters on the total displacement required for surface rupture and the corresponding normalized vertical base displacement $h / H$, where $h$ is the vertical displacement required for surface rupture and $H$ is the height of the soil profile. This information is also portrayed graphically in Figure 43. 
Table 8: Basal displacement required for surface rupture given material type, relative stiffness, state and shear wave velocity

\begin{tabular}{|c|c|c|c|c|c|c|}
\hline Run & $\begin{array}{c}\text { Material } \\
\text { Type }\end{array}$ & $\begin{array}{c}\text { Relative } \\
\text { Stiffness }\end{array}$ & $\begin{array}{c}\text { Material } \\
\text { State }\end{array}$ & $\begin{array}{c}\text { Shear Wave } \\
\text { Velocity } \\
(\mathbf{m} / \mathbf{s})\end{array}$ & $\begin{array}{c}\text { Basal Displacement } \\
\text { Required for Surface } \\
\text { Rupture (cm) }\end{array}$ & $\boldsymbol{h} / \boldsymbol{H}$ \\
\hline 1.0 & Sand & Loose & Virgin & 77.0 & 12.44 & 0.147 \\
\hline 1.1 & Sand & Loose & Disturbed & 77.0 & 2.19 & 0.025 \\
\hline 2.0 & Sand & Dense & Virgin & 101.4 & 4.01 & 0.047 \\
\hline 2.1 & Sand & Dense & Disturbed & 101.4 & 0.50 & 0.0059 \\
\hline 3.0 & Clay & Stiff & Virgin & 41.8 & 5.73 & 0.068 \\
\hline 3.1 & Clay & Stiff & Disturbed & 41.8 & 1.15 & 0.014 \\
\hline 4.0 & Clay & Soft & Virgin & 23.65 & 8.37 & 0.097 \\
\hline 4.1 & Clay & Soft & Disturbed & 23.65 & 3.76 & 0.044 \\
\hline
\end{tabular}

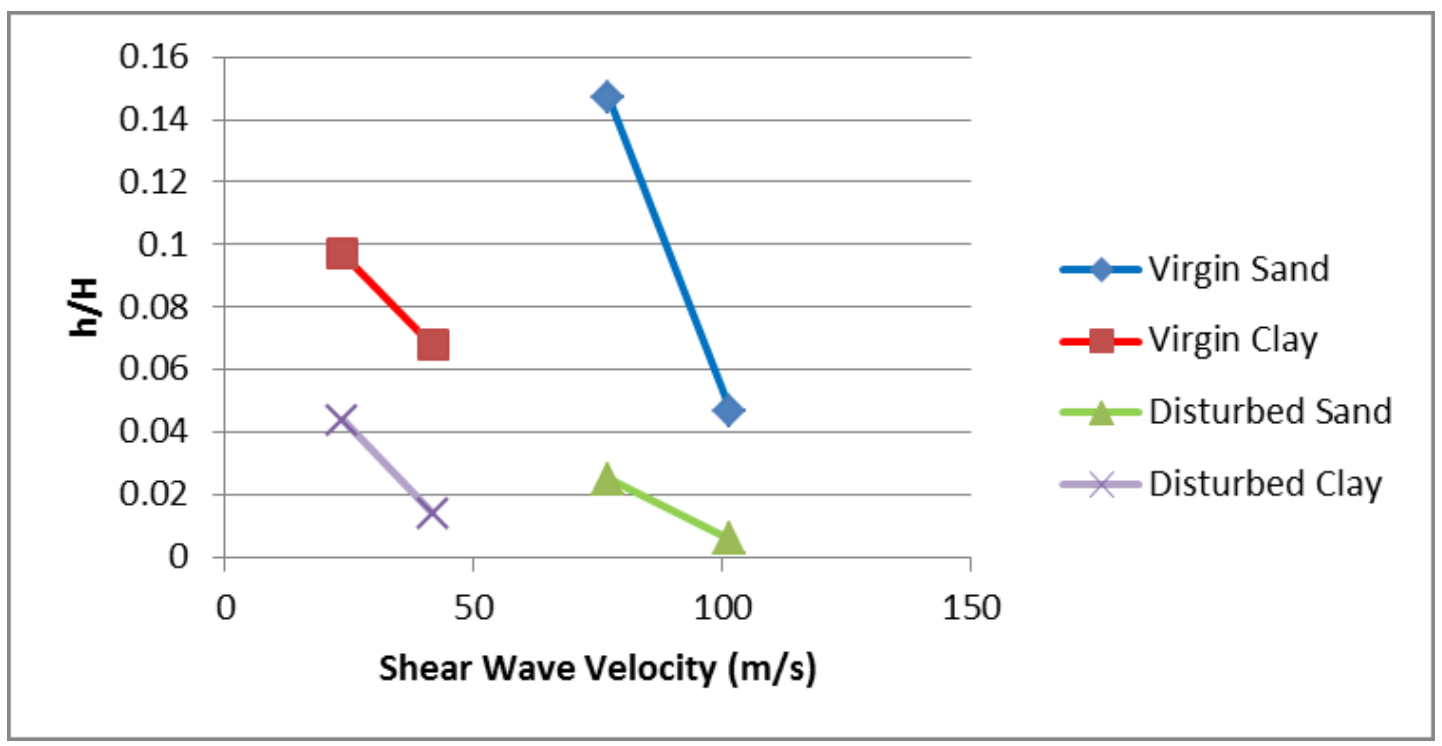

Figure 43: The relationship between shear wave velocity and normalized vertical displacement $(h / H)$ required for surface rupture in each material tested.

In Table 8, the relative stiffness parameter refers to the stiffness compared to soil of the same type. Alternatively, shear wave velocity could be thought of as a proxy for absolute stiffness because it is independent of material type. The material state describes whether or not the soil had been previously ruptured. For instances in which the material state is disturbed, the basal displacement required for surface rupture is measured from end of the previous test. Thus, for example, the total basal displacement at the end of run 1.1 is 
equal to the sum of the quantities for basal displacement for both runs 1.1 and 1.0 in Table 8.

A lower value of the normalized basal displacement, $h / H$, corresponds to more favorable conditions for surface rupture. Hence, Figure 43 indicates that increasing shear wave velocity promotes surface rupture for all materials tested. The normalized basal displacement, however, does not provide reliable insight as to exactly how much of an influence material stiffness has. This is because testing soils with a larger contrast in shear wave velocity would likely result in greater differences in normalized basal displacement. For example, we observed an average increase of $h / H$ by $132 \%$ when loose/soft material was tested as opposed to dense/stiff. If the average difference in shear wave velocity of the two material types tested were greater, the value of $h / H$ would likely be greater as well. Therefore, the results herein are only pertinent to the specific range of shear wave velocities from this study. More data would be needed to draw generalized conclusions.

Similar logic applies to make comparisons across different material types and rupture histories. The sand and clay in these experiments were of different average characteristic shear wave velocities which renders the comparisons made between the two materials only informative for making hypotheses. Regarding rupture history, it must be noted that these results could be different if collected for materials within a greater shear wave velocity range. 
The results presented in Table 8 tell us the following:

1) Even when the average shear wave velocity of sand is 2.7 times greater than that for sand, the normalized basal displacement, $(h / H)$, required for surface rupture is increased by an average of $40 \%$ when sand was tested compared to clay.

2) For an average shear wave velocity difference of $21.3 \mathrm{~m} / \mathrm{s}$, the normalized basal displacement required for surface rupture is increased by an average of $132 \%$ when loose or soft material was tested as compared to dense or stiff material.

3) For the specific materials in this study, the normalized basal displacement required for surface rupture is increased by an average of 304\% when virgin soil was tested as compared to disturbed soil.

Figure 44 through Figure 51 consists of select images from each of the tests performed. 


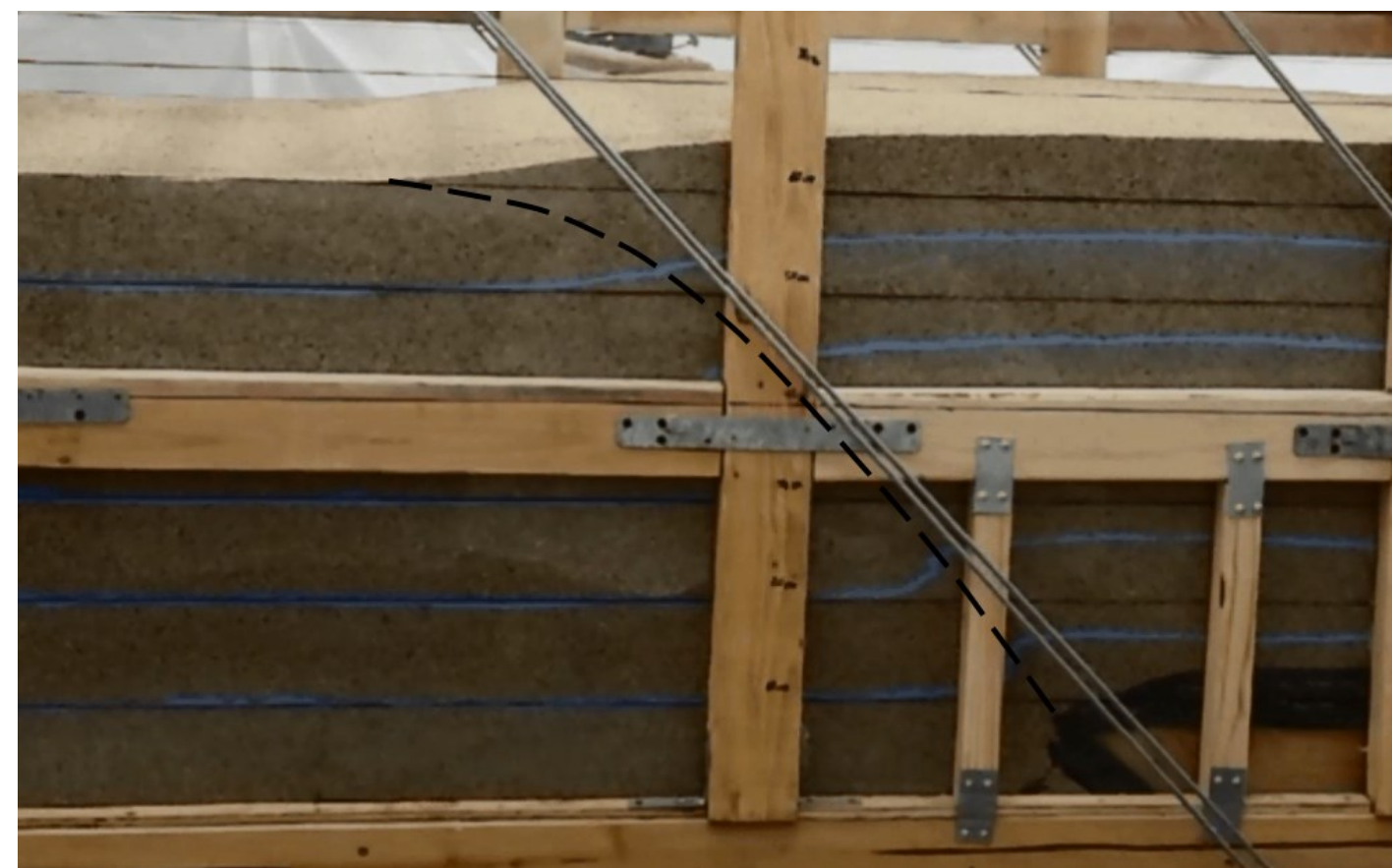

Figure 44: $12.44 \mathrm{~cm}$ of basal displacement beneath loose virgin sand. The dotted line represents the rupture plane. The edges have been cropped for clarity.

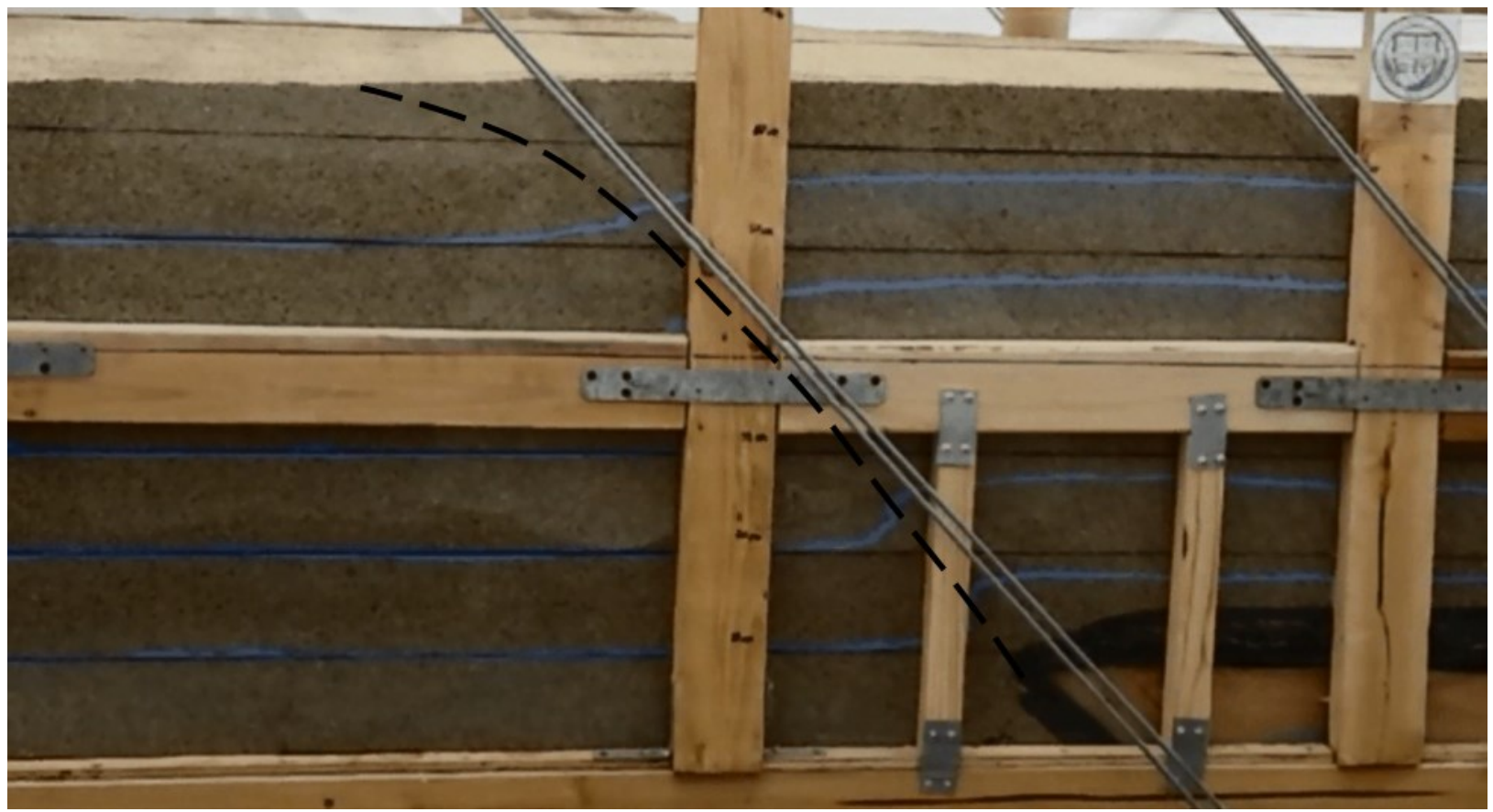

Figure 45: An additional $2.19 \mathrm{~cm}$ of basal displacement beneath the loose sand with a previously existing failure plane. The dotted line represents the rupture plane. The edges have been cropped for clarity. 


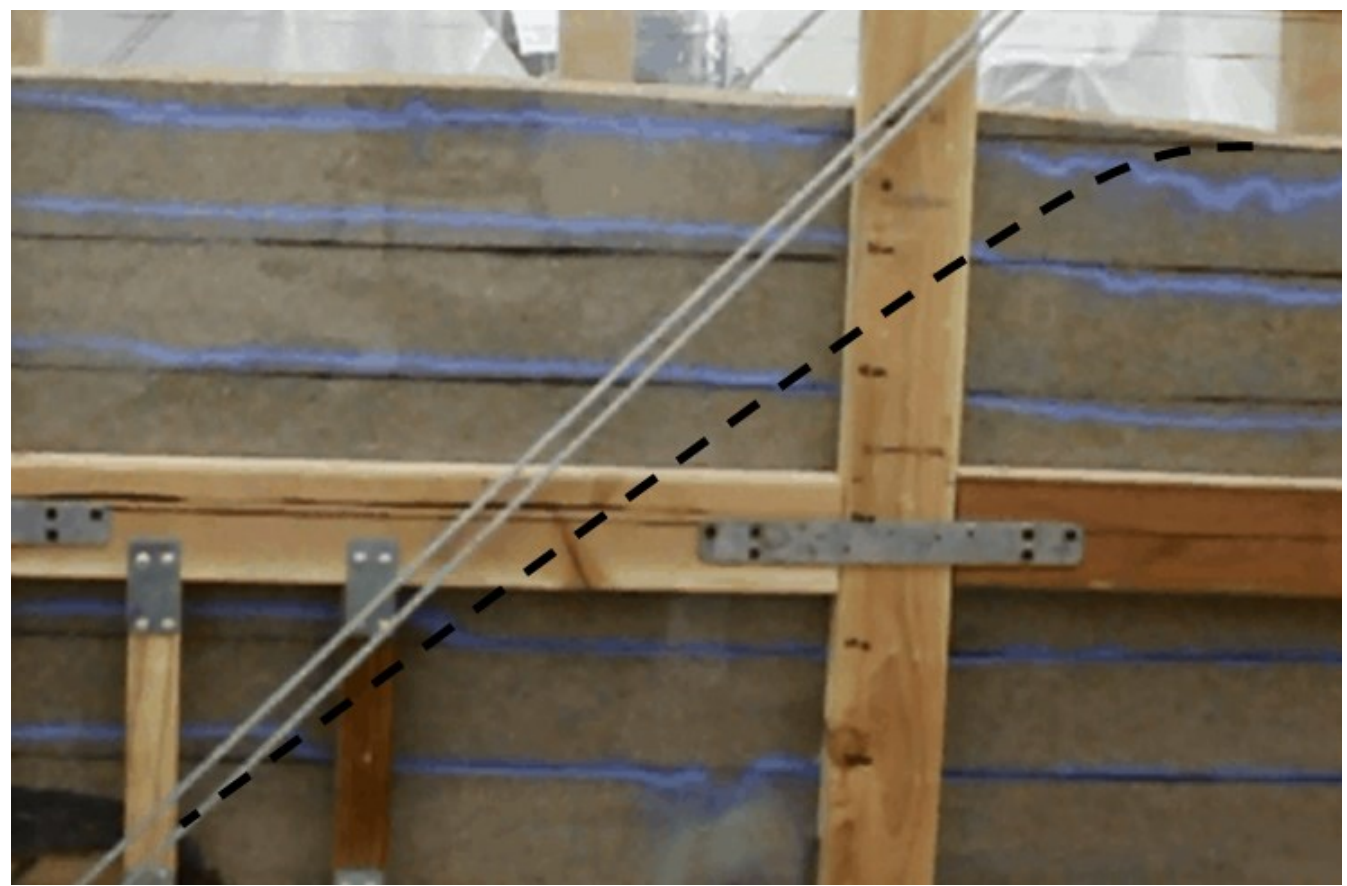

Figure 46: $4.01 \mathrm{~cm}$ of basal displacement beneath virgin dense sand. The dashed line represents the failure plane. The edges have been cropped for clarity.

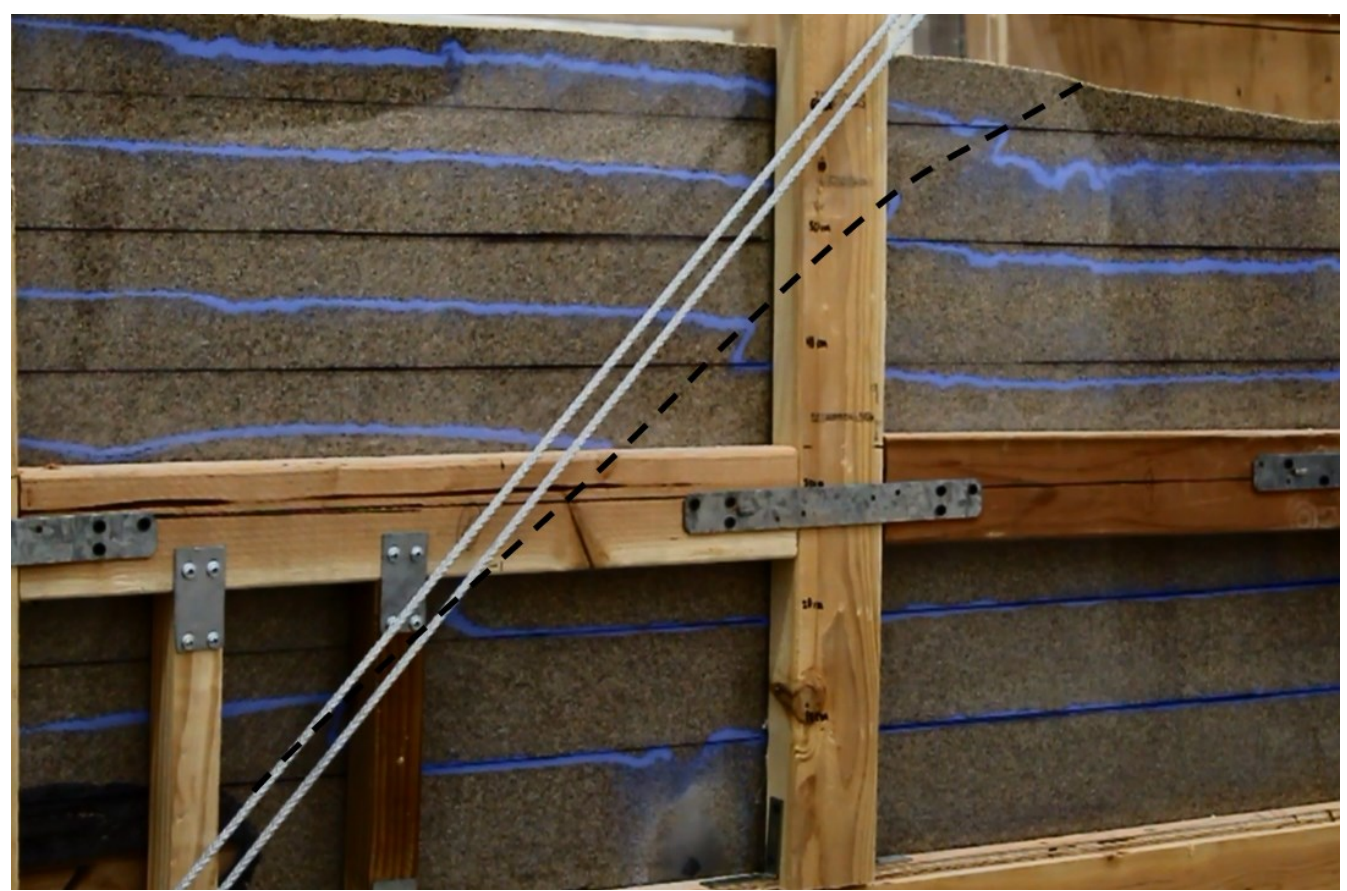

Figure 47: An additional $0.50 \mathrm{~cm}$ of basal displacement beneath disturbed dense sand. The dashed line represents the failure plane. The edges have been cropped for clarity. 


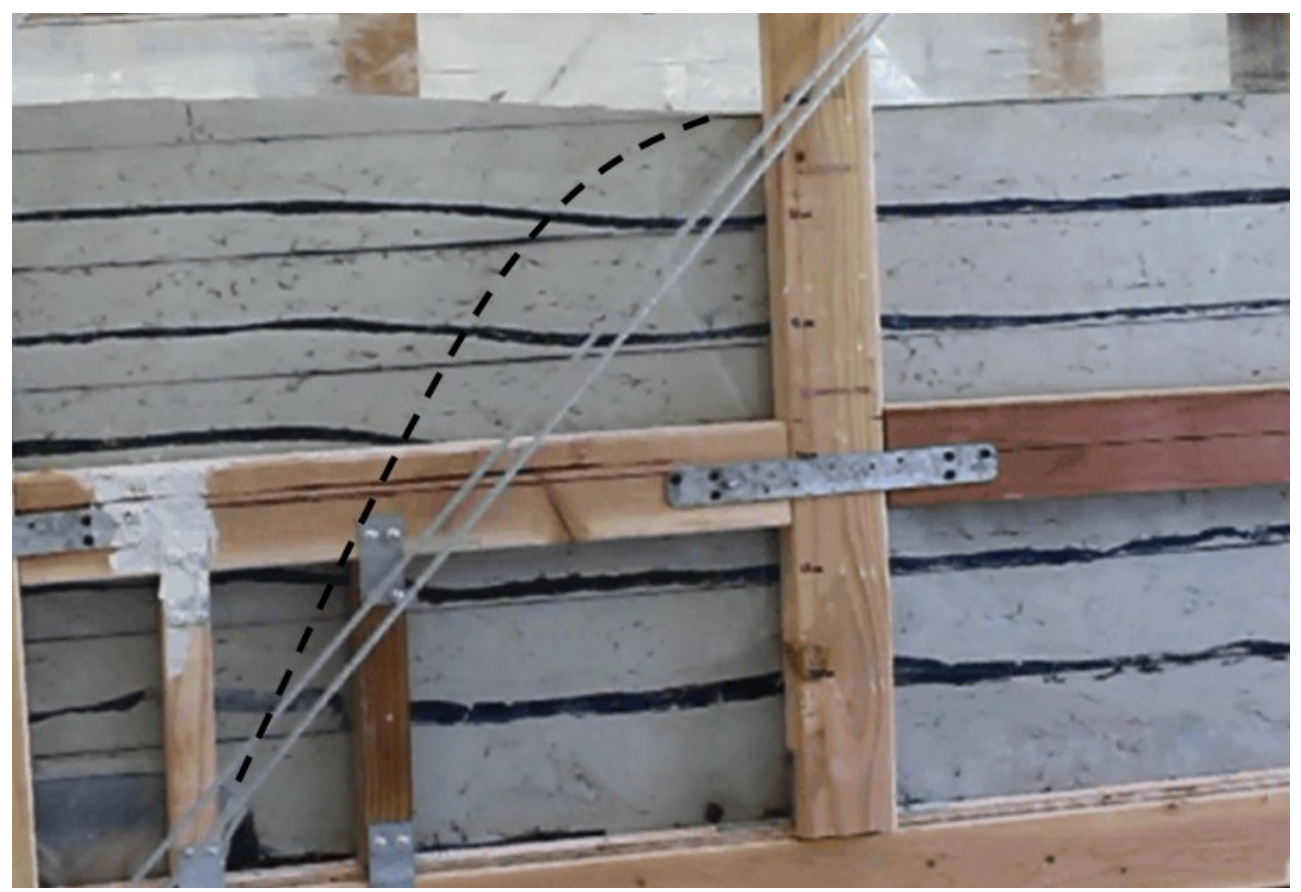

Figure 48: $5.53 \mathrm{~cm}$ of basal displacement beneath virgin stiff clay. The dashed line represents the failure plane. The edges have been cropped for clarity.

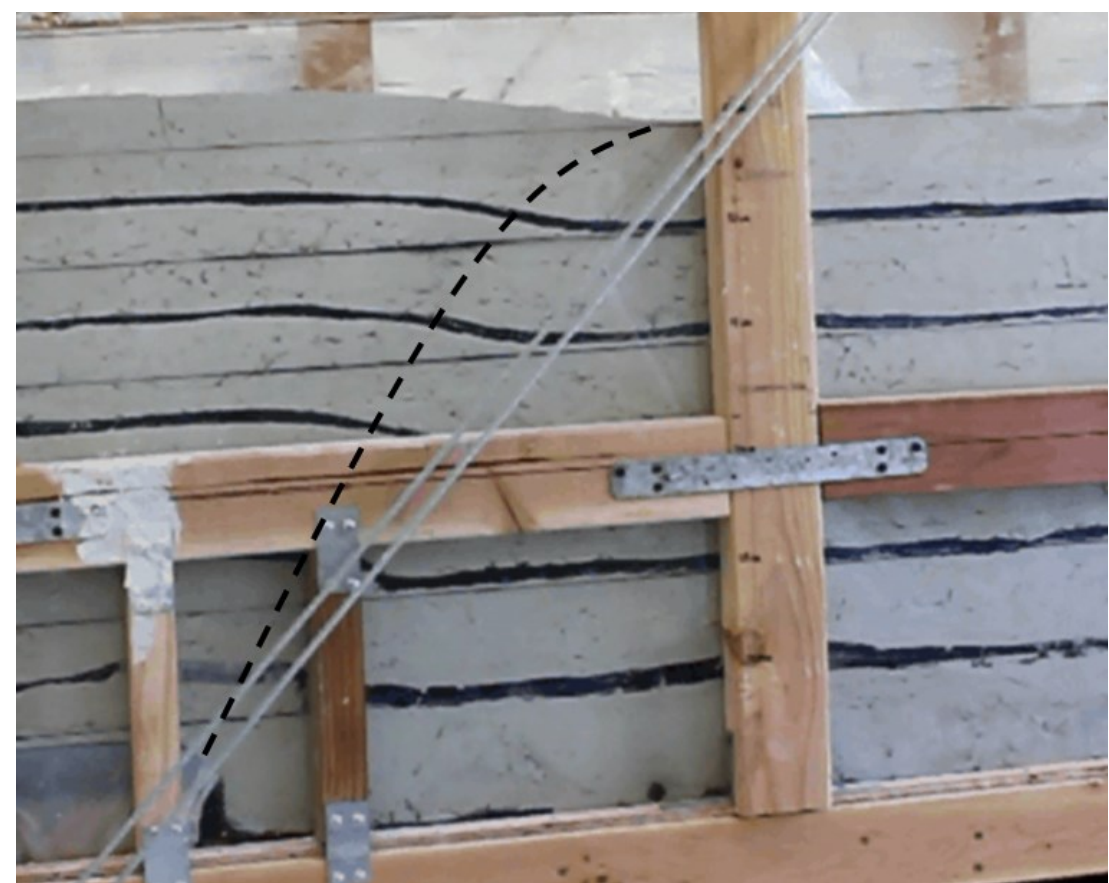

Figure 49: An additional $1.17 \mathrm{~cm}$ basal displacement beneath disturbed stiff clay. The dashed line represents the failure plane. The edges have been cropped for clarity. 


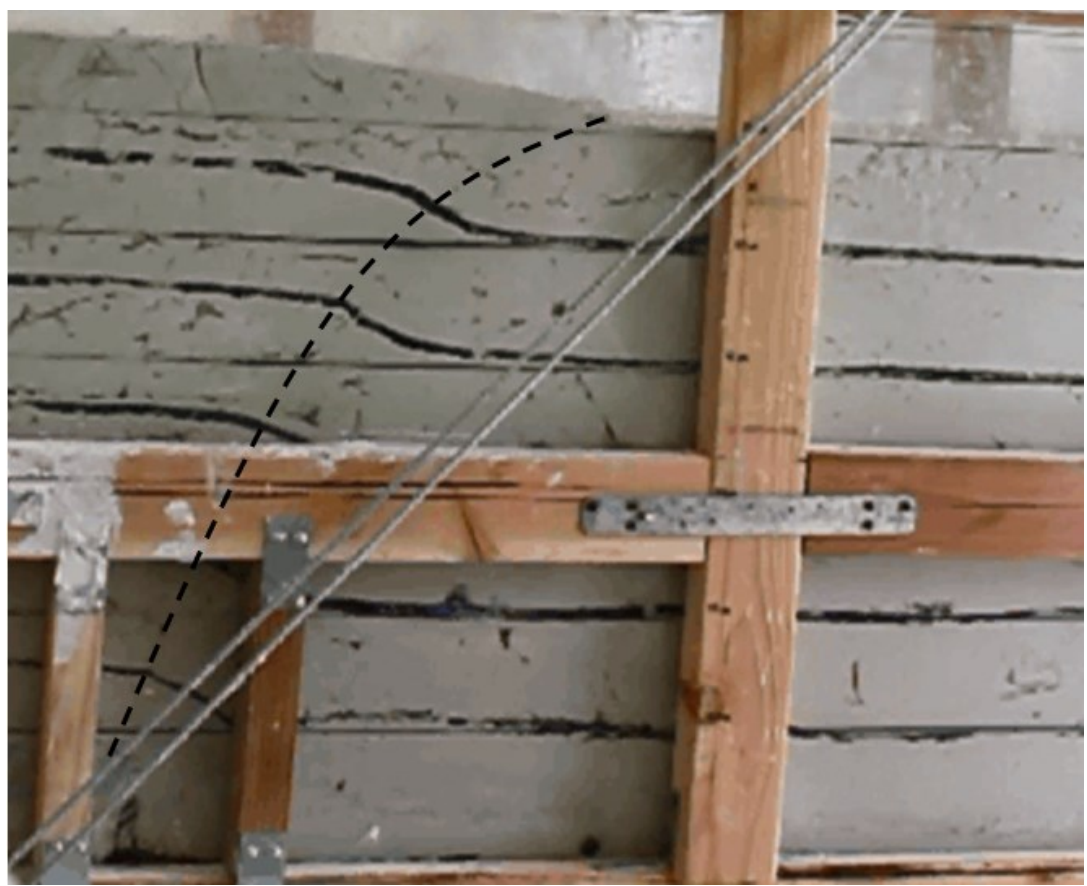

Figure 50: $8.37 \mathrm{~cm}$ of basal displacement beneath virgin soft clay. The dashed line represents the failure plane. The edges have been cropped for clarity.

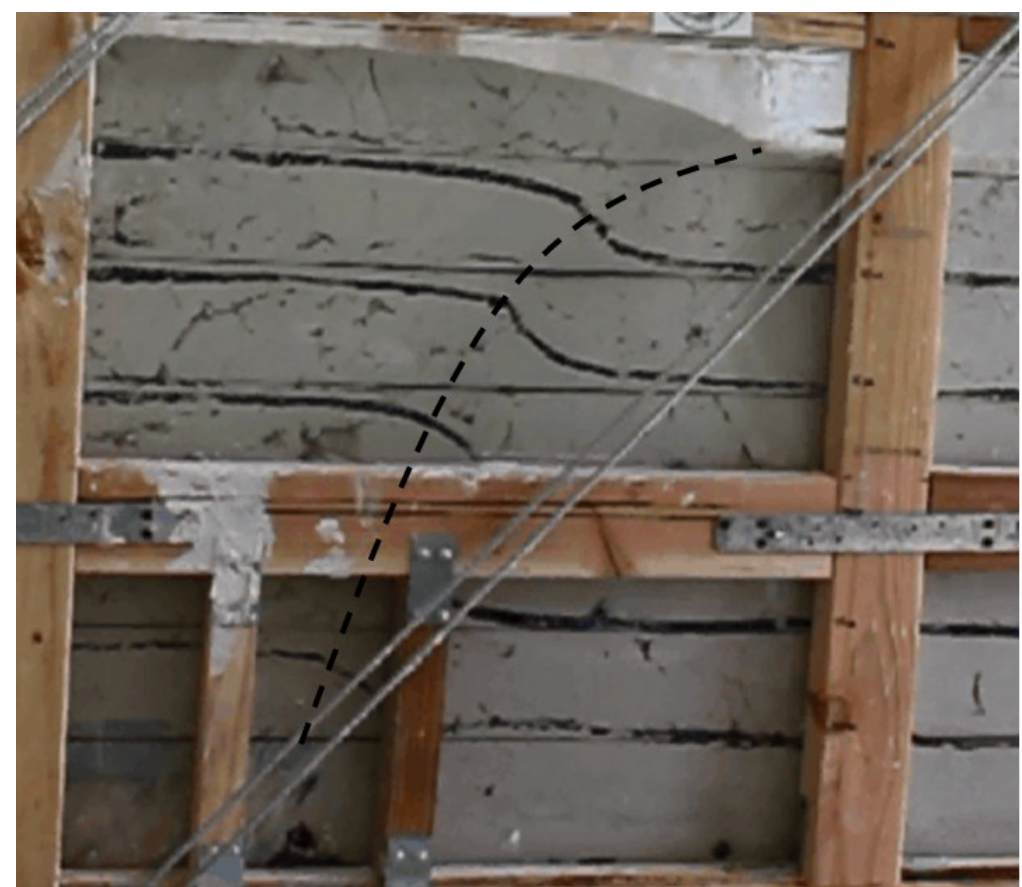

Figure 51: An additional $3.76 \mathrm{~cm}$ of basal displacement beneath disturbed soft clay. The dashed line represents the failure plane. The edges have been cropped for clarity. 


\subsection{Procedure for Determining Displacement at Surface Rupture}

The same two stage rupture process described in Bray (1994b) as well as in Oettle and Bray (2013) was observed in this study. We found that, in general, displacement is first accommodated mainly by broad deformation and then followed by strain localization. However, the initial stage of broad deformation is also accompanied by some level of strain localization. It is when that strain localization reaches the surface that we have entered stage two and surface rupture is defined. For our purpose, accurately recognizing the transition from stage one to stage two was central to obtaining the results given in Chapter 5. Therefore, a repeatable methodology for identifying the failure plane and the point at which it reached the surface was developed and is presented below through an example analysis of the video data collected for virgin dense sand. 
Procedure for determining the basal displacement at surface rupture:

1) Scroll through the video frame-by-frame and identify the point at which the developing rupture plane has passed through the topmost marker bed but has not yet reached the surface. Sketch the corresponding rupture plane at this point in the rupture process (Figure 52).

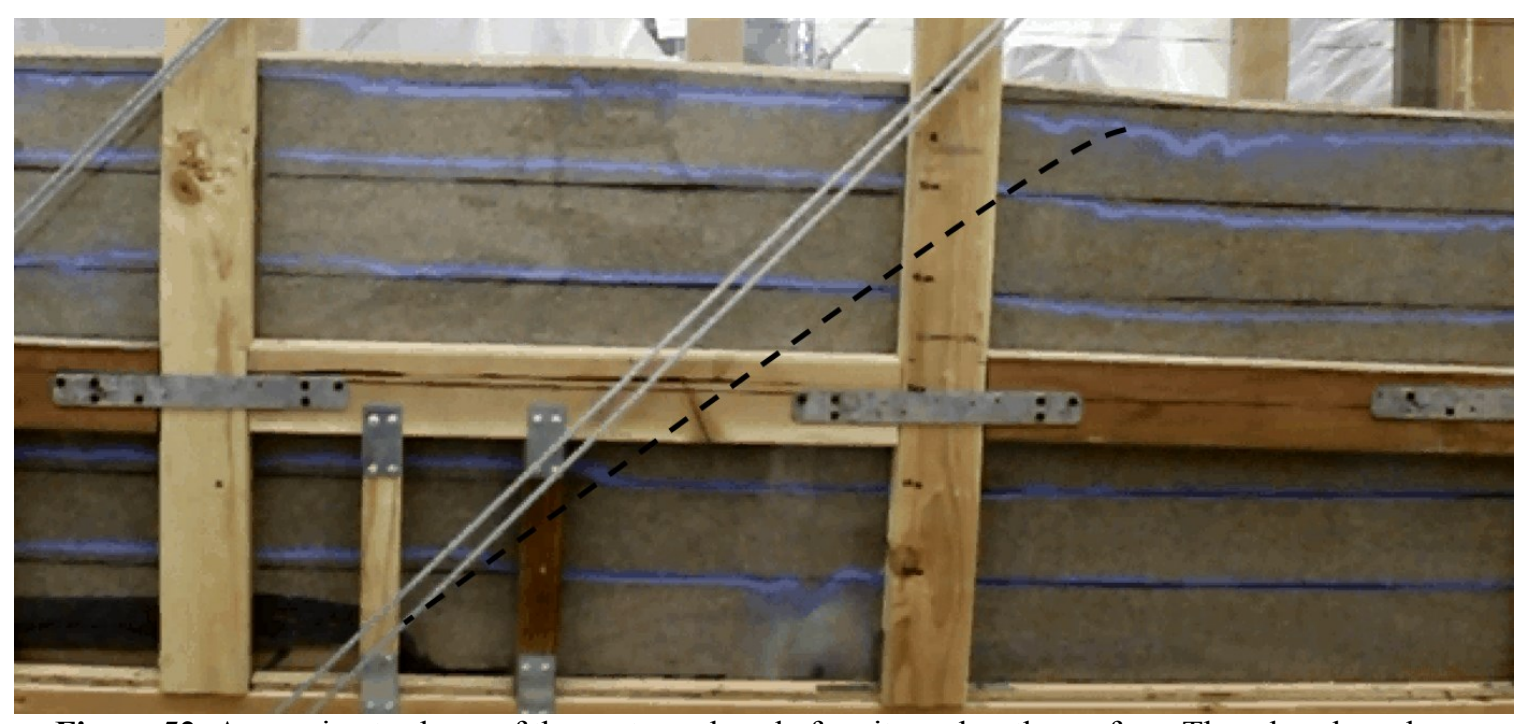

Figure 52: Approximate shape of the rupture plane before it reaches the surface. The edges have been cropped for clarity. 
2) Based on the sketch in step 2, predict the bounds of a zone where surface rupture is likely to occur. Then, with a very fine line, trace the surface expression across this zone for the current frame (Figure 53 and Figure 54). It is helpful to use the color contrast between the material touching the Plexiglass wall and that which is not to accurately define the trace.

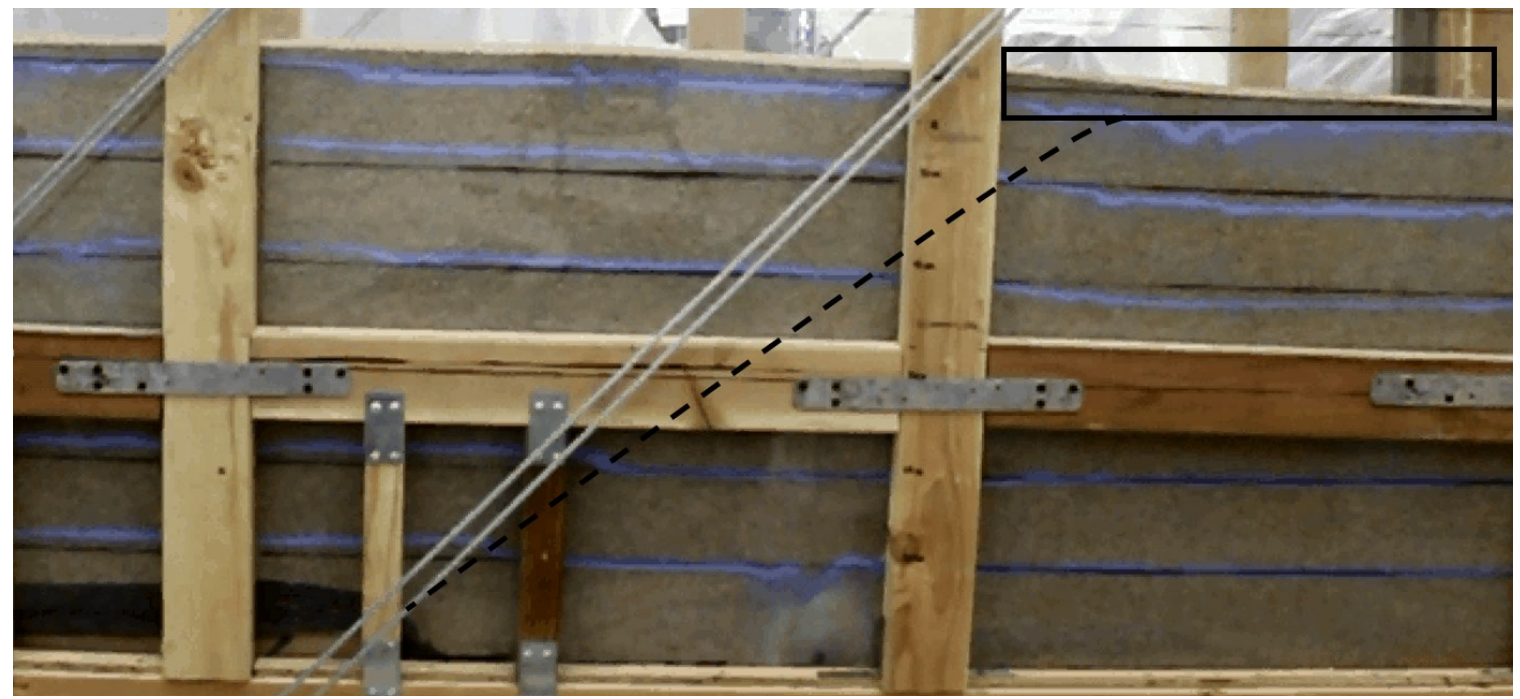

Figure 53: The black box is an estimate of where the rupture plane will reach the surface. The edges have been cropped for clarity.

Figure 54: Close-up view of the projected region from Figure 53 where surface rupture is likely to occur. The black line is a trance of where the sand surface and the nearest Plexiglass wall meet. 
3) Progress through the frames, tracing the surface expression for each until a break in the "smooth line" is perceivable. This is illustrated in Figure 55. For tests on previously ruptured material this process is the same since the scarp is smoothed over between tests.

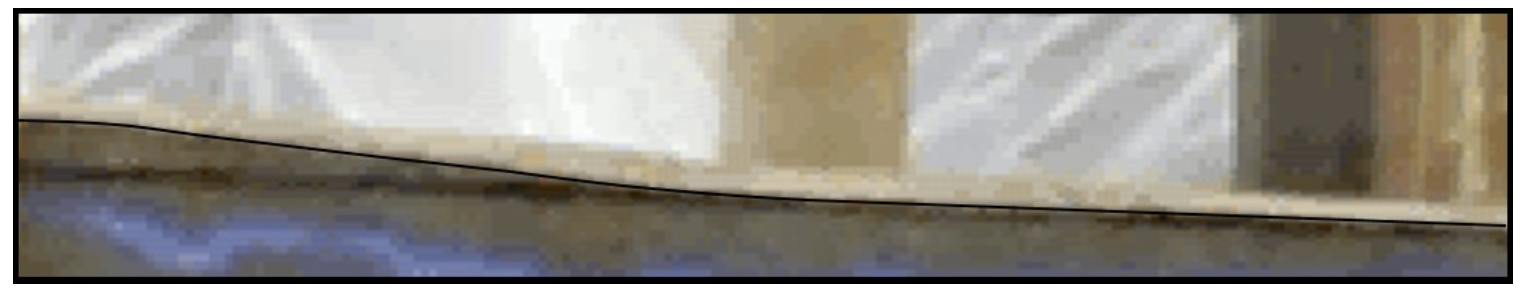

(a)

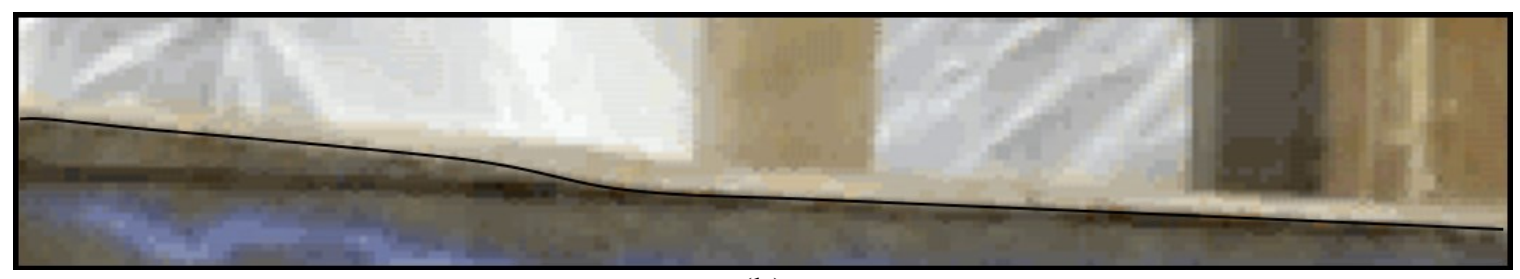

(b)

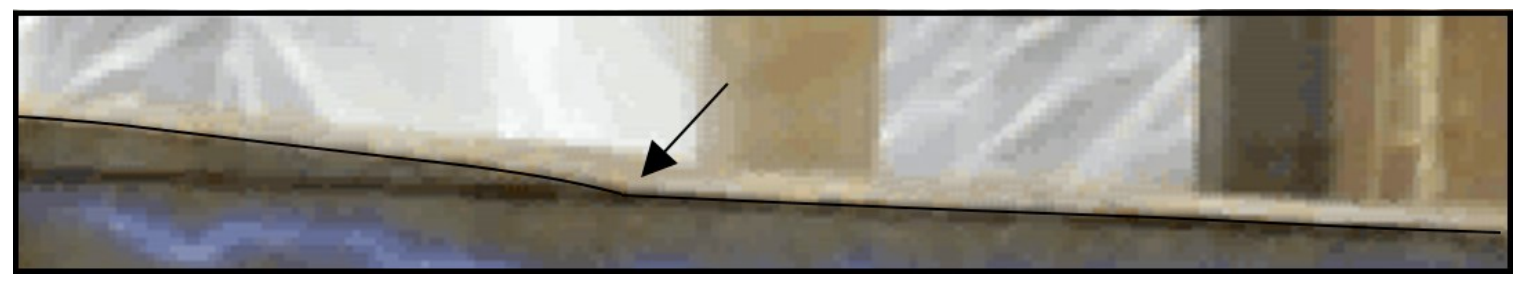

(c)

Figure 55: Examples of three successive video frames leading to surface rupture. (a) no surface rupture; (b) no surface rupture; (c) surface rupture indicated by the "kink" at which the arrow is pointing 
4) Repeat step 2 for the frame in which the first evidence of surface rupture was found to approximate the final shape of the developed rupture plane (Figure 56).

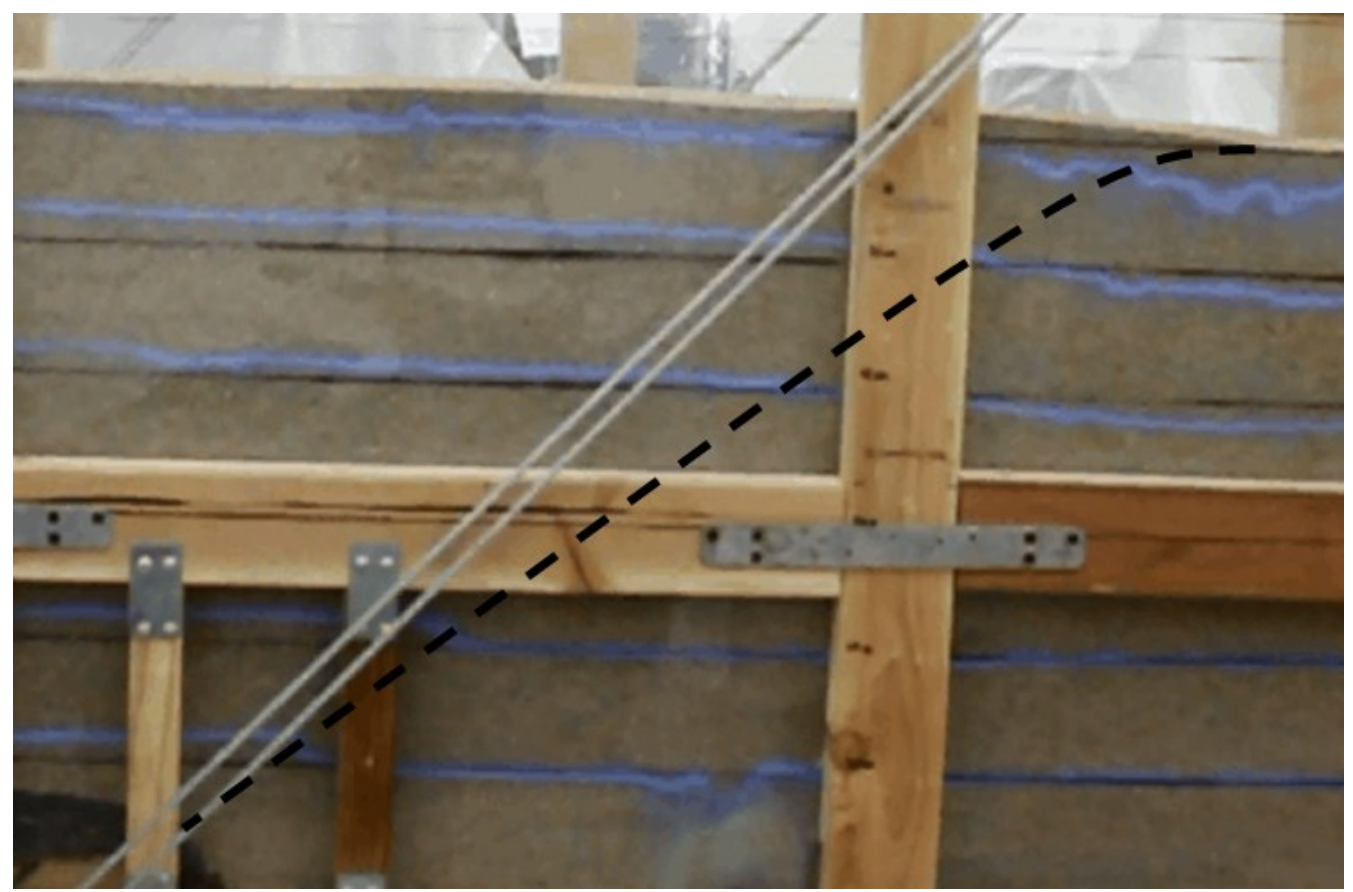

Figure 56: Example of the shape of the rupture plane at the moment surface rupture occurred in virgin dense sand.

Note that this procedure was used in conjunction with inspection of the videos in real time. Two different camera angles were also used to independently arrive at the essentially the same conclusions regarding the basal displacement at surface rupture in all cases. Examples of the two different camera orientations are shown in Figure 57 and Figure 58. 


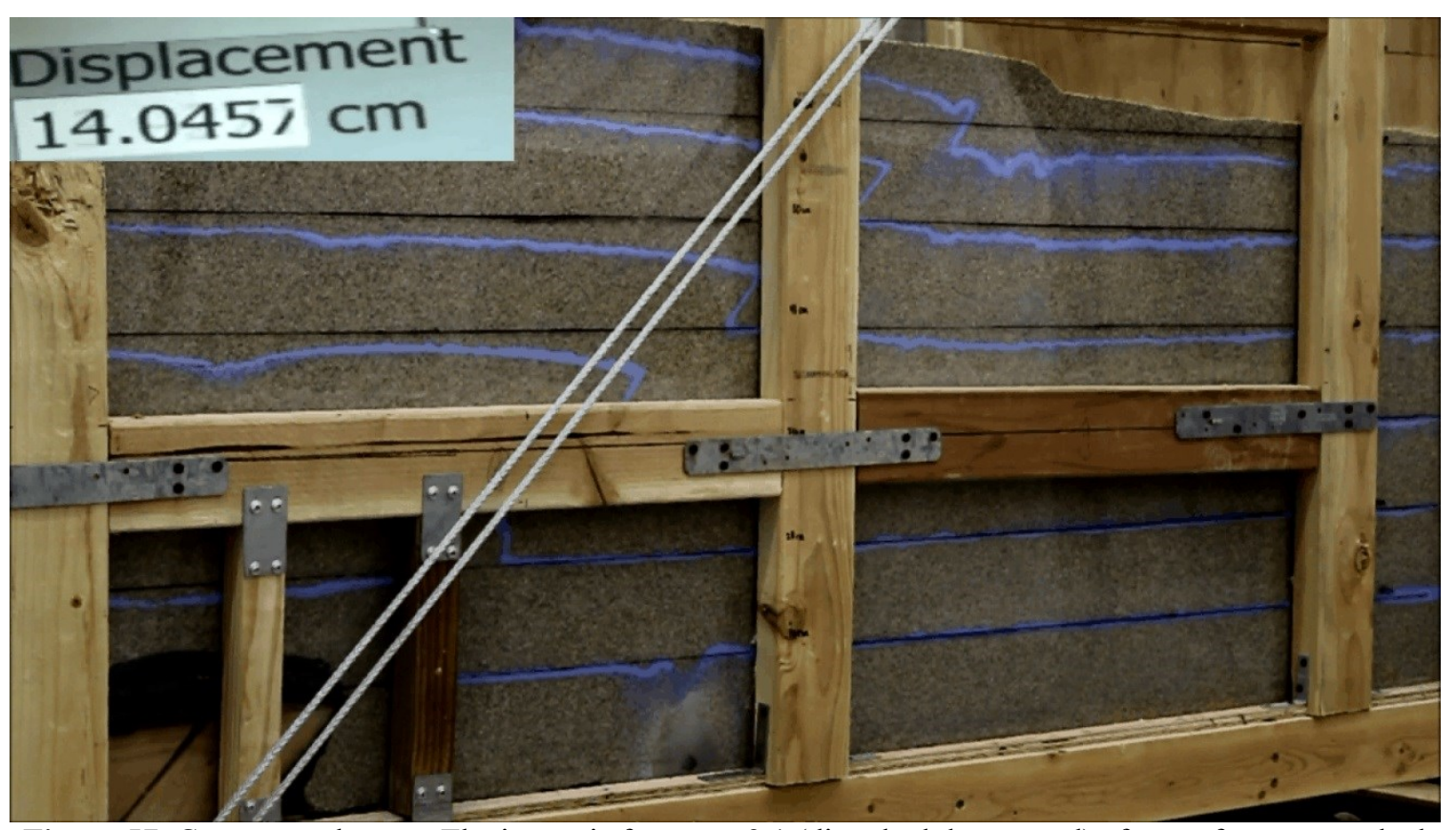

Figure 57: Camera angle one.. The image is from test 2.1 (disturbed dense sand) after surface rupture had occurred.

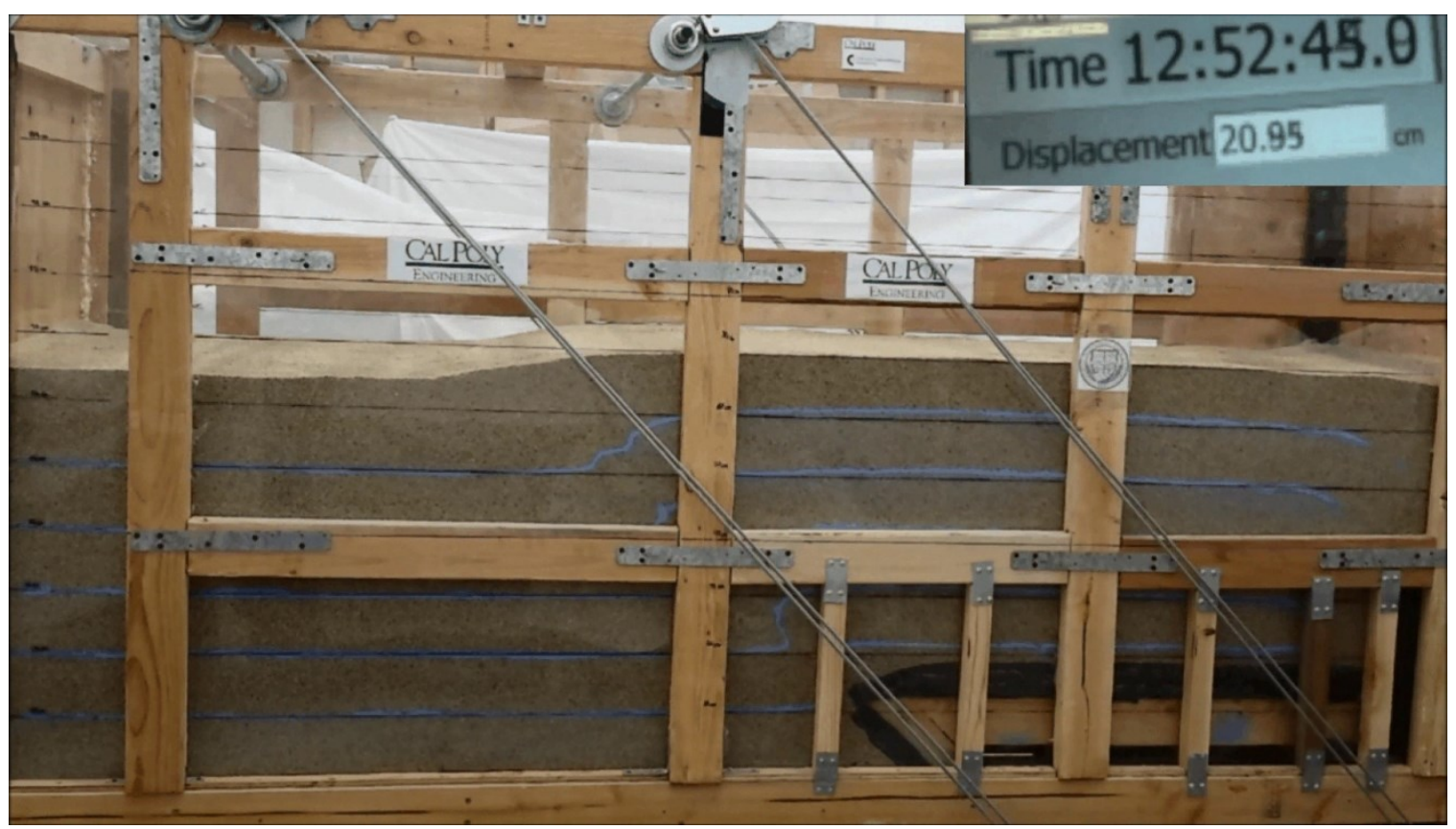

Figure 58: Camera angle two.. The image is from test 1.1 (disturbed loose sand) after surface rupture had occurred. 


\section{Summary and Conclusions}

\subsection{Summary of Findings}

The main takeaway from this study is that material stiffness, material type and rupture history all have an influence on the overall potential for surface rupture in reverse fault conditions. A measure of this is indicated by the value of the normalized basal displacement, $h / H$. Therefore, evaluating the average normalized basal displacement for materials of the same type, relative stiffness and rupture history reveals how each parameter influences the potential for surface rupture. In addition, it also provides some insight as to the relative influence of each parameter, although more data would be needed to properly quantify the relationships.

The results support the initial hypothesis that increased material stiffness would correspond to surface rupture occurring more readily. This is indicated by the observed $132 \%$ increase in the normalized basal displacement required to generate surface rupture when loose/soft material was tested as opposed to dense/stiff. Similarly, the normalized basal displacement for sand was on average $40 \%$ greater than that for clay while the average for virgin materials was 304\% greater than it was for previously ruptured materials. Altogether these findings highlight the importance of considering rupture history and material type in addition to near surface material stiffness when attempting to determine the probability of surface rupture.

It should be noted that the results for material type were not obtained for materials at the same shear wave velocity and therefore do not represent a true measure of the influence of the parameter on the potential for surface rupture. Nevertheless, the fact that the 
average shear wave velocity of clay was lower than sand may be somewhat informative considering the positive correlation between material stiffness and the susceptibility to surface rupture. This suggests that the $40 \%$ difference in the average normalized basal displacement observed herein may underestimate the difference if the materials had been tested at the same shear wave velocity. The results from this study, however, do not provide any conclusive evidence of the influence of material stiffness.

\subsection{Comparisons with Past Research}

The results presented in Chapter 5 concerning material stiffness agree with the findings of Moss et al. (2013) which predicted an increase in the probability of surface rupture given an increase in near surface material stiffness for reverse faults. In addition, Moss et al. (2013) also provides insight as to how important considering rupture history could be for estimating the probability of surface rupture. According to the case-study statistics, the probability of surface rupture when dense material is present in the upper 30 meters is up to $30 \%$ greater than for soft material. Thus, given that this study suggests rupture history plays a greater role than material stiffness, the difference in the probability of surface rupture should be similarly greater if rupture history were used as a predictor variable. However, to accomplish this, a methodology for identifying prior rupture or lack thereof would first need to be developed and even then it may be difficult to achieve statistical significance due to a shortage of cases in which surface rupture occurred for the first time.

For material type, a case-study based analysis might not be practical. Aside from the aforementioned limited information about the relative influence of the parameter, the 
idealized soils which were tested do not exist in nature. This would make it difficult to obtain meaningful data for a case-study based analysis very difficult.

Anastosopoulos et al. (2007) predicted that increasing material stiffness would have a qualitatively consistent effect for both sand and clay. This is another prediction which the results herein support. Although we observed a difference in the overall potential for cohesive and cohesionless materials, both required greater basal displacement to initiate surface rupture when material stiffness was increased. Despite this agreement, however, the normalized basal displacement required for surface rupture based on the Anastosopoulos et al. (2007) finite element model was much smaller for loose sand than that of this study. Unfortunately, there are no other precise quantitative results to compare against for loose sand since Cole and Lade (1984) did not quantify this value and no other studies distinguish between loose and dense. The most appropriate parallel from other research is from the centrifuge tests conducted by Bransby et al. (2008) on sand. They reported a value for the normalized basal displacement which roughly agrees with the findings from this study, $h / H=0.1$, and is significantly greater than that estimated by Anastosopoulos et al. (2007) for loose sand, $h / H=0.04$. The difference may be attributed to other properties of the loose sand not capture by the "loose" description, which is in no way a quantitative measure of material stiffness. Parameters such as gradation or mean particle size affect the overall material stiffness whether or not the sand has been densified. This highlights an inherent difficulty in comparing the results of this study to those of previous work. Since not all prior fault rupture studies were concerned with material stiffness, meaningful quantitative comparisons cannot be made. 
To gauge the quality of the results it is useful to compare the values of normalized basal displacement to that of other published work wherever possible. Aside from what has already been discussed, other past results are very similar to those herein.. The 1-g fault box studies conducted by Cole and Lade (1984), Taniyama and Watanabe (2001), and Tani (1994) all provide data which agrees very closely with that of this study for dense sand. In addition,Bray et al. (1994b) used finite element procedures to predict similar values for normalized basal displacement for saturated clay. Table 9 and Figure 59 provide a summary of existing comparable evidence.

Table 9: Normalized basal displacement required for surface rupture from this study and other comparable research.

\begin{tabular}{|c|c|c|c|}
\hline Soil Type & $\begin{array}{l}\text { Experiment } \\
\text { Description }\end{array}$ & $\begin{array}{c}(h / H) \text { at Surface } \\
\text { Rupture }\end{array}$ & Reference \\
\hline Loose Sand & 1-g Fault Box, $45^{\circ}$ dip & 0.147 & this study \\
\hline Dense Sand & 1-g Fault Box, $45^{\circ}$ dip & 0.047 & this study \\
\hline Soft Clay & 1-g Fault Box, $45^{\circ}$ dip & 0.097 & this study \\
\hline Stiff Clay & 1-g Fault Box, $45^{\circ}$ dip & 0.014 & this study \\
\hline Saturated Clay & Finite Element, $45^{\circ}$ dip & $0.039-0.056$ & Bray et al. (1994b) \\
\hline Dense Sand & Finite Element, $45^{\circ}$ dip & 0.025 & Anastasopoulos et al. (2007) \\
\hline Loose Sand & Finite Element, $45^{\circ}$ dip & 0.040 & Anastasopoulos et al. (2007) \\
\hline Sand & Centrifuge, $60^{\circ}$ dip & 0.10 & Bransby et al. (2008) \\
\hline Dense Sand & Fault Box, 1-g & $0.022-0.044$ & Cole and Lade (1984) \\
\hline Loose Sand & Fault Box, 1-g & $>0.044 *$ & Cole and Lade (1984) \\
\hline Dense Sand & 1-g Fault Box, $45^{\circ}$ dip & $0.04-0.05$ & Taniyama and Watanabe (2001) \\
\hline Dense Sand & 1-g Fault Box, $45^{\circ}$ dip & $0.03-0.06$ & Tani (1994) \\
\hline
\end{tabular}

${ }^{*}$ Cole and Lade (1984) states that the basal displacement required to reach surface rupture was significantly greater for loose sand than dense, though no precise value of $h / H$ was given. 


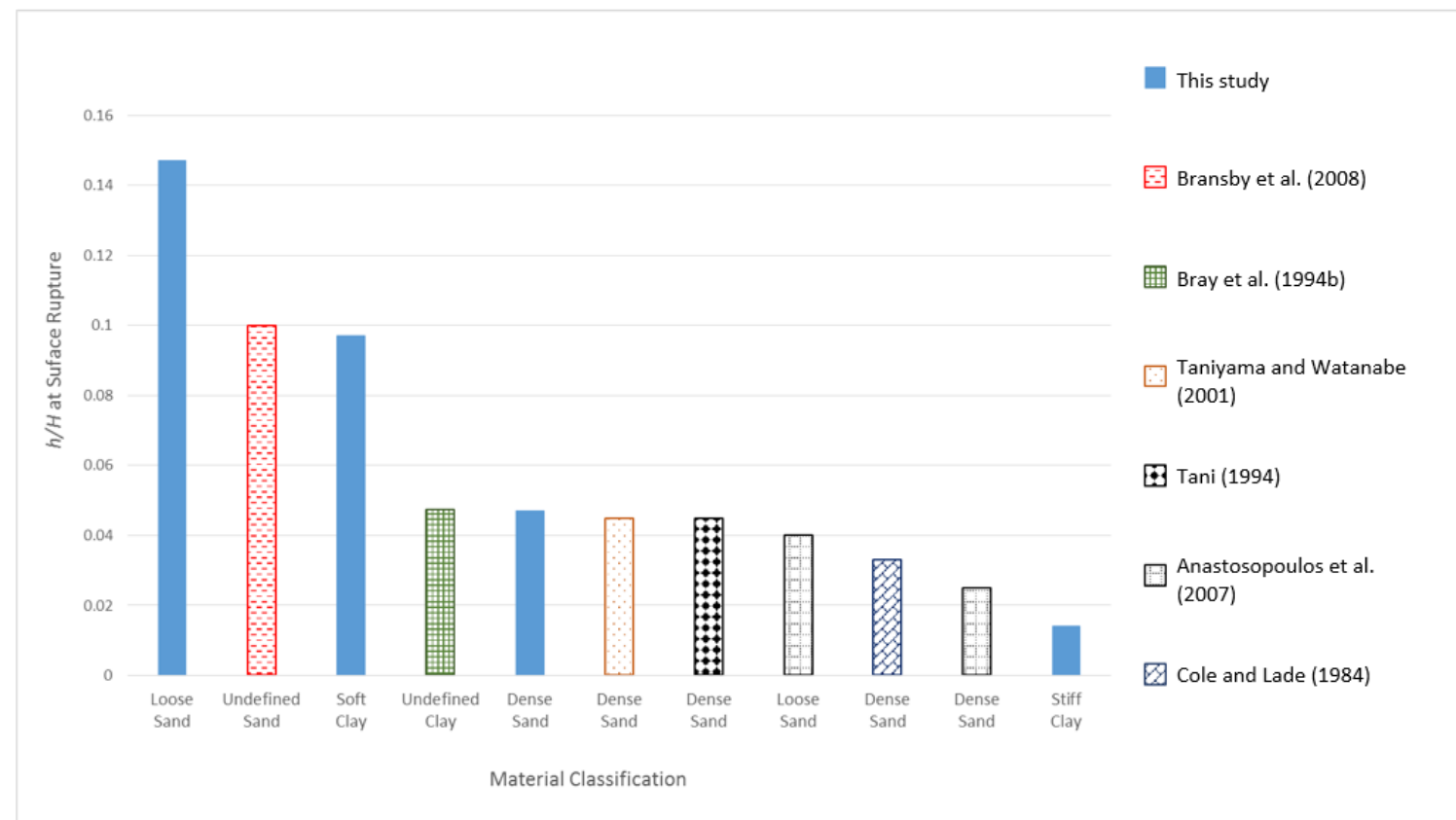

Figure 59: The data from this study compared to other research. "Undefined" indicates relative stiffness was not available.

From left to right, the trend in the data presented in Figure 59 is one of increasing susceptibility to surface fault rupture. In all cases but one, for the same relative stiffness, clay was found to be more prone to surface rupture than sand. Furthermore, all of the data presented supports the notion the increasing material stiffness promotes surface rupture for a given material type. Therefore, other than one outlier, which is from the Anastosopoulos (2007) results for loose sand, all available data from prior research agrees with the general findings of this study.

Since the experiments herein were performed at $1 \mathrm{~g}$, the body forces which tend to govern the engineering properties of course grained materials were not scaled properly. However, comparison between centrifuge experiments performed for other research and the results of this study and of Cole and Lade (1981) provides some insight as to the effects of accurately scaled gravity forces and corresponding effective stresses for tests 
on sand. Surprisingly, the 50g centrifuge tests from Roth et al. (1981) and the $115 \mathrm{~g}$

centrifuge tests from Bransby et al. (2008) both produced strikingly similar results as the fault box tests performed at $1 \mathrm{~g}$ in this study and by Cole and Lade (1984). This suggests that improperly scaled body forces did not significantly affect the rupture process in the tests on sand in this study. If this is true, then the conclusions drawn should be valid at larger scales.

\subsection{Sources of Experimental Error}

\subsubsection{Defining Surface Rupture in Loose Sand}

When localized soil failure first reached the surface in the loose sand tests, there was no visible scarp spanning the width of the fault box. The video data, which recorded the profile view, showed that the shear band reached the soil surface long before it was perceivable from a plan view. Thus, it is important to note that the basal displacement corresponding to surface rupture was taken as the point at which the shear band reached the surface, not the point at which a scarp was visible from above. With this in mind, the reported offset, from Table 8, required for surface rupture in loose sand could be considered somewhat conservative compared that of the other materials. In all other cases, surface rupture was discernible from the soil surface as well as from the profile view at the basal displacements reported in Table 8 . For the loose sand tests, however, surface rupture was only evident from the profile view and was much more difficult to discern than any of the other tests using the procedure presented in the previous chapter. This was because the smooth curves characteristic of broad deformation were only slightly disrupted at the onset of surface rupture. A possible explanation for this could be 
that the angle of repose for the loose sand happened to be similar to that of the slope produced by broad deformation when the shear band breached the surface. This would have cause sand to fall back over the developing scarp, effectively masking the signs of surface rupture. Interestingly, the same effects were observed for the tests in dense sand only after surface rupture had already been defined.

\subsubsection{Disturbance Prior to Test 2.0}

The rupture plane in test 2.0 reached the surface at a different horizontal position on one side of the fault box than the other. The location of surface rupture on the right side of the box was approximately $3 \mathrm{~cm}$ closer to the edge of the front end of the fault box. This may have been caused by a leak that developed before the test was run on the left side of the box, near the nucleation point. Although the leak was quickly stopped, the change in the stress state of the material near the base of the profile in that area seemed to cause the rupture plane to divert around the disturbed sand. Nevertheless, surface rupture was achieved at virtually the same basal displacement for each of the two sides in the tests on dense sand and the shape of the failure plane was roughly same except for the small deviation at the base.

\subsubsection{Effects of Marker Beds}

The Chaulkline marker beds used in the sand tests differed from the elastic strips used for the clay in that the Chaulkline markers actually failed as the rupture plane passed through them while the elastic strips did not. Fortunately, the elastic strips were still effective in highlighting where the rupture was but the information had to be determined based on how the strips deformed, not where they failed. This introduced the potential for an 
improper interpretation and great care had to be taken to identify the portion of each strip which spanned the shear band.

Another issue with the marker beds was that they both were easily distorted prior to testing. For example, before the tests on dense sand, the densification process significantly disturbed some of the marker layers. Additionally, the elastic strips for all of the tests on clay were subject to significant disturbance in some locations during our efforts to pack the clay uniformly against the Plexiglass. Despite this, the distorted marker beds did not prevent the video records from portraying the relative offset on either side of the developing shear band, which is all that was necessary to identify the failure plane.

\subsubsection{Smoothing the Surface after Phase 1}

Leveling or smoothing the soil surface after each test on virgin material slightly changed the overburden stress on either side of the slip plane some amount. However, the effects on rupture propagation were likely negligible since the change in depth never exceeded four centimeters in any given location between any of the tests. Furthermore, inspection of the video records clearly revealed that rupture through disturbed material occurred on the same plane as the corresponding virgin test in all cases.

\subsubsection{Effects of Wall Friction}

In the surface expression from each test conducted, the fault scarp emerged slightly closer to the front end of the fault box near the center of its width than it did near the Plexiglass walls. This was likely due to the influence of wall friction but other factors may have also contributed such as spatial variations in density or the presence of the marker beds. 
Regardless of the source of the effect, the deviations from uniform surface rupture across the width of the fault box were minor. In addition, the video records revealed that surface rupture occurred at essentially the same time (i.e. same basal displacement) at all locations. Thus, wall friction may have slightly altered the shape of the rupture planes but has had a negligible impact on the basal displacement required for surface rupture.

The horizontal distance between the scarp emergence locations at the center and edges of the soil surface was estimated after phase 1 of each test. Following, the percent error was calculated assuming that the true rupture path occurred at the center of the fault box where the distance between rupture initiation and emergence at the surface was the greatest. This information is presented in Table 10 and indicates that wall friction did not significantly alter the rupture path.

Table 10: Summary of the deviations in fault emergence locations for phase 1 of each test.

\begin{tabular}{|c|c|c|c|}
\hline \multirow{2}{*}{ Material } & \multicolumn{2}{|c|}{$\begin{array}{c}\text { Horizontal Distance Between Rupture } \\
\text { Initiation and Surface Rupture (cm) }\end{array}$} & $\begin{array}{c}\text { Percent Error } \\
\text { (\%) }\end{array}$ \\
\cline { 2 - 3 } & Maximum & Minimum & \\
\hline Loose Sand & 73 & 72 & 1.4 \\
\hline Dense Sand & 90 & 85 & 5.6 \\
\hline Stiff Clay & 52.5 & 50 & 4.8 \\
\hline Soft Clay & 43 & 42 & 2.3 \\
\hline
\end{tabular}

\subsection{Recommendations for Future Research}

More data is needed to develop a complete understanding of relative influence of each parameter on the potential for surface fault rupture. The shear wave velocity of each material was different for all cases in this study which makes drawing conclusions about the impact of material type difficult. To improve on this, comparisons would need to be made based on different materials which share the same shear wave velocity. It is also 
possible that the trends given in Figure 43 are actually nonlinear. Thus, tests would need to be conducted over a range of shear wave velocities to fully quantify the relationships.

Considering that past research has suggested that the effect of scaled effective stresses on rupture propagation in sand is minimal, additional investigations should be conducted to determine the precise influence of $1 \mathrm{~g}$ versus centrifuge style fault box tests. If there really is a negligible difference, then research should be carried out to evaluate what other factors may have caused the differences seen between sand and clay herein.

It would also be meaningful to conduct a study analogous to this but for a strike-slip mechanism. This could validate, or potentially invalidate, the findings of Moss et al. (2013) for strike-slip faulting which predicts no change in the probability of surface rupture with varying material stiffness. Either outcome would be hugely important to improve our ability to predict the chances of surface rupture in any geologic setting. Also, such a study could test if the trend holds true for other parameters such as material type and rupture history.

All physical experiments could serve as valuable tools for calibration in numerical based research. It is recommended that finite element models be developed to simulate fault rupture problems and be used to perform much more robust studies to identify additional potentially impactful parameters on the probability of surface rupture. Furthermore, properly calibrated finite element procedures or even suites of physical experiments could be useful for determining how surface rupture potential is affected for a range of material stiffness contrasts for a given material type. This would allow conclusions to be drawn that would not require weighting the normalized basal displacement by the shear 
wave velocity. Instead, for a given material type, they would identify the complete relationship between normalized basal displacement and material stiffness, which could be nonlinear.

Whenever possible, case study-based statistics should be used to quantify the impact of any parameter for which evidence is found suggesting it is significant in fault rupture processes. Hence, based on the results of this study, it would be appropriate to correlate near surface material type with the probability of surface rupture. Although rupture history should not be neglected in future research, it is assumed that most surface rupture incidents in nature are repeat events. Therefore, an involved investigation concerning its influence is not warranted. Only parameters which display measurable variation across different geologic settings are important for probabilistic frameworks such as PFDHA.

For future studies involving relatively large scale fault box experiments such as this, it is recommended to place an extra emphasis on designing an apparatus which is easily loaded and unloaded. This may accomplished using walls which are removable or could be incrementally built up as soil is loaded into the box. Some of the most difficult and time consuming aspects concerning the experimentation in this study were loading, unloading and placing marker beds in a manner that did not disturb virgin materials. It is also advised to conduct a series of preliminary tests with granular material to ensure that repeated operation is possible without material escaping the box. 


\section{REFERENCES}

Anastosopoulos, I., Gazetas, G. M., Bransby, M., Davies, M., \& El Nahas, A. (2007). Fault Rupture Propagation through Sand: Finite-Element Analysis and Validation through Centrifuge Experiments. Journal of Geotechnical and Geoenvironmental Engineering, 133(8), 943-958.

Bransby, M. F., Davies, M. R., El Nahas, A., \& Nagaoka, S. (2008). Centrifuge modeling of reverse fault-foundation interaction. Bulletin of Earthquake Engineering, 6(4), 607-628.

Bransby, P. L., \& James, R. G. (1970). Experimental and Theoretical Investigations of a Passive Earth Pressure Problem. Geotechnique, 20(1), 17-37.

Bransby, P. L., \& Smith, A. A. (1975). Side Friction in Model Retaining Wall Experiments. Journal of the Geotechnical Engineering Division, 615-632.

Bray, J. D., Seed, R. B., \& Seed, H. (1994b). Analysis of Earthquake Fault Rupture Propagation Through Cohesive Soil. Journal of Geotechnical Engineering, $120(3), 562-580$.

Bray, J. D., Seed, R. B., Cluff, L. S., \& Seed, H. (1994a). Earthquake Fault Rupture Propagation Through Soil. journal of Geotechnical Engineering, 120(3), 543-561.

Cole, D. A., \& Lade, P. V. (1984). Influence Zones in Alluvium Over Dip-Slip Faults. Journal of Geotechnical Engineering, 110(5), 599-615. 
Crosariol, V. A. (2010). Scale Model Shake Table Testing of Underground Structures in Soft Clay. Thesis, California Poytechnic State University.

Holtz, R. D., \& Kovacs, W. D. (1981). An Introduction to Geotechnical Engineering. Prentice-Hall.

James, R. G., \& Bransby, P. L. (1971). A velocity Field for some Passive Earth Pressure Problems. Geotechnique, 21(1), 61-83.

Jayasree, P., Rajagopal, K., \& Gnanendran, C. (2012). Influence of Sidewall Friction on the Results of Small-Scale Labratory Model Tests: Numerical Assessment. International Journal of Geomechanics, 12(2), 119-126.

Johansson, J., \& Konagai, K. (2004). Fault Induced Permanent Ground Deformations Simulations and Experimental Verification. 13th World Conference on earthquake Engineering. Vancouver, B.C., Canada.

Lettis, W. R., Wells, D. L., \& Baldwin, J. N. (1997). Empirical Observations Regarding Reverse Earthquakes, Blind Thrust Faults, and Quaternary Deformation: Are Blind Thrust Faults Truly Blind? Bulletin of the Seismological Society of America, 87(5), 1171-1198.

Meymand, P. (1998). Shaking table scale model tests of nonlinear soil-pile superstructure interaction in soft clay. Ph.D. Disseration, University of California, Berkeley.

Moss, R. E., \& Ross, Z. E. (2011). Probabilistic Fault Displacement Hazard Analysis. Bulletin of the Seismological Society of America, 101(4), 1542-1553. 
Moss, R. E., Crosariol, V., \& Kuo, S. (2010). Shake Table Testing to Quantify Seismic Soil-Structure Interaction of Underground Structures. Recent Advances in Geotechnical Earthquake Engineering and Soil Dynamics. San Diego, California.

Moss, R. E., Stanton, K. V., \& Buelna, M. I. (2013). The Impact of Material Stiffness on the Likelihood of Fault Rupture Propagating to the Ground Surface. Seismological Research Letters, 84(3), 485-488.

Oettle, N. K., \& Bray, J. D. (2013). Fault Rupture Propagation Through Previously Ruptured Soil. Bulletin of the Seismological Society of America.

Petersen, M., \& others. (2011). Fault Displacement Hazard for Strike-Slip faults. 101(2), 805-825.

Roth, W., Scott, R., \& Austin, I. (1981). Centrifuge Modeling of Fault Propagation Through Alluvial Soils. Geophysical Research Letters, 8(6), 561-564.

Stepp, J. C., Wong, I., Whitney, J., Quittmeyer, R., Abrahamson, N., Toro, G., . . . Y.M.P.P. Members. (2001). Probabilistic Seismic Hazard Analysis for Ground Motions and Fault Displacements at Yucca Mountain, Nevada. Earthquake Spectra, 17(1), 113-151.

Tani, K. (1994). Numerical Simulation of Model Test of Reverse Fault by FEM Incorporating Joint Elements. Proceedings of Symposium on Subsurface Failure and Localization of Strain, (pp. 215-222).

Taniyama, H., \& Watanabe, H. (2001). Deformation of Sandy Deposits by Reverse Faulting. Seismic Fault-induced Failures, (pp. 135-142). Tokyo. 
Wells, D. L., \& Coppersmith, K. J. (1994). New Empirical Relationships among Magnitude, Rupture Length, Rupture Width, Rupture Area, and Surface Displacement. Bulletin of the Seismological Society of America, 84(4), 974-1002.

Wroth, C. P., \& Bassett, R. H. (1965). A Stress-Strain Relationship for the Shearing Behaviour of a Sand. Geotechnique, 15(1), 32-56.

Youngs, R., Arabasz, W. J., Anderson, R. E., Ramelli, A. R., Ake, J. P., Slemmons, D. B., . . Toro, G. R. (2003). A Methodology for Probabilistic Fault Displacement Hazard Analysis (PFDHA). Earthquake Spectra, 19(1), 191-219. 